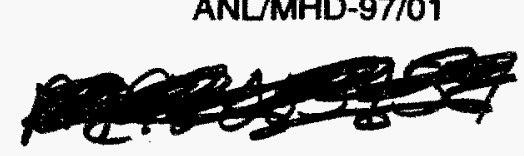

\title{
Multiphase Integral Reacting Flow Computer Code (ICOMFLO): User's Guide
}

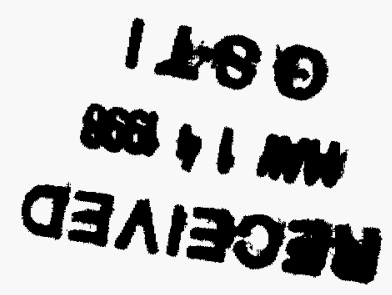

Energy Systems Division Argonne National Laboratory

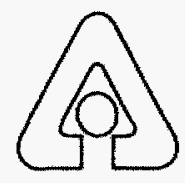

Operated by The University of Chicago, under Contract W-31-109-Eng-38, for the

United States Department of Energy 
Argonne National Laboratory, with facilities in the states of Illinois and Idaho, is owned by the United States government, and operated by The University of Chicago under the provisions of a contract with the Department of Energy.

\section{DISCLAIMER}

This report was prepared as an account of work sponsored by an agency of the United States Government. Neither the United States Government nor any agency thereof, nor any of their employees, makes any warranty, express or implied, or assumes any legal liability or responsibility for the accuracy, completeness, or usefulness of any information, apparatus, product, or process disclosed, or represents that its use would not infringe privately owned rights. Reference herein to any specific commercial product, process, or service by trade name, trademark, manufacturer, or otherwise, does not necessarily constitute or imply its endorsement, recommendation, or favoring by the United States Government or any agency thereof. The views and opinions of authors expressed herein do not necessarily state or reflect those of the United States Government or any agency thereof.

Reproduced from the best available copy.

Available to DOE and DOE contractors from the Office of Scientific and Technical Information

P.O. Box 62

Oak Ridge, TN 37831

Prices available from (423) 576-8401

Available to the public from the National Technical Information Service

U.S. Department of Commerce

5285 Port Royal Road

Springfield, VA 22161 


\section{Multiphase Integral Reacting Flow Computer Code (ICOMFLO): User's Guide}

S.L. Chang, S.A. Lottes, and M. Petrick

Energy Systems Division

Argonne National Laboratory, 9700 South Cass Avenue, Argonne, Illinois 60439

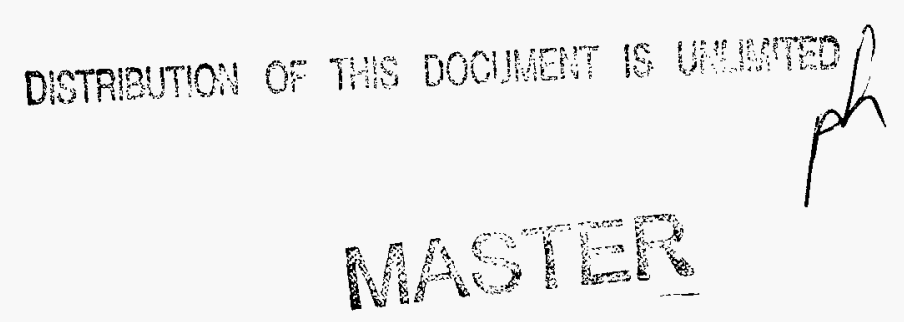

November 1997

Work sponsored by United States Department of Energy, Assistant Secretary for Fossil Energy 


\section{TABLE OF CONTENTS}

$\underline{\text { Page }}$

ACKNOWLEDGMENTS

viii

NOTATION

ix

ABSTRACT

1 INTRODUCTION

2 MATHEMATICAL FORMULATION

2.1 Gas-Phase Governing Equations ……………………....................................

2.2 Condensed-Phase Governing Equations ………................................................... 7

2.3 Boundary Conditions ………................................................................... 10

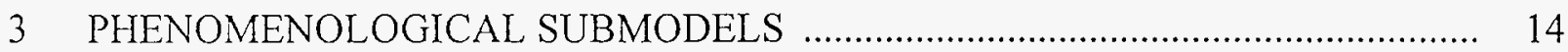

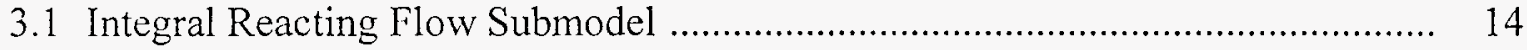

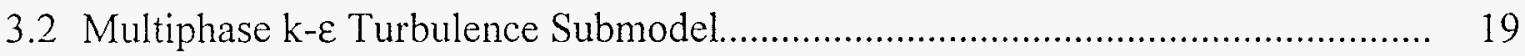

3.3 Interfacial Drag and Heat Transfer Submodel ……....................................... 22

3.4 Particle Evaporation Submodel ...................................................................... 24

4 NUMERICAL SCHEME ……….................................................................. 26

4.1 Computational Cells and Staggered Grids ...................................................... 26

4.2 Discretization of Gas-Phase Equations ……………......................................... 29

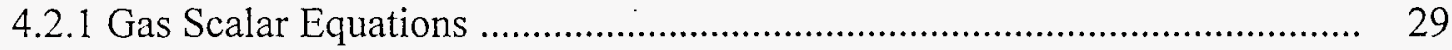

4.2.2 Gas Momentum Equations .................................................................. 33

4.2.3 Interpolation of Flow Properties .............................................................. 36

4.3 Discretization of Condensed-Phase Equations .................................................... 37

4.3.1 Particle Number Density Equation ........................................................... 37

4.3.2 Particle Momentum and Energy Equations ................................................ 39

4.4 Iterative Solution Routine ........................................................................... 40

4.4.1 Pressure Equation ............................................................................. 40

4.4.2 Pressure Correction Equation ............................................................ 42

4.4.3 Modified SIMPLER Algorithm ................................................................. 43

4.4.4 Solution of Discretized Algebraic Equations ........................................... 44

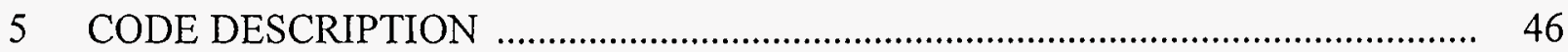

5.1 Main Control Program ................................................................................. 46

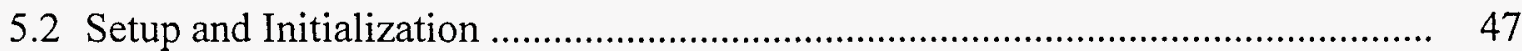

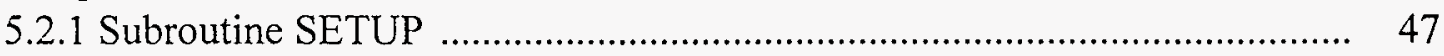

5.2.2 Subroutine GRIDS ........................................................................ 54

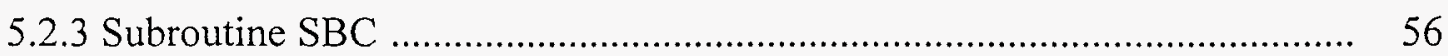

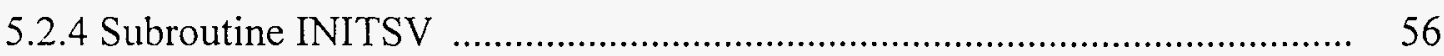

5.2.5 Subroutine HPRINT …............................................................................ 57 


\section{TABLE OF CONTENTS (cont.)}

Page

5.3 Flow Field Calculation ................................................................................ 57

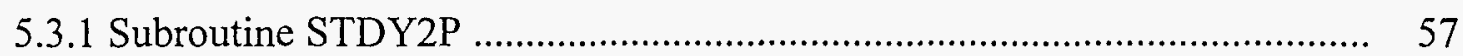

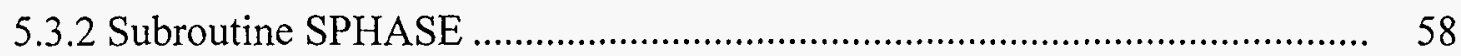

5.3.3 Subroutine GFLOW ………............................................................... 58

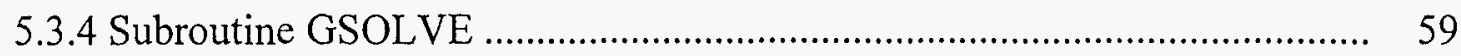

5.3.5 Subroutine DFLOW ....................................................................... 59

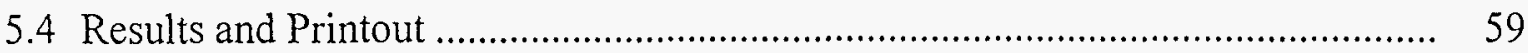

6 SAMPLE CALCULATION AND CODE VALIDATION ………........................... 60

6.1 Parameter Assignment .................................................................................. 60

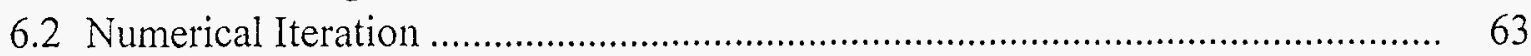

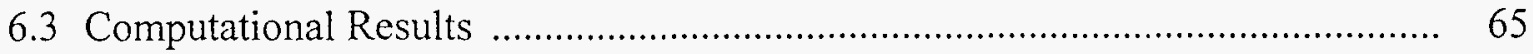

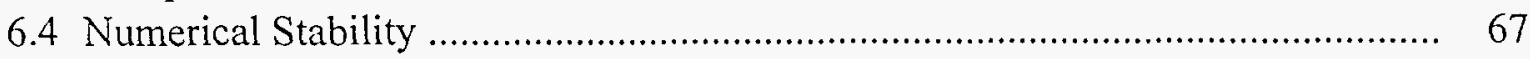

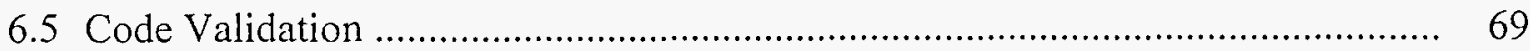

6.6 Copyright Notification ……………………….......................................... 70

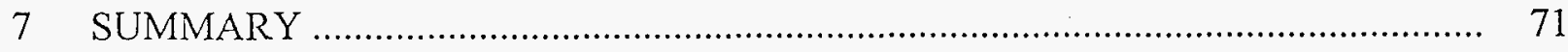

8 REFERENCES _.................................................................................... 72

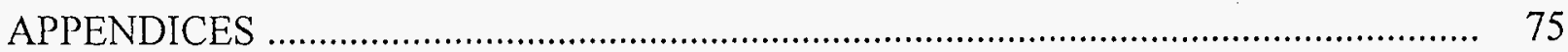




\section{LIST OF FIGURES}

No.

1 Distribution of Particle Number Density over Radius .................................................... 8

2 A Simple Combustion Channel ................................................................................ 10

3 TRW's Coal-Fired MHD Combustor System ……….................................................. 15

4 Progress of Reaction Derived from Kinetics Calculation .............................................. 18

5 Labeling of a Computational Cell ............................................................................ 26

6 Relative Position of Scalar and Momentum Cells ....................................................... 28

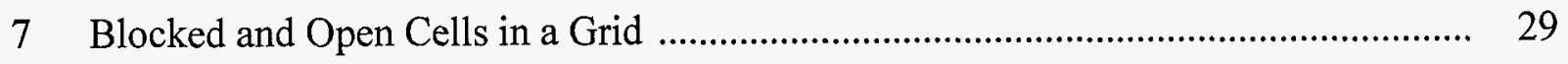

8 Labeling of an X-momentum Cell.............................................................................. 34

$9 \quad$ An Extended X-momentum Cell ............................................................................. 34

10 Labeling of a Y-momentum Cell ............................................................................. 35

11 An Extended Y-momentum Cell ............................................................................. 36

12 Labeling of a Computational Cell for Pressure Equation ............................................ 40

13 Flow Diagram of Iterative Solution Routine ............................................................... 43

14 Computational Cell Indexing of a Two-Dimensional Grid .......................................... 44

15 Flowchart of the Main Control Program ..................................................................... 47

16 Flowchart of the Setup Process .................................................................................. 48

17 A Sample Grid System with Blocked Cells ............................................................. 55

18 Flowchart of the Flow Calculation Process .............................................................. 58

19 TRW's MHD Second-Stage Combustor .................................................................... 60

20 Grid System for the Sample Combustor .................................................................... 62

21 Numerical Convergence of a Sample Calculation ......................................................... 64

22 Sample Results of Computed Flow Fields in the Combustor ....................................... 66

23 Oscillatory Numerical Instability due to Differential Approach .................................. 67

24 Converged Calculation, Using the Integral Approach ................................................. 67

25 Numerical Convergence of Integral and Differential Reaction Rate Approaches ....... 68

26 Comparison of Computed and Measured Results in a Fluid Mixing Study [6] ........... 69 


\section{LIST OF TABLES}

No.

Page

1 Properties of the Inlet Combustion Flow .................................................................... 16

2 Molecular Weights and Species Heats of the Lumped Species...................................... 16

3 Reactions and Rate Constants of the MHD Combustion Flow .................................... 17

4 Description of ICOMFLO Subroutines ………………................................................ 46

5 List of Global Constants ............................................................................................ 49

6 List of Selection Parameters ....................................................................................... 49

$7 \quad$ List of Equation Solution Indices ............................................................................ 50

8 List of Chamber Geometries ...................................................................................... 50

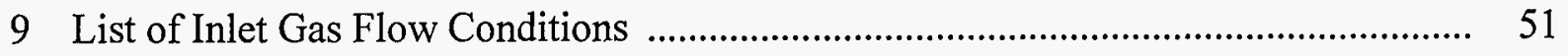

10 List of Gas Properties ............................................................................................. 52

11 List of Particle Flow Conditions ................................................................................ 52

12 List of Jet Flow Parameters ......................................................................................... 53

13 List of Turbulence Parameters ................................................................................. 53

14 List of Numerical Control Parameters ....................................................................... 54

15 List of Empirical Kinetic Constants ........................................................................... 54

16 List of Grid Parameters................................................................................................ 55

17 Computed Kinetic Constants ........................................................................................ 61

18 Sample Values of SETUP Parameters ...................................................................... 62

19 List of the ICOMFLO Files ......................................................................................... 63 


\section{LIST OF APPENDICES}

No.

Page

1 Description of ICOMFLO Variables

75

2 Kinetic Constant File for Sample Calculation

78

3 Common Block File for Sample Calculation

80

4 Printout of the Sample Calculation

81

5 Some Results of the Sample Calculation

86 


\section{ACKNOWLEDGMENTS}

This work was supported by the U.S. Department of Energy, Assistant Secretary for Fossil Energy, under Contract W-31-109-Eng-38. H. F. Chambers of Pittsburgh Energy Technology Center was program manager. N. Swift was DOE's technical project officer. D. K. Schmalzer was Argonne's program manager. W. M. Swift was Argonne's project coordinator. Encouragement from G. F. Berry during the development of the code is greatly appreciated. 


\section{NOTATION}

$\mathrm{A}\left(\mathrm{P}_{\mathrm{c}}\right) \quad$ grid cell Peclet number function, Eq. (4.11b)

a parameter of particle size distribution, Eq. (2.13), or coefficient of Eq. (4.6)

B transfer number, Eq. (3.22)

b parameter of particle size distribution, Eq. (2.13), or coefficient of Eq. (4.6)

$\mathrm{C}_{1} \quad$ turbulence empirical constant

$\mathrm{C}_{2} \quad$ turbulence empirical constant

$\mathrm{C}_{\mathrm{d}} \quad$ drag coefficient, Eq. (3.20)

$\mathrm{C}_{\mathrm{p}} \quad$ specific heat $(\mathrm{J} / \mathrm{kg}-\mathrm{K})$

$\mathrm{C}_{\mu} \quad$ turbulence empirical constant

c parameter of particle size distribution, Eq. (2.13)

D mass diffusivity $\left(\mathrm{m}^{2} / \mathrm{s}\right)$

d parameter of particle size distribution, Eq. (2.13)

$\mathrm{F}_{\mathrm{d}} \quad$ drag force $\left(\mathrm{N} / \mathrm{m}^{2}\right)$

f mass flux, Eq. (4.8a)

$\mathrm{G}_{\mu} \quad$ dissipation function $\left(1 / \mathrm{s}^{2}\right)$

$\mathrm{g}_{s} \quad$ particle number density distribution function $\left(\# / \mathrm{m}^{3}-\mathrm{m}\right)$

$\mathrm{g}_{\mathrm{x}} \quad \mathrm{x}$-component of the gravity acceleration $\left(\mathrm{m} / \mathrm{s}^{2}\right)$

$\mathrm{g}_{\mathrm{y}} \quad \mathrm{y}$-component of the gravity acceleration $\left(\mathrm{m} / \mathrm{s}^{2}\right)$

$\mathrm{H}_{\mathrm{f}} \quad$ heat of reaction $(\mathrm{J} / \mathrm{kg})$

h enthalpy $(\mathrm{J} / \mathrm{kg})$

$\mathrm{J}$ diffusive flux, Eq. (4.5a)

$\mathrm{k} \quad$ turbulent kinetic energy $(\mathrm{J} / \mathrm{kg})$

$\mathrm{L} \quad$ latent heat $(\mathrm{J} / \mathrm{kg})$

$\mathrm{L}_{c} \quad$ convective length scale in Eqs. (3.5) and (3.6), (m/s)

$\mathrm{m}_{\mathrm{d}} \quad$ mass of a particle $(\mathrm{kg} / \#)$

n particle number density $\left(\# / \mathrm{m}^{3}\right)$

$\mathrm{Nu}_{\mathrm{s}} \quad$ Nusselt number, Eq. (3.28)

$\mathrm{P} \quad$ tridiagonal matrix coefficient, Eq. (4.42b)

$\mathrm{p} \quad$ pressure $(\mathrm{Pa})$

$P_{c} \quad$ Peclet number, Eq. (4.11c)

Q tridiagonal matrix coefficient, Eq. (4.42d)

$\mathrm{q} \quad$ interfacial heat flux $(\mathrm{J} / \mathrm{s})$

$\mathrm{R}$ universal gas constant $(\mathrm{J} / \mathrm{kmol}-\mathrm{K})$

$\mathrm{Re}_{\mathrm{s}} \quad$ Reynolds number, Eq. (3.21)

$r_{m} \quad$ mean particle radius $(\mu \mathrm{m})$

$r \quad$ particle radius $(\mu \mathrm{m})$

$\mathrm{S} \quad$ Source term in governing equations

Sc Schmidt number, Eq. (3.29)

$\mathrm{T}$ temperature $(\mathrm{K})$

$\mathrm{T}_{\mathrm{a}} \quad$ adiabatic flame temperature $(\mathrm{K})$

$\mathrm{T}_{\mathrm{b}} \quad$ boiling temperature $(\mathrm{K})$

$\mathrm{t} \quad$ time $(\mu \mathrm{s})$

$\mathrm{u} \quad$ velocity component in the $\mathrm{x}$-direction $(\mathrm{m} / \mathrm{s})$

$\mathrm{u}^{\prime} \quad$ velocity fluctuation $(\mathrm{m} / \mathrm{s})$

$\mathrm{v} \quad$ velocity component in the $y$-direction $(\mathrm{m} / \mathrm{s})$

$\mathrm{V}_{\mathrm{c}}$ convective velocity scale in Eqs. (3.5) and (3.6), (m/s)

w molecular weight $(\mathrm{kg} / \mathrm{kmol})$

$\mathrm{x} \quad \mathrm{x}$ coordinate $(\mathrm{m})$

$y \quad y$ coordinate $(m)$

$Y_{f} \quad$ concentration of fuel species $(\mathrm{kg} / \mathrm{kg})$

$\mathrm{Y}_{\mathrm{f}, \mathrm{o}} \quad$ initial fuel concentration $(\mathrm{kg} / \mathrm{kg})$ 
NOTATION (Cont.)

$Y_{\mathrm{c}} \quad$ concentration of inert species $(\mathrm{kg} / \mathrm{kg})$

$\mathrm{Y}_{0} \quad$ concentration of oxidizer species $(\mathrm{kg} / \mathrm{kg})$

$Y_{p} \quad$ concentration of product species $(\mathrm{kg} / \mathrm{kg})$

$\mathrm{Y}_{\mathrm{r}} \quad$ reference concentration $(\mathrm{kg} / \mathrm{kg})$

\section{Greek Symbols}

$\Gamma_{n} \quad$ number density diffusivity (Pa-s)

$\Gamma_{\mathrm{p}}$ diffusivity for enthalpy, species, and richness (Pa-s)

$\varepsilon \quad$ turbulent dissipation rate $(\mathrm{J} / \mathrm{kg}-\mathrm{s})$

$\lambda$ gas conductivity $(\mathrm{J} / \mathrm{s}-\mathrm{m}-\mathrm{K})$

$\lambda_{i} \quad$ macro turbulent length scale $(\mathrm{m})$

$\mu \quad$ gas viscosity (Pa-s)

$\mu_{\mathrm{e}} \quad$ effective viscosity (Pa-s)

$\theta \quad$ void fraction

$\rho$ density $\left(\mathrm{kg} / \mathrm{m}^{3}\right)$

$\Phi$ richness

$\phi \quad$ stoichiometric ratio

$\sigma_{k} \quad$ turbulence empirical constant

$\sigma_{p} \quad$ property diffusivity constant

$\sigma_{\varepsilon} \quad$ turbulence empirical constant

$\xi \quad$ general variable

$\zeta \quad$ extent of reaction

$\varphi \quad$ particle dispersion function, Eq. (3.16)

\section{Subscripts}

E east node

e inert species or east interface

f fuel species

i particle lump i

$\mathrm{N}$ north node

n north direction

o oxidizer species

$\mathrm{p} \quad$ product species or node point of interest

$\mathrm{r}$ reference

$S$ south node

$\mathrm{s}$ condensed phase or south interface

$\mathrm{t}$ turbulent property

W west node

w west interface

$\delta \quad$ slip property 


\title{
Multiphase Integral Reacting Flow Computer Code (ICOMFLO): User's Guide
}

by

\author{
S. L. Chang, S. A. Lottes, and M. Petrick
}

\begin{abstract}
A copyrighted computational fluid dynamics computer code, ICOMFLO, has been developed for the simulation of multiphase reacting flows. The code solves conservation equations for gaseous species and droplets (or solid particles) of various sizes. General conservation laws, expressed by elliptic-type partial differential equations, are used in conjunction with rate equations governing the mass, momentum, enthalpy, species, turbulent kinetic energy, and turbulent dissipation. Associated phenomenological submodels of the code include integral combustion, two-parameter turbulence, particle evaporation, and interfacial submodels. A newly developed integral combustion submodel replacing an Arrhenius-type differential reaction submodel has been implemented to improve numerical convergence and enhance numerical stability. A two-parameter turbulence submodel is modified for both gas and solid phases. An evaporation submodel treats not only droplet evaporation but size dispersion. Interfacial submodels use correlations to model interfacial momentum and energy transfer.

The ICOMFLO code solves the governing equations in three steps. First, a staggered grid system is constructed in the flow domain. The staggered grid system defines gas velocity components on the surfaces of a control volume, while the other flow properties are defined at the volume center. A blocked cell technique is used to handle complex geometry. Then, the partial differential equations are integrated over each control volume and transformed into discrete difference equations. Finally, the difference equations are solved iteratively by using a modified SIMPLER algorithm. The results of the solution include gas flow properties (pressure, temperature, density, species concentration, velocity, and turbulence parameters) and particle flow properties (number density, temperature, velocity, and void fraction).

The ICOMFLO code is written in the FORTRAN language and can be operated on a CRAY supercomputer, a VAX minicomputer, or a personal computer. The code has been used in many engineering applications, such as coal-fired combustors, air-breathing propulsion engines, and internal combustion engines. The code has been partially validated by favorable comparisons of calculation results with available experimental data. A sample calculation is given to illustrate the use of the ICOMFLO code.
\end{abstract}

\section{INTRODUCTION}

Computer simulation of a reacting flow provides a powerful tool to use in the development of an advanced combustor or in the improvement of an existing design. Computer simulation can output the values of all flow properties at points on a grid mesh covering the entire flow field. The results can be readily used for troubleshooting, such as identifying points of rapid particle deposition on a wall, or to search for optimum operating conditions, including maximizing efficiency or power density. Use of computer simulation can speed up the 
development cycle by providing information to use in optimizing a design, and by reducing the number of prototypes that need to be built and tested. Computer simulation can be used to investigate all kinds of alternatives in combustor design, from different fuel/air mixtures to different combustor geometries, and to explore new design ideas quickly.

The multiphase integral combustion flow (ICOMFLO) computer code was developed at Argonne National Laboratory (ANL) in a study to investigate flow characteristics in a magnetohydrodynamic (MHD) power generation system. The ICOMFLO code is a general computational fluid dynamics (CFD) computer code. The computer code was originally developed to study the effects and implications of group combustion theory developed by Chiu and Liu [1]. In 1983, Zhou of the University of Illinois at Chicago developed a two-dimensional steady-state spray combustion computer code based on the macro-scale group combustion theory [2]. It was called GEMCHIP (General Elliptic Multiphase Combustion, Heat-transfer, and Interdiffusion Program). In 1987, Jiang applied the GEMCHIP code for a simulation of airbreathing propulsion combustors [3]. Also in 1987, Chang and Wang coupled a thermal radiation model with the GEMCHIP code to investigate the interactions between soot and gas radiation and spray combustion in a direct-injection-type diesel engine [4]. In 1989, Lottes extended the GEMCHIP code to transient calculations to assess group combustion mode transitions brought on by changing inlet droplet number density in a simple cylindrical combustor [5].

From 1989 to 1994, Chang and Lottes of ANL revised the GEMCHIP code substantially for a series of computer simulations for the coal-fired MHD second-stage combustor [6-17]. Many of these revisions incorporated critical new submodels and features necessary for simulation of the MHD combustor application. Among the most important of these enhancements were fixing the equation solver to preserve symmetry in the computation of symmetric steady-state flows, modifying the code to handle grids of arbitrary geometry by using a blocked cell technique, adding a model for deposition of particles or droplets on a wall boundary, providing for jet injection openings in the walls, and adding dynamic grid modification (during iterative computation) of cells spanning jet openings to allow specification of both jet velocity and mass flow rate as jet boundary conditions while still preserving effects of gas compressibility in the jet entry region. The most significant modification of the code was developed in 1993, when Chang and Lottes added to the combustion code a new integral combustion submodel replacing an Arrhenius-type differential combustion submodel. The new integral combustion submodel greatly improved numerical convergence and stability of the code and made possible many computations that would have been numerically unstable and either diverged or oscillated without converging under the old combustion model [16]. Since then, the computer code has been called ICOMFLO. In 1994, ICOMFLO was extended to three dimensions in space to investigate the deposition patterns of seed particles in a deswirl section of the MHD power generation system [18].

The two-dimensional steady-state version of the ICOMFLO computer code is described in this report. The mathematical formulation of the conservation equations is described in Chapter 2. The associated phenomenological submodels are presented in Chapter 3. Numerical methodologies are given in Chapter 4. The description of the ICOMFLO code is contained in Chapter 5. A sample program is given in Chapter 6 to illustrate the use of the code. 


\section{MATHEMATICAL FORMULATION}

Flow properties, such as velocity, temperature, density, pressure, and species concentration, are design parameters for advanced flow systems; therefore, they are important in the analysis of a multiphase combustion flow. The flow properties are governed by the laws of physics, including conservation of mass (both globally and over multiple chemical species), momentum, and energy. Partial differential equations are derived for the flow to satisfy the conservation laws. The flow properties are to be solved by means of these conservation equations. For the gas phase, separate nondifferential equations for some property states (such as density, specific heat, etc.), one of the chemical species (because the mass fractions must total to one, one of the chemical species is constrained by the sum of the others), and turbulent diffusivity are calculated from the results of conservation equation solutions. The condensed phase can be either liquid or solid. For convenience in discussion, the solid phase is used to represent the condensed phase in this report. For the condensed phase, the particles are divided into a number of size groups and the conservation equations are derived for each size group. The equations used to solve for flow properties are called governing equations. Some governing equations can be derived theoretically from the laws of physics, while others rely in part on empirical correlations or phenomenological submodels. This chapter presents the theoretical governing equations, and the next chapter discusses the phenomenological submodels.

\subsection{Gas-Phase Governing Equations}

For the analysis of a combustion flow, gas flow properties to be solved include velocity components in the $x$ - and $y$-directions $(u, v)$, pressure $(p)$, enthalpy $(h)$, temperature $(T)$, density $(\rho)$, and concentration of chemical species $(Y)$. The governing equations for these flow properties are conservation equations of mass, momentum, energy, and species concentration, plus the equations of state. The number of chemical species can be one or many, depending on the nature of the flow. For example, four species (fuel, oxidizer, product, and inert) are used in the analysis of the combustion process in a coal-fired MHD combustor [10] and five species (fuel, oxidizer, product, inert, and potassium) are used in the analysis of the vaporization of seed material in the MHD combustor [12]. In principle, one can include as many species as needed in an analysis by adding the species equations to the solution process. However, the addition of the species equations not only requires a larger memory space in a computer and longer computational time, but it often also causes numerical problems that prevent one from obtaining a solution. In the following discussion, a four-species flow including fuel, oxidizer, product, and inert is assumed. Ten governing equations are needed to solve for 10 flow properties ( $u, v, p, h$, $T, \rho, Y_{f}, Y_{o}, Y_{p}$, and $Y_{e}$ ). Note that $Y_{f}, Y_{o}, Y_{p}$, and $Y_{e}$ are concentrations of fuel, oxidizer, product, and inert species, respectively. The governing equations are presented in the following: the continuity Eq. 2.1, the x-momentum Eq. 2.2, the y-momentum Eq. 2.3, the energy Eq. 2.4, the species state Eq. 2.5, the fuel species conservation Eq. 2.6, the oxidizer species conservation Eq. 2.7, the inert species conservation Eq. 2.8, the perfect gas state Eq. 2.9, and the caloric state Eq. 2.10. 
Gas continuity equation:

$$
\frac{\partial}{\partial x}(\theta \rho u)+\frac{\partial}{\partial y}(\theta \rho v)=S_{1}
$$

Gas X-momentum equation:

$$
\frac{\partial}{\partial x}\left(\theta \rho u u-\mu_{e} \frac{\partial u}{\partial x}\right)+\frac{\partial}{\partial y}\left(\theta \rho v u-\mu_{e} \frac{\partial u}{\partial y}\right)=\rho g_{x}-\theta \frac{\partial p}{\partial x}+\frac{\partial}{\partial x}\left(\mu_{e} \frac{\partial u}{\partial x}\right)+\frac{\partial}{\partial y}\left(\mu_{e} \frac{\partial v}{\partial x}\right)+S_{u, e}+S_{u, d}
$$

where the effective viscosity $\mu_{\mathrm{e}}$ is defined as

$$
\mu_{\mathrm{e}}=\mu_{\mathrm{t}}+\mu
$$

Gas y-momentum equation:

$$
\frac{\partial}{\partial x}\left(\theta \rho u v-\mu_{e} \frac{\partial v}{\partial x}\right)+\frac{\partial}{\partial y}\left(\theta \rho v v-\mu_{e} \frac{\partial v}{\partial y}\right)=\rho g_{y}-\theta \frac{\partial p}{\partial y}+\frac{\partial}{\partial x}\left(\mu_{e} \frac{\partial u}{\partial y}\right)+\frac{\partial}{\partial y}\left(\mu_{e} \frac{\partial v}{\partial y}\right)+S_{v, e}+S_{v, d}
$$

Gas energy conservation equation:

$$
\frac{\partial}{\partial x}\left(\theta \rho u h-\Gamma_{p} \frac{\partial h}{\partial x}\right)+\frac{\partial}{\partial y}\left(\theta \rho v h-\Gamma_{p} \frac{\partial h}{\partial y}\right)=\theta \mu_{e} G_{\mu}+S_{h, c}+S_{h, d}
$$

where the dissipation function $\mathrm{G}_{\mu}$ is

$$
G_{\mu}=2\left[\left(\frac{\partial u}{\partial x}\right)^{2}+\left(\frac{\partial v}{\partial y}\right)^{2}\right]+\left[\frac{\partial u}{\partial y}+\frac{\partial v}{\partial x}\right]^{2}
$$

and the property diffusivity $\Gamma_{\mathrm{p}}$ is defined as

$$
\Gamma_{p}=\theta\left(\mu_{t}+\mu\right) / \sigma_{p}
$$

Total gas species conservation equation:

$$
Y_{f}+Y_{o}+Y_{p}+Y_{i}=1
$$

Fuel species conservation equation:

$$
\frac{\partial}{\partial x}\left(\theta \rho u Y_{f}-\Gamma_{p} \frac{\partial Y_{f}}{\partial x}\right)+\frac{\partial}{\partial y}\left(\theta \rho v Y_{f}-\Gamma_{p} \frac{\partial Y_{f}}{\partial y}\right)=S_{Y, f}
$$

Oxidizer species conservation equation:

$$
\frac{\partial}{\partial x}\left(\theta \rho u Y_{o}-\Gamma_{p} \frac{\partial Y_{o}}{\partial x}\right)+\frac{\partial}{\partial y}\left(\theta \rho v Y_{o}-\Gamma_{p} \frac{\partial Y_{o}}{\partial y}\right)=S_{Y, o}
$$


Inert species conservation equation:

$$
\frac{\partial}{\partial x}\left(\theta \rho u Y_{e}-\Gamma_{p} \frac{\partial Y_{e}}{\partial x}\right)+\frac{\partial}{\partial y}\left(\theta \rho v Y_{e}-\Gamma_{p} \frac{\partial Y_{e}}{\partial y}\right)=0
$$

State equation of perfect gas:

$$
p=\rho R T / w
$$

where

$$
w=\frac{1}{Y_{f} / w_{f}+Y_{o} / w_{o}+Y_{p} / w_{p}+Y_{e} / w_{e}}
$$

Gas-phase caloric equation of state:

$$
h=C_{p}\left(T-T_{r}\right)
$$

where

$$
C_{p}=Y_{f} C_{p, f}+Y_{o} C_{p, o}+Y_{p} C p_{p, p}+Y_{e} C_{p, e}
$$

The continuity equation, Eq. 2.1, is a first-order partial differential equation. There are two terms on the left-hand side of the equation representing the net change of mass fluxes in the $\mathrm{x}$ and $y$-directions. The right-hand side of the equation is a source term $S_{1}$ representing the addition of gas mass from particle melting and subsequent evaporation. The formulation of the particle evaporation rate will be given in Chapter 3. Generally speaking, the continuity equation is not solved directly because of the sensitivity of the pressure to small changes in the momentum field. Since the gas in this application is relatively hard to compress, a small mass imbalance in a numerical calculation can result in a steep change of pressure and cause the calculation to fail. Therefore, numerical stability is enhanced when the pressure field is solved for first. By substituting the momentum equations, suitably rearranged, into the continuity equation, the continuity equation can be transformed into a pressure equation for solving the pressure field. The pressure equation will be derived in Chapter 4 .

The momentum, energy, and species conservation equations, Eqs. 2.2, 2.3, 2.4, 2.6, 2.7, and 2.8, are second-order partial differential equations expressed in a common format with two convective terms (first and third) and-two diffusive terms (second and fourth) on the left-hand side and the source terms on the right-hand side. A convective term of a property governing equation $\left(\mathrm{u}, \mathrm{v}, \mathrm{h}, \mathrm{Y}_{\mathrm{f}}, \mathrm{Y}_{\mathrm{o}}\right.$, or $\left.\mathrm{Y}_{\mathrm{c}}\right)$ is the net change of the property convective flux, the product of the gas mass flux ( $\theta \rho u$ or $\theta \rho v$ ) and the property. A diffusive term is the net change of a property diffusive flux, the product of a diffusivity coefficient $\left(\mu_{\mathrm{e}}\right.$ or $\left.\Gamma_{\mathrm{p}}\right)$ and the gradient of the property. The diffusion of a property includes both laminar and turbulent effects. The diffusivity coefficient for the momentum equations is an effective viscosity $\mu_{\mathfrak{e}}$, defined as the sum of the turbulent viscosity $\mu_{\mathrm{t}}$ and gas viscosity $\mu$ as shown in Eq. 2.2a. The turbulent viscosity is a flow property and needs to be determined from a separate turbulence submodel; the turbulence 
submodel will be presented in Chapter 3. The diffusivity coefficient for the energy and species equations is a property diffusivity $\Gamma_{\mathrm{p}}$, defined as the product of the effective viscosity, the void fraction, and an empirical constant $\sigma_{\mathrm{p}}$ as shown in Eq. 2.4a. An empirical constant of 0.7 used by Zhou and Chiu [2] is used here. Since the diffusivity coefficients are positive, the momentum, energy, and species equations are all elliptic-type second-order partial differential equations. These governing equations need a complete description of the boundary conditions to obtain a solution.

Source terms of the momentum, energy, and species governing equations are grouped on the right-hand side of the equation. A source term becomes a sink term when the value is negative. Each of the momentum equations, Eqs. 2.2 and 2.3, has six source terms. The first term $\left(g_{x}\right.$ or $\left.g_{y}\right)$, representing the portion of the gravitational constant acting in the $x-$ and $y$ directions, respectively, is the gravity effect. The second term $(-\partial \mathrm{p} / \partial \mathrm{x}$ or $-\partial \mathrm{p} / \partial \mathrm{y})$ is the pressure gradient effect. The third and the fourth terms are the remaining viscous terms of significance, besides the two diffusive terms in the left-hand of the equation. The reason for separating these viscous terms is to maintain a common format on the left-hand side of the equation for all partial differential equations and to simplify the formulation and programming of the equation solver. The fifth term $\left(\mathrm{S}_{\mathrm{u}, \mathrm{e}}\right.$ or $\left.\mathrm{S}_{\mathrm{v}, \mathrm{e}}\right)$ is the momentum change due to the added mass of the particle evaporation. The sixth term $\left(\mathrm{S}_{\mathrm{u}, \mathrm{d}}\right.$ or $\left.\mathrm{S}_{\mathrm{v}, \mathrm{d}}\right)$ is the momentum change due to the interfacial drag forces. The energy equation has three source terms, including the effects of viscous dissipation $\mathrm{G}_{\mu}$, heat of combustion $\mathrm{S}_{\mathrm{h}, \mathrm{c}}$, and heat exchange between phases $\mathrm{S}_{\mathrm{h}, \mathrm{d}}$. Source terms in the fuel and oxidizer species conservation equations $\left(\mathrm{S}_{\mathrm{Y}, \mathrm{f}}\right.$ and $\left.\mathrm{S}_{\mathrm{Y}, 0}\right)$ arise out of the consumption or production of species in chemical reactions. No source term is needed for the inert species conservation equation. The formulation of the source terms $\left(S_{u, e}, S_{v, e}, S_{u, d}, S_{v, d}, S_{h, c}, S_{h, d}\right.$, and $\left.S_{Y, f}\right)$ is given in Chapter 3.

It is well known that a source term in the governing partial differential equations (especially source terms that are positive and functions of the dependent variable) can cause severe numerical instability because of the overshooting problem, especially when the time scale of the source term is much smaller than the flow time scale. In a combustion flow, the reaction time scale for the source term is typically several orders of magnitude smaller than the flow time scale. Therefore, it is desirable to eliminate a source term when possible. Since the consumption rates of the fuel and the oxidizer in a combustion process are related by a chemical reaction, one of the source terms $\left(S_{Y, f}\right.$ and $\left.S_{Y, o}\right)$ can be eliminated by defining a new derived property and a new governing equation for the derived property. A new derived property called richness $\Phi$ is defined as:

$$
\Phi=\frac{Y_{f}-Y_{o} / \phi}{1+Y_{r} / \phi}+\frac{Y_{r}}{\phi+Y_{r}}
$$


where $\phi$ is the stoichiometric ratio of the chemical reaction and $Y_{r}$ is a reference species concentration. Note that the stoichiometric ratio is defined as the mass ratio of oxidizer to fuel at the stoichiometric condition. Usually, the inlet oxidizer concentration is used as the reference species concentration.

By dividing Eq. 2.7 by the stoichiometric ratio and subtracting the result from Eq. 2.6, a new transport equation, Eq. 2.12, is derived for the newly defined property, richness. The new equation has no source term. Therefore, computations are more stable if it is used in place of the oxidizer species conservation equation, Eq. 2.7, in solving the governing equations.

Transport equation of richness:

$$
\frac{\partial}{\partial x}\left(\theta \rho u \Phi-\Gamma_{\mathrm{p}} \frac{\partial \Phi}{\partial \mathrm{x}}\right)+\frac{\partial}{\partial \mathrm{y}}\left(\theta \rho v \Phi-\Gamma_{\mathrm{p}} \frac{\partial \Phi}{\partial \mathrm{y}}\right)=0
$$

With a four-species flow assumed, Eq. 2.5 is the species state equation. If it is assumed that the gas is a perfect ideal gas, Eqs. 2.9 and 2.10 are the state equations. The compositions of the species and their molecular weights $\left(\mathrm{w}_{\mathrm{f}}, \mathrm{w}_{\mathrm{o}}, \mathrm{w}_{\mathrm{p}}\right.$, and $\left.\mathrm{w}_{\mathrm{e}}\right)$ and specific heats $\left(\mathrm{Cp}_{\mathrm{f}}, \mathrm{Cp}_{\mathrm{o}}, \mathrm{Cp}_{\mathrm{p}}\right.$, and $\mathrm{Cp}_{\mathrm{e}}$ ) are given in the integral reaction submodel section in Chapter 3.

\subsection{Condensed-Phase Governing Equations}

Experiments show that particles have nonuniform size in a spray. For a typical spray, a Gamma function is usually used to describe the particle size distribution. A particle number density distribution function $\mathrm{g}_{\mathrm{s}}(\mathrm{r})$ is defined as Eq. 2.13:

$$
g_{s}(r)=b r^{c} \exp \left(-a r^{d}\right)
$$

The particle number density distribution function employs four parameters, a, b, c, and d. Two of the parameters are related to the total number density and mean radius and the other two need to be determined empirically. The total particle number density $\mathrm{n}_{0}$ and the volume mean radius $r_{m}$ can be derived from the particle distribution function. Equation 2.14 shows the formula of the total number density, and Eq. 2.15 shows the formula of the volume mean radius.

$$
\begin{aligned}
& n_{o}=\int_{0}^{\infty} g_{s}(r) d r \\
& r_{m}=\left[\frac{1}{n_{0}} \int_{0}^{\infty} r^{3} g_{s}(r) d r\right]^{1 / 3}
\end{aligned}
$$

On the assumption that $c$ and $d$ are empirical parameters and are both equal to 4 , parameters a and $b$ can be expressed in terms of $n_{o}$ and $r_{m}$, and the particle number density distribution function becomes 


$$
\frac{\mathrm{g}_{\mathrm{s}} \mathrm{r}_{\mathrm{m}}}{\mathrm{n}_{\mathrm{o}}}=5.2\left(\mathrm{r} / \mathrm{r}_{\mathrm{m}}\right)^{4} \exp \left[-1.14\left(\mathrm{r} / \mathrm{r}_{\mathrm{m}}\right)^{4}\right]
$$

Equation 2.16 shows the particle number density distribution function expressed in a dimensionless form. The particle number density distribution function is represented by a solid line in Fig. 1. It is a typical bell-shaped normal distribution. As the particle radius increases, the number density function increases from zero at zero radius to a peak $\left(g=1.66 \mathrm{n}_{0} / \mathrm{r}_{\mathrm{m}}\right)$ at a radius $\mathrm{r}=0.97 \mathrm{r}_{\mathrm{m}}$ and decreases afterward. The width of the distribution can be adjusted by changing the values of $\mathrm{c}$ and $\mathrm{d}$.

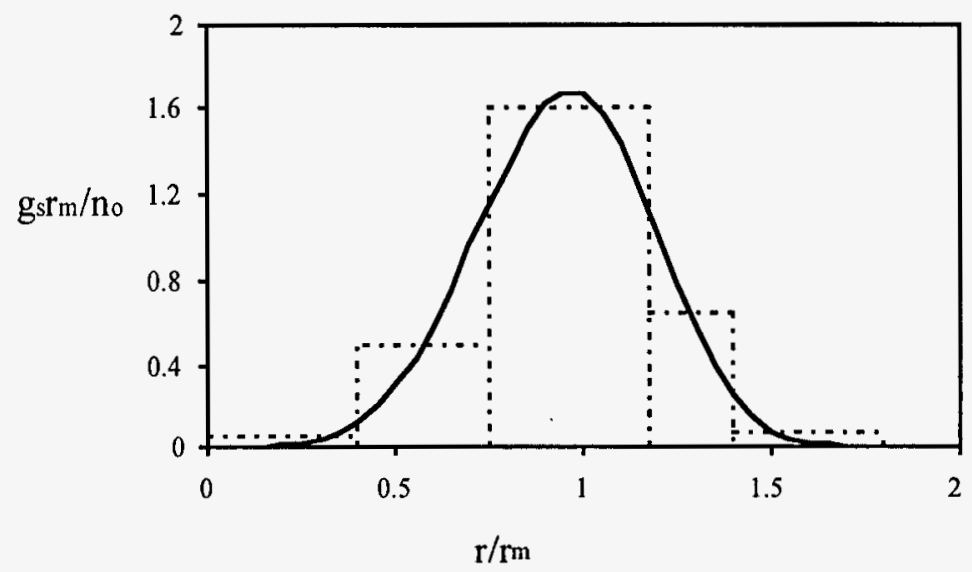

Figure 1 Distribution of Particle Number Density over Radius

In a numerical calculation, the particle size distribution function is simplified by lumping particles into several size groups. If particles are divided into $\mathrm{k}$ lumps, the number density of the $i^{\text {th }}$ lump can be defined as

$$
\mathrm{n}_{\mathrm{i}}=\int_{\mathrm{r}_{\mathrm{i}-1 / 2}}^{\mathrm{r}_{\mathrm{i}+1 / 2}} \mathrm{~g}_{\mathrm{s}}\left(\mathrm{r}_{\mathrm{s}}\right) \mathrm{dr} \mathrm{r}_{\mathrm{s}}, \quad \mathrm{i}=1, \cdots \mathrm{k}
$$

where $r_{i-1 / 2}$ and $r_{i+1 / 2}$ are the lower and upper bounds of radius for the $i^{\text {th }}$ size lump of particles, respectively. The mean radius of the $i^{\text {th }}$ lump of particles is defined as $r_{i}=\left(r_{i-1 / 2}+r_{i+1 / 2}\right) / 2$. Note that $r_{1 / 2}$ is zero and $r_{k+1 / 2}$ is infinity. In practice, $g_{s}\left(r_{s}\right)$ is always defined so that the improper integral for the $\mathrm{k}^{\text {th }}$ size group converges, and a maximum particle size always exists, usually determined by equipment geometry, such as nozzle size, etc. A 5-lump particle number density distribution is shown in Fig. 1, represented by the dashed line rectangles. Note that after discretization of the distribution, the maximum particle radius is less than twice the volume mean particle radius.

For the analysis of a multiphase flow, important particle flow properties include particle number density $(n)$, velocity components in the $x$ - and $y$-directions $\left(u_{s}, v_{s}\right)$, and temperature $\left(T_{s}\right)$. The governing equations are derived from the conservation laws of mass, momentum, and energy. 
Particle mass conservation equation (lump i):

$$
\frac{\partial}{\partial x}\left[m_{d, i} n_{i} u_{i}-\Gamma_{n, i} \frac{\partial\left(m_{d, i} n_{i}\right)}{\partial x}\right]+\frac{\partial}{\partial y}\left[m_{d, i} n_{i} v_{i}-\Gamma_{n, i} \frac{\partial\left(m_{d, i} n_{i}\right)}{\partial y}\right]=S_{n, i}
$$

Mass of a particle (lump i):

$$
\mathrm{m}_{\mathrm{di}}=\rho_{\mathrm{s}} \frac{4 \pi \mathrm{r}_{\mathrm{i}}^{3}}{3}
$$

By dividing Eq. 2.18 by the particle mass $\mathrm{m}_{\mathrm{d}, \mathrm{i}}$, the particle mass conservation equation is converted to a particle number density equation (lump i):

$$
\frac{\partial}{\partial x}\left(n_{i} u_{i}-\Gamma_{n, i} \frac{\partial n_{i}}{\partial x}\right)+\frac{\partial}{\partial y}\left(n_{i} v_{i}-\Gamma_{n, i} \frac{\partial n_{i}}{\partial y}\right)=S_{n, i} / m_{d i}
$$

Particle $x$-momentum equation (lump i):

$$
\left[\frac{\partial}{\partial x}\left(n_{i} u_{i} u_{i}\right)+\frac{\partial}{\partial y}\left(n_{i} v_{i} u_{i}\right)\right]-u_{i}\left[\frac{\partial}{\partial x}\left(n_{i} u_{i}\right)+\frac{\partial}{\partial y}\left(n_{i} v_{i}\right)\right]=S_{u, i} / m_{d, i}
$$

Particle y-momentum equation (lump i):

$$
\left[\frac{\partial}{\partial x}\left(n_{i} u_{i} v_{i}\right)+\frac{\partial}{\partial y}\left(n_{i} v_{i} v_{i}\right)\right]-v_{i}\left[\frac{\partial}{\partial x}\left(n_{i} u_{i}\right)+\frac{\partial}{\partial y}\left(n_{i} v_{i}\right)\right]=S_{v, i} / m_{d, i}
$$

Particle energy conservation equation (lump i):

$$
\left[\frac{\partial}{\partial x}\left(n_{i} u_{i} T_{i}\right)+\frac{\partial}{\partial y}\left(n_{i} v_{i} T_{i}\right)\right]-T_{i}\left[\frac{\partial}{\partial x}\left(n_{i} u_{i}\right)+\frac{\partial}{\partial y}\left(n_{i} v_{i}\right)\right]=S_{T, i} / m_{d, i}
$$

The particle governing equations are all partial differential equations. Note that they have already been discretized over the particle size space, and that the particle size space is really another dimension in the mathematical space over which the problem is defined. Recognizing this aspect of the multiphase flow modeling problem is important in terms of conserving computational resources (both memory and computational time). Sensitivity studies are required, similar to grid sensitivity studies, to find the minimum number of particle size groups that will give solution independence to acceptable accuracy. Equation 2.18 is the particle mass conservation equation, balancing the particle mass in a control volume or in the neighborhood of a point when the volume approaches zero in the limit. Particle mass fluxes out of a differential control volume should be equal to the rate of local particle mass change. The equation is arranged to have four flux terms on the left-hand side of the equal sign and one source term on the right-hand side. The first and the third flux terms are the convective terms representing the net changes of the particle mass flow $\left(\mathrm{m}_{\mathrm{d}, \mathrm{i}} \mathrm{n}_{\mathrm{i}} \mathrm{u}_{\mathrm{i}}\right.$ and $\left.\mathrm{m}_{\mathrm{d}, \mathrm{i}} \mathrm{n}_{\mathrm{i}} \mathrm{v}_{\mathrm{i}}\right)$. Note that $\mathrm{m}_{\mathrm{d}, \mathrm{i}}$ is the mean mass of an $\mathrm{i}^{\text {th }}$ lump particle defined in Eq. 2.18a. The second and the fourth flux terms are the diffusive terms representing the net change of diffusive fluxes, the product of a turbulent diffusivity coefficient $\left(\Gamma_{\mathrm{n}, \mathrm{i}}\right)$ and the gradient of the particle mass $\left(\mathrm{m}_{\mathrm{d}, \mathrm{i}} \mathrm{n}_{\mathrm{i}}\right)$. The diffusivity coefficient needs to be determined in a separate turbulence submodel. The source term $m_{d, i} S_{n, i}$ accounts for the change of particle mass due to the evaporation (combined with an initial melting step in the model) and results in a shift in the particle size distribution toward smaller sizes. Since the particle mass $\mathrm{m}_{\mathrm{d}, \mathrm{i}}$ is a constant in Eq. 2.18 , the equation can be divided by $\mathrm{m}_{\mathrm{d}, \mathrm{i}}$ to yield a particle number density, Eq. 2.19. Equations 2.20 and 2.21 are momentum equations balancing the net change of particle momentum fluxes with evaporation momentum loss and interfacial drag force. 
Equation 2.20 is the $\mathrm{x}$-momentum equation and Eq. 2.21 is the y-momentum equation. Equation 2.22 is the particle energy conservation equation balancing the net change of particle energy fluxes with evaporation enthalpy loss and interfacial heat transfer. These equations, derived after being divided by the particle mass, are arranged to have two convective flux terms (the first and the second) and two evaporation loss terms (the third and the fourth) on the lefthand side of the equality sign and one source term on the right-hand side.

\subsection{Boundary Conditions}

Since the governing equations of gas momentum, energy, and species are elliptic-type second-order partial differential equations, a complete description of the boundary conditions is needed to solve the equations. For illustration, a schematic of a simulated combustor is shown in Fig. 2. The combustor has three types of boundaries: inlet, exit, and wall. In the following discussion, it is assumed that the inlet plane is at $\mathrm{x}=0$; the exit plane is at $\mathrm{x}=1$; and the walls are at $\mathrm{y}= \pm 1 / 2$.

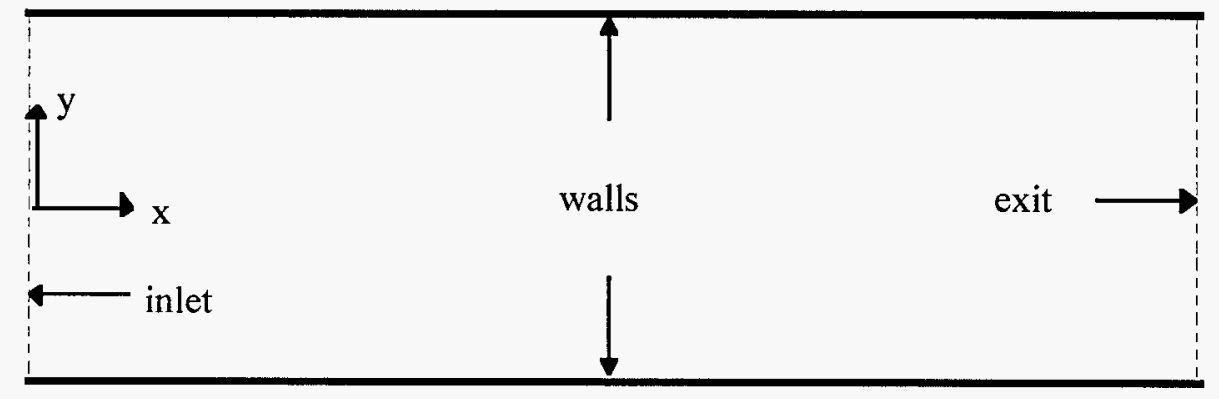

Figure 2 A Simple Combustion Channel

Gas flow properties to be specified at the inlet include the velocity components ( $u$ and $v$ ), species concentrations $\left(Y_{f}, Y_{0}, Y_{p}\right.$, and $\left.Y_{e}\right)$ and two thermodynamic properties ( $T$ and $p$ ). Note that the thermodynamic properties can be any pair of independent properties. A reference pressure $p_{o}$ is set at the center point of the inlet plane. Since velocity, pressure, and density are related by Bernoulli's equation along streamlines, with the velocity and temperature set as boundary conditions at the inlet, pressure is not a free inlet variable, and slight adjustments to the inlet reference pressure and pressure distribution are made to satisfy this constraint during the solution procedure. Particle flow inlet properties include number density $\left(\mathrm{n}_{\mathrm{i}}\right)$, velocity components $\left(u_{i}\right.$ and $\left.v_{i}\right)$, and temperature $\left(T_{i}\right)$ for all size lumps of particles. The inlet number density for each lump of particles can be specified from experimental data or the empirical formula, Eq. 2.16. An inlet property is specified as a spatial distribution over the entire inlet plane. Uniform distributions are commonly assumed for simple flows, but any distribution can be specified. Equations $2.23 \mathrm{a}-2.23 \mathrm{~h}$ show a list of the inlet boundary conditions for gas flow. Often, mean values of inlet variables may be known from experimental data; however, the distribution of values of a variable across the inlet is frequently not known from experiment. In this case a uniform or inlet distribution frequently used by other researchers (such as a normal 
distribution for particle number density) is initially used in calculations. Results are checked against experimental results and adjustments are made if necessary.

$$
\begin{aligned}
& u(0, y)=u_{0}(y) \\
& v(0, y)=v_{0}(y) \\
& Y_{f}(0, y)=Y_{f, 0}(y) \\
& Y_{0}(0, y)=Y_{0,0}(y) \\
& Y_{p}(0, y)=Y_{p, 0}(y) \\
& Y_{e}(0, y)=Y_{e, 0}(y) \\
& T(0, y)=T_{0}(y) \\
& \left.\left(\partial^{2} p / \partial x^{2}\right)\right|_{x=0}=0 \text { and } p\left(0, y_{p r e f}\right)=p_{0} \text { (adjusted to satisfy Bernoulli's equation) }
\end{aligned}
$$

For the $\mathrm{i}^{\text {th }}$ lump particles, the inlet boundary conditions are expressed as Eqs. 2.24a-2.24d.

$$
\begin{aligned}
& \mathrm{n}_{\mathrm{i}}(0, \mathrm{y})=\mathrm{n}_{\mathrm{i}, \mathrm{o}}(\mathrm{y}) \\
& \mathrm{u}_{\mathrm{i}}(0, \mathrm{y})=\mathrm{u}_{\mathrm{i}, 0}(\mathrm{y}) \\
& \mathrm{v}_{\mathrm{i}}(0, \mathrm{y})=\mathrm{v}_{\mathrm{i}, 0}(\mathrm{y}) \\
& \mathrm{T}_{\mathrm{i}}(0, \mathrm{y})=\mathrm{T}_{\mathrm{i}, \mathrm{o}}(\mathrm{y})
\end{aligned}
$$

Even worse than the lack of experimental data to set inlet variable distributions, exit boundary conditions are often more difficult to specify because of lack of information on even some of the variable values. Fortunately, in many situations the exit plane can be defined where axial (or $\mathrm{x}$-direction) diffusion is negligible compared to axial convection and the axial flow properties are all nearly smooth (they have a nearly constant and small slope). Under these conditions, a zero slope exit boundary might seem reasonable; however, to ensure excellent convergence in the solution algorithm, the second rather than the first derivative of variables in the $\mathrm{x}$-direction is assumed to be zero, and to account for a small slope at the exit plane, the exit axial velocity distribution is adjusted during a calculation to maintain a global mass balance to near machine precision. Therefore, the exit boundary conditions for the gas flow are

$$
\begin{aligned}
& \left.\left(\partial^{2} \mathrm{u} / \partial \mathrm{x}^{2}\right)\right|_{\mathrm{x}=1}=0 \\
& \left.\left(\partial^{2} \mathrm{v} / \partial \mathrm{x}^{2}\right)\right|_{\mathrm{x}=1}=0 \\
& \left.\left(\partial^{2} \mathrm{Y}_{\mathrm{f}} / \partial \mathrm{x}^{2}\right)\right|_{\mathrm{x}=1}=0 \\
& \left.\left(\partial^{2} \Phi / \partial \mathrm{x}^{2}\right)\right|_{\mathrm{x}=1}=0
\end{aligned}
$$




$$
\begin{aligned}
& \left.\left(\partial^{2} Y_{e} / \partial x^{2}\right)\right|_{x=1}=0 \\
& \left.\left(\partial^{2} p / \partial x^{2}\right)\right|_{x=1}=0 \\
& \left.\left(\partial^{2} h / \partial x^{2}\right)\right|_{x=1}=0
\end{aligned}
$$

For the $\mathrm{i}^{\text {th }}$ size lump of particles, the exit boundary conditions are

$$
\begin{aligned}
& \left.\left(\partial^{2} n_{i} / \partial x^{2}\right)\right|_{x=1}=0 \\
& \left.\left(\partial^{2} u_{i} / \partial x^{2}\right)\right|_{x=1}=0 \\
& \left.\left(\partial^{2} v_{i} / \partial x^{2}\right)\right|_{x=1}=0 \\
& \left.\left(\partial^{2} T_{i} / \partial x^{2}\right)\right|_{x=1}=0
\end{aligned}
$$

The wall is considered to be insulated and impermeable. No-slip conditions are applied to both the gas and particles on the walls. With these assumptions, the gas boundary conditions on the walls are

$$
\begin{aligned}
& u(x, 1 / 2)=u(x,-1 / 2)=0 \\
& v(x, 1 / 2)=v(x,-1 / 2)=0 \\
& \left.\left(\partial Y_{f} / \partial y\right)\right|_{y= \pm 1 / 2}=0 \\
& \left.(\partial \Phi / \partial y)\right|_{y= \pm 1 / 2}=0 \\
& \left.\left(\partial Y_{f} / \partial y\right)\right|_{y= \pm 1 / 2}=0 \\
& \left.(\partial p / \partial y)\right|_{y= \pm 1 / 2}=0 \\
& \left.(\partial h / \partial y)\right|_{y= \pm 1 / 2}=0
\end{aligned}
$$

For the $\mathrm{i}^{\text {th }}$ size lump of particles, the exit boundary conditions are

$$
\begin{aligned}
& \left.\left(\partial n_{i} / \partial y\right)\right|_{y= \pm 1 / 2}=0 \\
& u_{i}(x, 1 / 2)=u_{i}(x,-1 / 2)=0 \\
& v_{i}(x, 1 / 2)=v_{i}(x,-1 / 2)=0 \\
& \left.\left(\partial T_{i} / \partial y\right)\right|_{y= \pm 1 / 2}=0
\end{aligned}
$$


The no-slip boundary at a wall for particles is treated differently for particles than for the gas. In the gas phase, a wall blocking the gas flow (creating a stagnation point) will not cause a problem in the solution procedure in either a physical or numerical sense because pressure will adjust itself upward, forcing the gas to flow around or away from the obstructing wall. In the particle phase under dilute conditions, only the force of drag on the particles by the gas phase is present to push particles away from or around a wall obstruction. Often, depending on particle size and slip velocity, the drag force is not sufficient to prevent particles from colliding with the wall. Under these conditions, the no-slip condition for particles is implemented by calculating particle collision rates with the wall based on particle momentum and drag. Particles colliding with the wall are assumed to leave the flow system and stick to the wall. Under dilute particle loadings, steady-state computations are performed using an assumed quasi-steady wall geometry, meaning that the particle buildup rate on the wall is small compared to other flow and reaction processes in the flow field, so that changes in wall geometry need not be taken into account. Note that, even though the quasi-steady wall geometry assumption is used, particle buildup rates on the wall are computed and can be used to calculate an upper bound on the time to nozzle clogging or buildup times sufficient to adversely affect system performance and require disassembly and clean-out.

In short, 11 governing equations are derived for solving gas flow properties (including an additional variable for richness), and $\mathrm{k}$ sets of governing equations (each has four governing equations), are derived for solving particle flow properties of $\mathrm{k}$ particle size lumps. The gas governing equations include seven partial differential equations, Eqs. 2.1-2.4, 2.6, 2.8, and 2.12, and four algebraic equations, Eqs. 2.5 and 2.9-2.11. The particle governing equations are partial differential equations, Eqs. 2.18-2.21. The flow properties to be solved for are pressure, density, $\mathrm{x}$-velocity, $\mathrm{y}$-velocity, enthalpy, temperature, the richness, and species of fuel, oxidizer, product, and inert for gas, and number density, $\mathrm{x}$-velocity, $\mathrm{y}$-velocity, and temperature for particles. Some source terms of the governing equations, gas species compositions and properties, turbulent viscosity $\mu_{\mathrm{t}}$, and particle diffusivity turbulent viscosity $\Gamma_{\mathrm{n}, \mathrm{i}}$ are yet to be presented and discussed in Chapter 3. The unknown source terms are $S_{1}, S_{u, e}, S_{u, d}, S_{v, e}, S_{v, d}, S_{h, c}, S_{h, d}$, and $S_{Y, f}$ in the gas equations and $S_{n, i}, S_{u, i}, S_{v, i}$, and $S_{T, i}$ in the particle equations. 


\section{PHENOMENOLOGICAL SUBMODELS}

Phenomenological submodels were developed to determine the source terms, diffusivity coefficients and empirical constants needed for the governing equations of the ICOMFLO computer code. The major submodels include an integral reacting flow submodel, a multiphase k- $\varepsilon$ turbulence submodel, an interfacial drag and heat transfer submodel, and a particle melting/evaporation submodel. Other minor submodels include such things as very elaborate data fitting of thermodynamic data for specific applications where the application may be very sensitive to changes in thermodynamic data (for example, as a function of temperature and pressure). The integral reacting flow submodel is one of the most significant and unique features of the ICOMFLO computer code. The new submodel makes numerical calculation of the complex combustion processes in multidimensional flow fast and efficient while still preserving the major physical effects of the combustion on the flow development; in many cases, the submodel eliminates numerical instabilities that render computation to a converged solution with other combustion models impossible. Therefore, the new reaction submodel greatly extends the set of combustion problems for which a converged numerical solution can be obtained.

A new integral reacting flow submodel is developed to define compositions of lumped gas species and their molecular weights $(w)$ and specific heats $(\mathrm{Cp})$ and to determine the source term, $\mathrm{S}_{\mathrm{Y}, \mathrm{f}}$, of the fuel species conservation Eq. 2.6 and the source term, $\mathrm{S}_{\mathrm{h}, \mathrm{c}}$, of the enthalpy equation, Eq. 2.4. Launder and Spalding's popular two-parameter turbulence submodel is modified for the determination of turbulent viscosity $\mu_{\mathrm{t}}$ and particle diffusivity turbulent viscosity $\Gamma_{\mathrm{n}, \mathrm{i}}$ in a twophase flow [19]. An empirical interfacial submodel is used to determine the source term, $S_{u, d}$, of the gas $\mathrm{X}$-momentum Eq. 2.2, the source term, $\mathrm{S}_{\mathrm{v}, \mathrm{d}}$, of the gas $\mathrm{y}$-momentum Eq. 2.3, the source term, $\mathrm{S}_{\mathrm{h}, \mathrm{d}}$, of the enthalpy Eq. 2.4, the source term, $\mathrm{S}_{\mathrm{u}, \mathrm{i}}$, of the particle $\mathrm{x}$-momentum Eq. 2.20, the source term, $\mathrm{S}_{\mathrm{v}, \mathrm{i}}$, of the particle $\mathrm{y}$-momentum Eq. 2.21, and the source term, $\mathrm{S}_{\mathrm{h}, \mathrm{i}}$, of the particle energy Eq. 2.22. The evaporation submodel is used to determine the source term, $S_{1}$, of the gas continuity Eq. 2.1, the source term, $\mathrm{S}_{\mathrm{n}, \mathrm{i}}$, of the particle number density Eq. 2.19, the source term, $\mathrm{S}_{\mathrm{u}, \mathrm{e}}$, of the gas $\mathrm{x}$-momentum Eq. 2.2, and the source term, $\mathrm{S}_{\mathrm{v}, \mathrm{e}}$, of the gas y-momentum Eq. 2.3.

\subsection{Integral Reacting Flow Submodel}

Calculations of combustion computer codes have long been troubled by numerical convergence and instability problems. In a combustion flow, the reaction time scale is generally much smaller than the flow time scale. The difference between the reaction and flow time scales is believed to cause the numerical problems. A conventional combustion flow computer code uses an Arrhenius equation to represent the reaction rate. The Arrhenius reaction rate is expressed in a differential form. When the reaction time scale is much smaller than the flow time scale, the Arrhenius reaction rate becomes extremely large in a combustion flow calculation and often grossly overestimates the mean reaction for many cells in the computational grid. Then, this gross overprediction leads to numerical instability and either nonconverging oscillation or divergence of the computation. An integral lump reacting flow submodel can be developed to replace 
the conventional Arrhenius-type reaction rate submodel. In the integral submodel, it is assumed that the overall reaction progress and its physical effects can be expressed by empirical correlations or tabulated data relating the extent of reaction (or the fraction of fuel consumed) and the accumulated heat of combustion to a flow time scale. The correlations or data tables are derived from the results of detailed kinetics calculations, which provide data to tabulate or correlate an extent of reaction as a function of time. The detailed kinetics calculations can be carried out by a separate kinetic computer code, for example, the National Aeronautics and Space Administration's (NASA's) General Chemical Kinetics Program [20]. An accumulated heat of combustion, which is the heat release rate integrated from the initial time to a specific lag time of the reaction, can also be obtained from the kinetics calculation. A submodel developed for a coal-fired combustor serves as an example to show the steps required to develop an integral reaction submodel for a specific application.

The ICOMFLO computer code has been used to simulate the combustion flow of one of TRW's coal-fired combustors $[21,22]$. The combustor produces a high-temperature plasma flow to generate electricity in an MHD generator. The combustor is a two-stage combustor with a deswirl section connecting the two combustors as shown in Fig. 3. In the first stage, the pulverized coal burns with a substoichiometric amount of preheated air in a swirl motion. The substoichiometric combustion produces a coal-gas with minimal $\mathrm{NO}_{\mathrm{x}}$ generation and the swirl motion removes most of the slag. In the swirl section, the coal-gas flow is seeded with potassium material, and the seeded coal-gas flow turns a 90 -degree angle before entering the second-stage combustor. The seed material is added to make an electrically conducting fluid (plasma) at the flame temperature (about $2700^{\circ} \mathrm{K}$ ), and the flow turn damps the swirl. In the second stage, the seeded coal-gas burns with additional oxidizer to produce a high-temperature plasma flow.

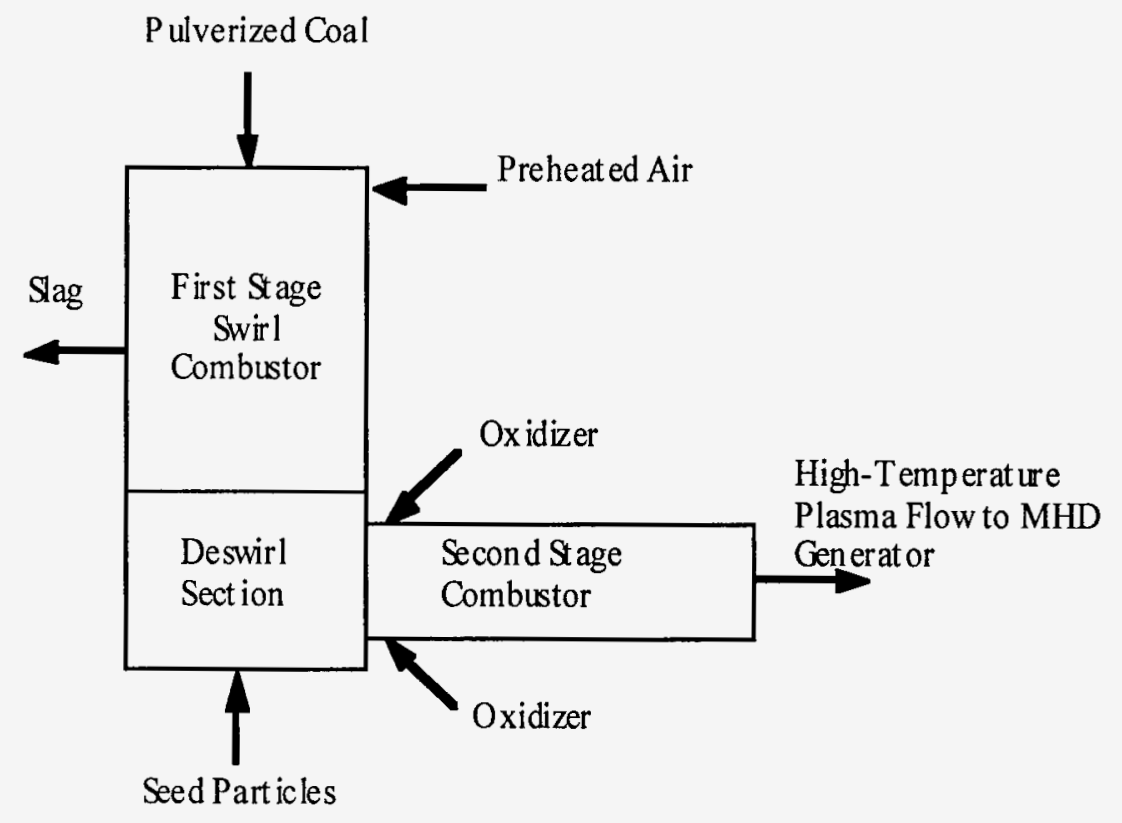

Figure 3 TRW's Coal-Fired MHD Combustor System 
The combustion processes of the second-stage MHD combustor involve 14 species in 30 reactions [16]. Direct applications of these reactions in a CFD calculation can cause severe numerical instability problems. To overcome these problems, an integral lumped reaction submodel is developed. The development of the submodel consists of the following three steps: (1) a four-lump reaction is defined based on the inlet flow conditions, (2) the concentrations of lumped species and cumulative heat of reaction are computed as functions of time, and (3) the time-dependent functions are used to determine the source terms $S_{Y, f}$ and $S_{h, c}$.

A four-lump reaction (A) is assumed for the MHD second-stage combustion. The lumped species are fuel, oxidizer, product, and inert. Fuel species reacts with oxidizer species to produce product species, and inert species is nonreactive.

$$
\text { fuel }+ \text { oxidizer }+ \text { inert } \longrightarrow \text { product }+ \text { inert }
$$

The chemical formula of the lumped species can be derived from the inlet flow compositions. The inl et conditions of the MHD second-stage combustion flow are listed in Table 1. The inlet gas temperature is $1750 \mathrm{~K}$ and the pressure is $5.7 \mathrm{~atm}$. The inlet gas is a mixture of CO (27.2\%), $\mathrm{H}_{2}(8.1 \%), \mathrm{O}_{2}(17.7 \%), \mathrm{CO}_{2}(7.5 \%), \mathrm{H}_{2} \mathrm{O}(10 \%)$, and $\mathrm{N}_{2}(29.5 \%)$. The mixture of $\mathrm{CO}$ and $\mathrm{H}_{2}$ is defined as the fuel $\mathrm{CH}_{0.6} \mathrm{O}$, oxygen is the oxidizer $\mathrm{O}_{1.3}$, the mixture of $\mathrm{CO}_{2}$ and $\mathrm{H}_{2} \mathrm{O}$ is the product $\mathrm{CH}_{0.6} \mathrm{O}_{2.3}$, and $\mathrm{N}_{2}$ is the inert species. The molecular weights of the lumped species are calculated directly (in $\mathrm{kg} / \mathrm{kmol}$ ) from their chemical formulae: 28.6 for fuel, 20.76 for oxidizer, 49.36 for product, and 28 for inert species. The specific heats of the lumped species are estimated (in $\mathrm{kJ} / \mathrm{kmol}-\mathrm{K}$ ) based on the mixture components: 37.6 for fuel, 17.6 for oxidizer, 55.2 for product, and 29.1 for inert species. The chemical formulae, molecular weights, and specific heats of the lumped species are summarized in Table 2.

Table 1 Properties of the Inlet Combustion Flow

\begin{tabular}{|c|c|}
\hline Flow Properties & Property Value \\
\hline Temperature $(\mathrm{K})$ & 1750 \\
\hline Pressure $(\mathrm{Pa})$ & $5.83 \times 10^{5}$ \\
\hline Gas composition (mole fraction) & \\
\hline $\mathrm{CO}$ & 0.272 \\
\hline $\mathrm{H}_{2}$ & 0.081 \\
\hline $\mathrm{O}_{2}$ & 0.177 \\
\hline $\mathrm{CO}_{2}$ & 0.075 \\
\hline $\mathrm{H}_{2} \mathrm{O}$ & 0.100 \\
\hline $\mathrm{N}_{2}$ & 0.295 \\
\hline
\end{tabular}

Table 2 Molecular Weights and Species Heats of the Lumped Species

\begin{tabular}{|l|c|c|}
\hline Lumped Species & $\mathrm{w}(\mathrm{kg} / \mathrm{kmol})$ & $\mathrm{C}_{0}(\mathrm{~J} / \mathrm{kmol}-\mathrm{K})$ \\
\hline Fuel $\left(\mathrm{CH}_{06} \mathrm{O}\right)$ & 28.60 & 37,630 \\
\hline Oxidizer $\left(\mathrm{O}_{13}\right)$ & 20.76 & 17,560 \\
\hline Product $\left(\mathrm{CH}_{06} \mathrm{O}_{23}\right)$ & 49.36 & 55,200 \\
\hline Inert $\left(\mathrm{N}_{2}\right)$ & 28.00 & 29,110 \\
\hline
\end{tabular}


The extent of reaction $\zeta$ is defined as the mass ratio of the fuel consumed to the original fuel. It is an important parameter to represent a combustion process because it decreases monotonically with the reaction time. The extent of reaction for the lump reaction (A) is defined as

$$
\zeta=-\frac{1}{Y_{f, o}} \int_{0}^{t} \frac{d Y_{f}}{d t} d t,
$$

where $t$ is the reaction time and $Y_{f, o}$ is the initial fuel concentration.

A NASA kinetics computer code [19] was used to compute the time-dependent temperature, pressure, density, and species concentrations of the $\mathrm{MHD}$ second-stage combustion. The combustion process involves 14 species (i.e., $\mathrm{H}, \mathrm{OH}, \mathrm{O}, \mathrm{H}_{2}, \mathrm{HO}_{2}, \mathrm{O}_{2}, \mathrm{H}_{2} \mathrm{O}_{2}, \mathrm{H}_{2} \mathrm{O}, \mathrm{CO}, \mathrm{CO}_{2}$, $\mathrm{NO}_{2}, \mathrm{NO}, \mathrm{N}_{2}$, and $\mathrm{N}$ ) in 30 reactions. The rate constants $\mathrm{k}_{\mathrm{f}}$ of these reactions are expressed as

$$
k_{\mathrm{f}}=\mathrm{k}_{\mathrm{o}} \exp \left(-\mathrm{E}_{\mathrm{o}} / \mathrm{RT}\right)
$$

The reactions and their rate constants $\mathrm{k}_{0}, \mathrm{n}$, and $\mathrm{E}_{0}$ recommended by Pitz and Westbrook [23] are listed in Table 3.

\begin{tabular}{|c|c|c|c|c|c|}
\hline \multicolumn{3}{|c|}{ Reactions } & $\mathrm{k}_{0}$ & $\mathrm{n}$ & $\mathrm{E}_{0}$ \\
\hline $\mathrm{H}+\mathrm{OH}$ & $\rightarrow$ & $\overline{\mathrm{O}+\mathrm{H}_{2}}$ & $8.31 \times 10^{9}$ & 1. & 6950. \\
\hline $\mathrm{H}+\mathrm{HO}_{2}$ & $\rightarrow$ & $\mathrm{H}_{2}+\mathrm{O}_{2}$ & $2.51 \times 10^{13}$ & 0. & 695. \\
\hline $\mathrm{H}+\mathrm{HO}_{2}^{2}$ & $\rightarrow$ & $\mathrm{OH}+\mathrm{OH}$ & $2.51 \times 10^{14}$ & 0. & 1887. \\
\hline $\mathrm{H}+\mathrm{H}_{2} \mathrm{O}_{2}$ & $\rightarrow$ & $\mathrm{H}_{2}+\mathrm{HO}_{2}$ & $1.70 \times 10^{12}$ & 0. & 3952. \\
\hline $\mathrm{H}+\mathrm{H}_{2} \mathrm{O}_{2}$ & $\rightarrow$ & $\mathrm{OH}+\mathrm{H}_{2} \mathrm{O}$ & $5.01 \times 10^{14}$ & 0 . & 9929. \\
\hline $\mathrm{O}+\mathrm{OH}$ & $\rightarrow$ & $\mathrm{H}+\mathrm{O}_{2}$ & $6.31 \times 10^{11}$ & 0.5 & 0. \\
\hline $\mathrm{O}+\mathrm{HO}_{2}$ & $\rightarrow$ & $\mathrm{OH}+\mathrm{O}_{2}$ & $5.01 \times 10^{13}$ & 0. & 993. \\
\hline $\mathrm{O}+\mathrm{H}_{2} \mathrm{O}_{2}$ & $\rightarrow$ & $\mathrm{OH}+\mathrm{HO}_{2}$ & $2.00 \times 10^{13}$ & 0. & 5898. \\
\hline $\mathrm{H}_{2}+\mathrm{OH}$ & $\rightarrow$ & $\mathrm{H}+\mathrm{H}_{2} \mathrm{O}$ & $2.19 \times 10^{13}$ & 0. & 5143. \\
\hline $\mathrm{H}_{2}+\mathrm{O}_{2}$ & $\rightarrow$ & $\mathrm{OH}+\mathrm{OH}$ & $1.70 \times 10^{13}$ & 0. & 48150. \\
\hline $\mathrm{OH}+\mathrm{OH}^{2}$ & $\rightarrow$ & $\mathrm{O}+\mathrm{H}_{2} \mathrm{O}$ & $6.31 \times 10^{12}$ & 0. & 1092 . \\
\hline $\mathrm{OH}+\mathrm{HO}_{2}$ & $\rightarrow$ & $\mathrm{O}_{2}+\mathrm{H}_{2} \mathrm{O}$ & $3.02 \times 10^{13}$ & 0. & 0 . \\
\hline $\mathrm{OH}+\mathrm{H}_{2} \mathrm{O}_{2}$ & $\rightarrow$ & $\mathrm{H}_{2} \mathrm{O}+\mathrm{HO}_{2}$ & $1.00 \times 10^{13}$ & 0. & 1807. \\
\hline $\mathrm{HO}_{2}+\mathrm{HO}_{2}$ & $\rightarrow$ & $\mathrm{O}_{2}+\mathrm{H}_{2} \mathrm{O}_{2}$ & $1.00 \times 10^{13}$ & 0. & 993. \\
\hline $\mathrm{H}+\mathrm{H}$ & $\rightarrow$ & $\mathrm{H}_{2}+\mathrm{M}$ & $2.63 \times 10^{15}$ & -1.0 & 0 . \\
\hline $\mathrm{H}+\mathrm{O}$ & $\rightarrow$ & $\mathrm{OH}+\mathrm{M}$ & $1.00 \times 10^{16}$ & 0. & 0. \\
\hline $\mathrm{H}+\mathrm{OH}$ & $\rightarrow$ & $\mathrm{H}_{2} \mathrm{O}+\mathrm{M}$ & $1.41 \times 10^{23}$ & -2 & 0. \\
\hline $\mathrm{H}+\mathrm{O}_{2}$ & $\rightarrow$ & $\mathrm{HO}_{2}+\mathrm{M}$ & $5.01 \times 10^{15}$ & 0. & -993. \\
\hline $\mathrm{O}+\mathrm{O}$ & $\rightarrow$ & $\mathrm{O}_{2}+\mathrm{M}$ & $1.00 \times 10^{14}$ & 0. & -1430 \\
\hline $\mathrm{OH}+\mathrm{OH}$ & $\rightarrow$ & $\mathrm{H}_{2} \mathrm{O}_{2}+\mathrm{M}$ & $9.12 \times 10^{14}$ & 0. & 4468. \\
\hline $\mathrm{CO}+\mathrm{OH}$ & $\rightarrow$ & $\mathrm{CO}_{2}+\mathrm{H}$ & $1.51 \times 10^{7}$ & 1.3 & -.77 \\
\hline $\mathrm{CO}+\mathrm{HO}_{2}$ & $\rightarrow$ & $\mathrm{CO}_{2}+\mathrm{OH}$ & $1.51 \times 10^{14}$ & 0. & 23.65 \\
\hline $\mathrm{CO}+\mathrm{O}$ & $\rightarrow$ & $\mathrm{CO}_{2}+\mathrm{M}$ & $5.89 \times 10^{15}$ & 0. & 4.1 \\
\hline $\mathrm{CO}_{2}+\mathrm{O}$ & $\rightarrow$ & $\mathrm{CO}+\mathrm{O}_{2}$ & $2.75 \times 10^{12}$ & 0. & 43.83 \\
\hline $\mathrm{M}+\mathrm{NO}_{2}$ & $\rightarrow$ & $\mathrm{NO}+\mathrm{O}^{2}$ & $1.1 \times 10^{16}$ & 0. & 65000. \\
\hline $\mathrm{O}+\mathrm{N}_{2}$ & $\rightarrow$ & $\mathrm{NO}+\mathrm{N}$ & $7.6 \times 10^{13}$ & 0. & 75510. \\
\hline $\mathrm{N}+\mathrm{O}_{2}$ & $\rightarrow$ & $\mathrm{NO}+\mathrm{O}$ & $6.4 \times 10^{9}$ & 1.0 & 6260. \\
\hline $\mathrm{NO}_{2}+\mathrm{H}$ & $\rightarrow$ & $\mathrm{NO}+\mathrm{OH}$ & $3.5 \times 10^{14}$ & 0. & 900. \\
\hline $\mathrm{NO}_{2}+\mathrm{O}$ & $\rightarrow$ & $\mathrm{NO}+\mathrm{O}_{2}$ & $1.0 \times 10^{13}$ & 0. & 600. \\
\hline $\mathrm{O}_{2}$ & & $\mathrm{NO}_{2}+\mathrm{OH}$ & $1.2 \times 10^{13}$ & & 2400. \\
\hline
\end{tabular}

Table 3 Reactions and Rate Constants of the MHD Combustion Flow 


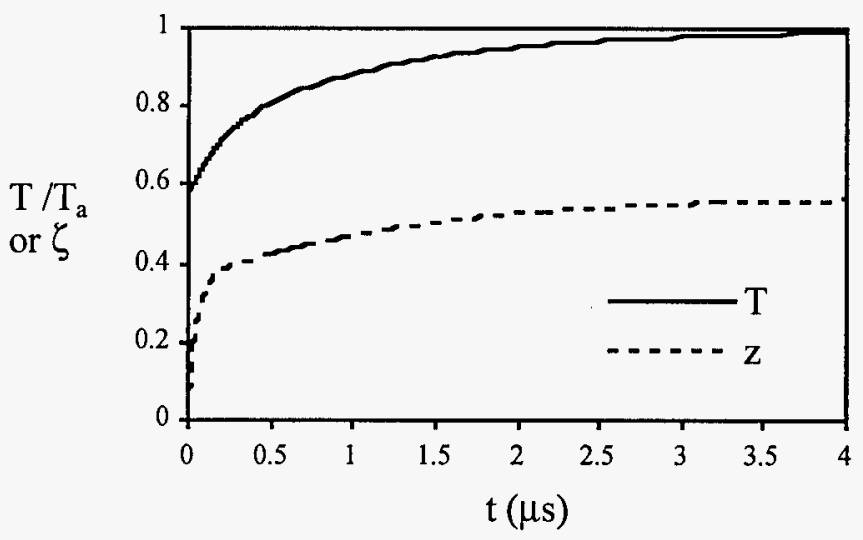

Figure 4 Progress of Reaction Derived from Kinetics Calculation

The four lumped species can be defined from the 16 combustion species. Among the combustion species, $\mathrm{CO}, \mathrm{H}_{2}, \mathrm{O}_{2}, \mathrm{CO}_{2}, \mathrm{H}_{2} \mathrm{O}$, and $\mathrm{N}_{2}$ have been defined for the lumped species. The other 10 combustion species need to be assigned to the lumped species according to their atomic elements. The $\mathrm{H}$ atoms are assigned to the fuel species; the $\mathrm{O}$ atoms are assigned to the oxidizer species; and the $\mathrm{N}$ atoms are assigned to the inert species. Therefore, time-dependent combustion species concentrations computed from the kinetics code can be converted to the lumped species concentrations, from which a time-dependent extent of reaction can be determined.

Figure 4 plots the computed time-dependent temperature (solid line) and extent of reaction (dashed line). The temperature value in the figure is normalized by an adiabatic flame temperature, $\mathrm{T}_{\mathrm{a}}$, of $3000^{\circ} \mathrm{K}$. The results show that temperature increases from 1750 to $2985^{\circ} \mathrm{K}$ in about $4 \mu \mathrm{s}$, and in the same time the extent of reaction increases from 0 to 0.57 . At the end, $43 \%$ of the fuel remains because of the high-temperature dissociation.

The computed extent of reaction can also be correlated as a function of reaction time:

$$
\begin{array}{llc}
\zeta & =0.1495 \ln (80 \mathrm{t}+1) & \mathrm{t} \leq 0.1 \mu \mathrm{s} \\
\zeta & =1-\exp \left(-0.705 \mathrm{t}^{0.25}\right) & 0.1<\mathrm{t} \leq 0.25 \mu \mathrm{s} \\
\zeta & =0.57-0.1774 \exp \left[-0.78(\mathrm{t}-0.25)^{1.04}\right] & \mathrm{t}>0.25 \mu \mathrm{s}
\end{array}
$$

where reaction time is expressed in $\mu$ s. Similarly, accumulated heat of combustion can be computed from the detailed kinetics computational results and correlated as a function of the extent of reaction. The correlation for accumulated heat of combustion is:

$$
\begin{array}{ll}
\mathrm{H}_{\mathrm{f}}=0.001 \exp (9.52 \zeta) & \zeta \leq 0.393 \\
\mathrm{H}_{\mathrm{f}}=56.9-\exp (0.57-\zeta) & \zeta>0.393
\end{array}
$$

The reaction time scale of about $4 \mu \mathrm{s}$ is much smaller than the flow time scale for a typical computational grid cell, which is on the order of milliseconds in this case. The big difference between these two time scales has caused severe numerical problems in the previously employed differential reaction submodel. To prevent this problem from occurring, the new reaction flow 
submodel expresses heat release and fuel consumption in a flow time scale by integrating over the reaction time scale. In the new computer code, the time-resolved extent of reaction is formulated as a sink term for the fuel species equation. The sink term is written as

$$
S_{Y, f}=-\rho Y_{f} V_{c} \frac{d \zeta}{d L_{c}}
$$

where $\mathrm{V}_{c}$ is a convective velocity scale and $\mathrm{L}_{c}$ is a convective length scale. The convective velocity and length scales are determined from the computed flow field and the grid point assignment. As for the heat of combustion, the source term (including the empirical accumulated heat of combustion) is formulated for the enthalpy equation. The source term is written as

$$
S_{h, c}=\frac{\rho V_{c}}{w_{f}} \frac{d H_{f}}{d \zeta} \frac{d \zeta}{d L_{c}}
$$

\subsection{Multiphase k- $\varepsilon$ Turbulence Submodel}

A turbulent flow consists of a spectrum of rotational eddies. The eddies, ranging in size from a tiny molecular viscous motion to the size of the flow, can effectively transport and mix species, momentum, and heat of the flow. The mixing rates of the turbulent motion are generally several orders of magnitude greater than those of the molecular diffusion. In modeling the turbulence, eddies are generally divided into two size groups: large and small eddies. Large eddies transport flow species, momentum, and heat from the mean flow and eventually degenerate into small eddies; small eddies dissipate the turbulent kinetic energy through turbulent viscosity. Turbulent kinetic energy is defined as $\mathrm{k}=\mathrm{u}^{\prime 2} / 2$, where $\mathrm{u}^{\prime}$ is the velocity fluctuation, and turbulent dissipation rate is called $\varepsilon$. Launder and Spalding first developed a turbulent model employing the turbulent quantities $\mathrm{k}$ and $\varepsilon$ for single-phase flows [19], and Zhou and Chiu later modified the turbulence model for multiphase flows [2] into the one used in the ICOMFLO code.

Launder and Spalding assumed that the turbulent diffusivity $\mu_{t}$ is proportional to gas density $\rho$, velocity fluctuation $u^{\prime}$, and a macro turbulent length scale $\lambda_{t}$ as shown in Eq. 3.7, in which $\mathrm{C}_{\mu}$ is the proportionality constant.

$$
\mu_{\mathrm{t}}=\mathrm{C}_{\mu} \rho u^{\prime} \lambda_{\mathrm{t}} / 2
$$

In Eq. 3.7, $u^{\prime}$ and $\lambda_{t}$ are two unknown flow properties to be determined in the solution procedure. For an eddy size $\lambda_{t}$, the turbulent time scale is $\lambda_{t} / u^{\prime}$. Large eddies are assumed to lose their kinetic energy to small eddies for dissipation within one "turnover" time $\lambda_{t} / u^{\prime}$. Therefore, the rate of dissipation is equal to the transport rate of turbulent kinetic energy as shown in Eq. 3.8.

$$
\varepsilon=\mathrm{k}\left(\mathrm{u}^{\prime} / \lambda_{\mathrm{t}}\right)
$$

From Eq. 3.8, the macro turbulent length scale can be expressed as 


$$
\lambda_{\mathrm{t}}=\mathrm{k}\left(\mathrm{u}^{\prime} / \varepsilon\right)
$$

Substituting Eq. 3.9 into Eq. 3.7, an alternative turbulent diffusivity expression is obtained as

$$
\mu_{\mathrm{t}}=\mathrm{C}_{\mu} \rho \mathrm{k}^{2} / \varepsilon
$$

In the turbulent diffusivity Eq. 3.10, there are still two unknown flow properties $\mathrm{k}$ and $\varepsilon$. Launder and Spalding proposed to determine the two unknowns by solving two additional transport equations. Equations 3.11 and 3.12 are derived for the turbulence quantities $\mathrm{k}$ and $\varepsilon$, respectively. These equations are to be solved simultaneously with the governing equations of a single-phase flow. The source terms of the k-equation imply that turbulent kinetic energy is generated by the viscous dissipation of the mean velocity arising out of velocity gradients in the flow field, while it is dissipated by turbulence fluctuations at a rate $\varepsilon$. The source terms of the $\varepsilon$ equation are similar to the two terms in the k-equation, except that they are multiplied by a factor $(\varepsilon / k)$ and a pair of empirical constants, $\mathrm{C}_{1}$ and $\mathrm{C}_{2}$.

Turbulence transport equation of $\mathrm{k}$ (single phase):

$$
\frac{\partial}{\partial x}\left(\rho u k-\frac{\mu_{t}}{\sigma_{k}} \frac{\partial k}{\partial x}\right)+\frac{\partial}{\partial y}\left(\rho v k-\frac{\mu_{t}}{\sigma_{k}} \frac{\partial k}{\partial y}\right)=\mu_{t} G_{\mu}-\rho \varepsilon
$$

Turbulence transport equation of $\varepsilon$ (single phase):

$$
\frac{\partial}{\partial x}\left(\rho u \varepsilon-\frac{\mu_{t}}{\sigma_{\varepsilon}} \frac{\partial \varepsilon}{\partial x}\right)+\frac{\partial}{\partial y}\left(\rho v \varepsilon-\frac{\mu_{t}}{\sigma_{\varepsilon}} \frac{\partial \varepsilon}{\partial y}\right)=C_{1} \mu_{t} \frac{\varepsilon}{k} G_{\mu}-C_{2} \rho \frac{\varepsilon^{2}}{k}
$$

Equations 3.10, 3.11, and 3.12 constitute a turbulence model for a single-phase flow. In this model, there are five empirical constants, $\mathrm{C}_{\mu}, \mathrm{C}_{1}, \mathrm{C}_{2}, \sigma_{\mathrm{k}}$ and $\sigma_{\varepsilon}$. The values of these empirical constants recommended by Launder and Spalding [19] are listed below:

$\begin{array}{ccc}\mathrm{C}_{\mu} & 0.09 \\ \mathrm{C}_{1} & 1.44 \\ \mathrm{C}_{2} & 1.92 \\ \sigma_{\mathrm{k}} & 1.0 \\ \sigma_{\varepsilon} & 1.3\end{array}$

On the basis of the work of Ward et al. [24], Zhou and Chiu modified the k- $\varepsilon$ model for multiphase flows to account for turbulent dispersion of particles. The multiphase turbulent transport equations are derived from the single-phase turbulence transport equations, Eqs. 3.11 and 3.12 , by adding a void fraction to the density terms and a sink term in the k-equation, as shown in Eqs. 3.13 and 3.14.

Turbulence transport equation of $\mathrm{k}$ (multiphase):

$$
\frac{\partial}{\partial x}\left(\theta \rho u k-\frac{\mu_{t}}{\sigma_{k}} \frac{\partial k}{\partial x}\right)+\frac{\partial}{\partial y}\left(\theta \rho v k-\frac{\mu_{t}}{\sigma_{k}} \frac{\partial k}{\partial y}\right)=\theta \rho \mu_{t} G_{\mu}-\theta \rho \varepsilon-S_{k, s}
$$


Turbulence transport equation of $\varepsilon$ (multiphase):

$$
\frac{\partial}{\partial \mathrm{x}}\left(\theta \rho u \varepsilon-\frac{\mu_{\mathrm{t}}}{\sigma_{\varepsilon}} \frac{\partial \varepsilon}{\partial \mathrm{x}}\right)+\frac{\partial}{\partial \mathrm{y}}\left(\theta \rho v \varepsilon-\frac{\mu_{\mathrm{t}}}{\sigma_{\varepsilon}} \frac{\partial \varepsilon}{\partial \mathrm{y}}\right)=\mathrm{C}_{1} \mu_{\mathrm{t}} \frac{\varepsilon}{\mathrm{k}} \mathrm{G}_{\mu}-\mathrm{C}_{2} \theta \rho \frac{\varepsilon^{2}}{\mathrm{k}}
$$

In the modification, a sink term is added to the k-equation to account for the loss rate of turbulent kinetic energy in the gas phase as this energy is transferred to particles in the turbulent dispersion process. The loss of the turbulent kinetic energy depends on particle size. If the particle size is in a submicron range, the particles can dissipate the turbulence kinetic energy as effectively as the gas and the loss rate is set to the gas-phase kinetic energy convective rate. If the particle size is larger than $100 \mu \mathrm{m}$, the loss becomes negligible. The added sink term is expressed as

$$
\mathrm{S}_{\mathrm{k}, \mathrm{s}}=\int_{0}^{\infty} \frac{4}{3} \pi \mathrm{r}^{3}\left[\frac{\partial}{\partial \mathrm{x}}\left(\mathrm{gu}_{\mathrm{s}} \mathrm{k} / \varphi^{2}\right)+\frac{\partial}{\partial \mathrm{y}}\left(\mathrm{gv}_{\mathrm{s}} \mathrm{k} / \varphi^{2}\right)\right] \mathrm{dr},
$$

where an empirical particle dispersion function $\varphi$ is defined as

$$
\varphi(r)=1+2 r+0.06 r^{2}
$$

The turbulence quantities $\mathrm{k}$ and $\varepsilon$ are treated as flow properties. Their transport equations (3.13) and (3.14) are elliptic-type partial differential equations. Boundary conditions are needed to solve the transport equations. The boundary conditions are defined as

$$
\begin{aligned}
& k(0, y)=k_{0}(y) \\
& \left.\left(\partial^{2} \varepsilon / \partial x^{2}\right)\right|_{x=0}=0 \\
& \left.\left(\partial^{2} k / \partial x^{2}\right)\right|_{x=1}=0 \\
& \left.\left(\partial^{2} \varepsilon / \partial x^{2}\right)\right|_{x=1}=0 \\
& \left.(\partial k / \partial y)\right|_{y= \pm 1 / 2}=0 \\
& \left.(\partial \varepsilon / \partial y)\right|_{y= \pm 1 / 2}=0
\end{aligned}
$$

Particle diffusivity also depends on particle size. For particles larger than $100 \mu \mathrm{m}$, turbulent fluctuations (eddies) have little effect on them and their momentum is only affected by drag computed from the mean gas flow. If the particle size is in a submicron range, the particles move along with gas flow and have a turbulent diffusion effect similar to that of the gas phase. For intermediate particle sizes (from submicron to $100 \mu \mathrm{m}$ ), the turbulent diffusivity of the particle number density equation (2.18) is defined as

$$
\Gamma_{\mathrm{n}, \mathrm{i}}=\mu_{\mathrm{t}} /(0.9 \varphi \rho)
$$




\subsection{Interfacial Drag and Heat Transfer Submodel}

A gas flow is generally driven by a pressure gradient and a particle flow is driven by the drag force from the gas flow. The drag force is caused by the velocity difference between the gas and solid phases (called slip velocity). A slip velocity $\mathrm{u}_{\delta}$ is defined in Eq. 3.18:

$$
\mathrm{u}_{\delta}=\mathrm{u}-\mathrm{u}_{\mathrm{s}}
$$

Empirical equations are commonly used to correlate the drag force with the slip velocity. Drag force is a vector defined by two orthogonal components: one in the $\mathrm{x}$-direction and the other in the y-direction. Since the derivation of the two components is parallel, only the derivation of the $\mathrm{x}$-component will be presented in the following. For a single particle in the gas, the $\mathrm{x}$ direction drag force $\left(\mathrm{F}_{\mathrm{d}, \mathrm{x}}\right)$ of gas exerted on the particle can be expressed as a function of the slip velocity kinetic energy $\rho u_{\delta}{ }^{2} / 2$, particle cross-sectional area $\pi r^{2}$, and an empirical coefficient $C_{d}$ as shown in Eq. 3.19. The particle drag force is in the same direction as the slip velocity: when the slip velocity is positive, the drag force is positive; when the slip velocity is negative, the drag force is negative.

$$
\mathrm{F}_{\mathrm{d}, \mathrm{x}}=\frac{1}{2} \rho\left|\mathrm{u}_{\delta}\right| \mathrm{u}_{\delta} \pi \mathrm{r}^{2} \mathrm{C}_{\mathrm{d}}
$$

Equation 3.20 is a common expression of the drag coefficient $\mathrm{C}_{\mathrm{d}}$. The coefficient includes two major effects on the drag force; one is a viscous (or diffusive) effect represented by a Reynolds number $\mathrm{Re}_{s}$, Eq. 3.21, and the other is the evaporation effect represented by a transfer number B, Eq. 3.22. In Eq. 3.22, $\mathrm{L}$ is the latent heat of vaporization after melting of a particle and $T_{b}$ is the boiling temperature of a particle's material. When particle temperature is less than its boiling temperature, there is no evaporation, the transfer number is zero, and the drag force depends on the viscous effect alone.

$$
\mathrm{C}_{\mathrm{d}}=\frac{24}{\operatorname{Re}_{\mathrm{s}}} \frac{\left(1+0.15 \mathrm{Re}_{\mathrm{s}}^{0.687}\right)}{1+\mathrm{B}},
$$

where

$$
\begin{aligned}
& \operatorname{Re}_{\mathrm{s}}=\frac{2 \rho\left|\mathrm{u}_{\delta}\right| \mathrm{r}}{\mu} \\
& \mathrm{B}=\left\{\begin{array}{cl}
\frac{\mathrm{C}_{\mathrm{p}}\left(\mathrm{T}_{\mathrm{s}}-\mathrm{T}_{\mathrm{b}}\right)}{\mathrm{L}_{\mathrm{L}}} & \text { if } \mathrm{T}_{\mathrm{s}}>\mathrm{T}_{\mathrm{b}} \\
0 & \text { if } \mathrm{T}_{\mathrm{s}} \leq \mathrm{T}_{\mathrm{b}}
\end{array}\right.
\end{aligned}
$$

By neglecting the collisions between particles, the drag force of the gas on a group of particles can be obtained by adding up the drag forces of single particles. Integrating over particle size space from $r=r_{i-1 / 2}$ to $r_{i+1 / 2}$, the drag force exerted by the gas on the particles of the $i^{\text {th }}$ size lump is shown in Eq. 3.23. The drag force is the source term $S_{u, i}$ of the particle $x$-momentum Eq. 2.20 . 


$$
\mathrm{S}_{\mathrm{u}, \mathrm{i}}=\int_{\mathrm{r}, \mathrm{i}-1 / 2}^{\mathrm{r}, \mathrm{i}+\mathrm{2}} \mathrm{F}_{\mathrm{d}, \mathrm{x}} \mathrm{g}_{\mathrm{s}} \mathrm{dr}=\mathrm{C}_{\mathrm{d}, \mathrm{i}} \rho\left|\mathrm{u}_{\delta, \mathrm{i}}\right| \mathrm{u}_{\delta, \mathrm{i}} \frac{\pi \mathrm{r}_{\mathrm{i}}^{2}}{2} \mathrm{n}_{\mathrm{i}}
$$

The interfacial drag forces of the particles and the gas are conserved if the friction dissipation on particle surface is negligible. The drag force on the gas should be balanced by the drag force on the particles. Integrating over the entire particle size space, one obtains the drag force exerted by the particles on the gas, Eq. 3.24. The drag force on the gas is the source term $S_{u, d}$ for the gas momentum equation, Eq. 2.2.

$$
\mathrm{S}_{\mathrm{u}, \mathrm{d}}=-\int_{0}^{\infty} \mathrm{C}_{\mathrm{d}} \rho\left|\mathrm{u}_{\delta}\right| \mathrm{u}_{\delta} \frac{\pi \mathrm{r}^{2}}{2} \mathrm{~g}_{\mathrm{s}} \mathrm{dr}
$$

In a similar way, source terms of the $y$-direction drag force can be derived. Equation 3.26 is the source term $S_{v, i}$ for the particle momentum Eq. 2.21. Equation 3.25 is the source term $S_{v, d}$ for the gas momentum Eq. 2.3.

$$
\begin{aligned}
& \mathrm{S}_{\mathrm{v}, \mathrm{i}}=\mathrm{C}_{\mathrm{d}, \mathrm{i}} \rho\left|\mathrm{v}_{\delta, \mathrm{i}}\right| \mathrm{v}_{\delta, \mathrm{i}} \frac{\pi \mathrm{r}_{\mathrm{i}}^{2}}{2} \mathrm{n}_{\mathrm{i}} \\
& \mathrm{S}_{\mathrm{v}, \mathrm{d}}=-\int_{0}^{\infty} \mathrm{C}_{\mathrm{d}} \rho\left|\mathrm{v}_{\delta}\right| \mathrm{v}_{\delta} \frac{\pi \mathrm{r}^{2}}{2} \mathrm{~g}_{\mathrm{s}} \mathrm{dr}
\end{aligned}
$$

Again, empirical equations are used to correlate the interfacial heat transfer with the slip velocity. For a single particle in the gas, the heat transfer between the gas and the particle is expressed as

$$
\mathrm{q}_{\mathrm{s}}=2 \pi \mathrm{r} \lambda \mathrm{Nu}_{\mathrm{s}}\left(\mathrm{T}-\mathrm{T}_{\mathrm{s}}\right) \delta\left(\mathrm{T}_{\mathrm{b}}\right)
$$

In Eq. $3.29, \lambda$ is thermal conductivity of the gas, $\mathrm{Nu}_{\mathrm{s}}$ is an empirical formula for a Nusselt number, and $\delta\left(\mathrm{T}_{\mathrm{b}}\right)$ is a delta function (equal to one if particle temperature is less than $\mathrm{T}_{\mathrm{b}}$ and zero otherwise). The Nusselt formula, Eq. 3.27, includes two major effects of the interfacial heat exchange. One is the momentum effect represented by the Reynolds number $\mathrm{Re}_{\mathrm{s}}$, Eq. 3.21, and the other is the diffusivity effect represented by the Schmidt number Sc, Eq. 3.29. In Eq. 3.29, D is the mass diffusivity of a species. After the particle temperature reaches the boiling temperature, further heat transfer from the gas to particles goes into particle evaporation. In this case, the step function $\delta\left(T_{b}\right)$ makes $q_{s}$ equal to zero when $T_{s}$ equals $T_{b}$.

$$
\begin{aligned}
& \mathrm{Nu}_{\mathrm{s}}=1+0.276 \mathrm{Re}_{\mathrm{s}}^{1 / 2} \mathrm{Sc}^{1 / 3} \\
& \mathrm{Sc}=\frac{\mu}{\rho \mathrm{D}}
\end{aligned}
$$

By neglecting the interactions between particles, the heat transfer from the gas to a group of particles can be obtained by adding up the interfacial heat fluxes on these particles. Equation 3.30 shows the interfacial heat transfer from the gas to the particles of the $\mathrm{i}^{\text {th }}$ lump. The interfacial heat transfer is the source term of the particle energy equation.

$$
\mathrm{S}_{\mathrm{T}, \mathrm{i}}=2 \pi \mathrm{r}_{\mathrm{i}} \lambda N \mathrm{Nu}_{\mathrm{s}}\left(\mathrm{T}-\mathrm{T}_{\mathrm{s}, \mathrm{i}}\right) \delta\left(\mathrm{T}_{\mathrm{b}}\right) \mathrm{n}_{\mathrm{i}}
$$


The interfacial heat transfer between the particles and the gas is conserved if there is no heat loss through another medium. The heat transfer from the particles to the gas should be balanced by the heat transfer from the gas to the particles. Integrating over the entire particle size space, the heat transfer from the particles to the gas is shown as Eq. 3.26, which is the source term $\mathrm{S}_{\mathrm{h}, \mathrm{d}}$ for the gas enthalpy, Eq. 2.4.

$$
\mathrm{S}_{\mathrm{h}, \mathrm{d}}=-\int_{0}^{\infty} 2 \pi \mathrm{r} \lambda \mathrm{Nu}_{\mathrm{s}}\left(\mathrm{T}-\mathrm{T}_{\mathrm{s}}\right) \delta\left(\mathrm{T}_{\mathrm{b}}\right) \mathrm{g}_{\mathrm{s}} \mathrm{dr}
$$

\subsection{Particle Evaporation Submodel}

The source term in the gas continuity equation, Eq. 2.1, is the evaporation rate of the particles per unit volume. Neglecting the interactions between the particles, the evaporation rate can be derived from a single particle evaporation theory. The classical solution giving the particle evaporation rate for a single stationary particle is [25]

$$
\left(\frac{\mathrm{dm}}{\mathrm{dt}}\right)_{\text {stat }}=4 \pi \mathrm{r}\left(\lambda / \mathrm{C}_{\mathrm{p}}\right) \ln (1+\mathrm{B})
$$

A correction for convection effect, sometimes referred to as the Ranz-Marshall model [26], can be applied to the stationary solution (Eq. 3.32) to yield an empirical correlation for vaporization in a flow. The correction adds a Nusselt number $\mathrm{Nu}_{\mathrm{s}}$ to the stationary solution. Note that some effects of other evaporating particles in the vicinity of a particle are taken into account indirectly through these formulas and correlations because the other nearby evaporating melted particles change the gas composition and properties, such as $\lambda$ and $C_{p}$.

$$
\left(\frac{\mathrm{dm}}{\mathrm{dt}}\right)_{\mathrm{conv}}=4 \pi \mathrm{r}\left(\lambda / \mathrm{C}_{\mathrm{p}}\right) \ln (1+\mathrm{B}) \mathrm{Nu}_{\mathrm{s}}
$$

The total evaporation rate at a point per unit volume of physical space is determined by integrating the product of single-particle evaporation rate and the spray distribution function over particle size space,

$$
\mathrm{S}_{1}=\int_{0}^{\infty} 4 \pi \mathrm{r}\left(\lambda / \mathrm{C}_{\mathrm{p}}\right) \ln (1+\mathrm{B}) \mathrm{Nu}_{\mathrm{s}} \mathrm{g}_{\mathrm{s}} \mathrm{dr}
$$

Equation 3.34 is hard to discretize because the integrand contains the transfer number $\mathrm{B}$ and the Nusselt number $\mathrm{Nu}$, which are implicit functions of the particle radius, $r$. Using the method of integration by parts, Eq. 3.34 can be converted to Eq. 3.35, which is easier to use in the discretization for the evaporation source term of the particle number density equation, Eq. 2.19.

$$
\mathrm{S}_{1}=-\int_{0}^{\infty} \frac{4}{3} \pi \mathrm{r}^{3}\left(\lambda / \mathrm{C}_{\mathrm{p}}\right) \frac{\partial}{\partial \mathrm{r}}\left[\frac{\ln (1+\mathrm{B}) \mathrm{Nu}_{\mathrm{s}} \mathrm{g}_{\mathrm{s}}}{\mathrm{r}}\right] \mathrm{dr}
$$

Based on Eq. 3.35, the change of particle number density due to evaporation can be derived for each particle group. For the particles of the $\mathrm{i}^{\text {th }}$ lump, the sink term of the particle number density equation is 


$$
S_{n, i}=-\int_{n_{i-1 / 2}}^{n_{i+1 / 2}} \frac{4}{3} \pi r^{3}\left(\lambda / C_{p}\right) \frac{\partial}{\partial r}\left[\frac{\ln (1+B) N u_{s} g_{s}}{r}\right] d r
$$

The particle evaporation also changes the momentum of gas flow. Particle momentum is assumed to be maintained as the particles change phase. Therefore, as particles vaporize, the original particle momentum should be added to the gas flow along with its vaporized mass. The source term $\mathrm{S}_{\mathrm{u}, \mathrm{e}}$ of the $\mathrm{x}$-momentum equation can be formulated as

$$
S_{u, e}=-\int_{0}^{\infty} \frac{4}{3} \pi r^{3}(\lambda / C p) \frac{\partial}{\partial r}\left[\frac{\ln (1+B) N u_{s} g}{r}\right] u_{s} d r
$$

Similarly, the source term $S_{v, e}$ of the $y$-momentum equation is

$$
\mathrm{S}_{\mathrm{v}, \mathrm{e}}=-\int_{0}^{\infty} \frac{4}{3} \pi \mathrm{r}^{3}(\lambda / \mathrm{Cp}) \frac{\partial}{\partial \mathrm{r}}\left[\frac{\ln (1+\mathrm{B}) \mathrm{Nu}_{\mathrm{s}} \mathrm{g}}{\mathrm{r}}\right] \mathrm{v}_{\mathrm{s}} \mathrm{dr}
$$

In short, an integral reaction submodel, a multiphase two-parameter turbulence submodel, an interfacial drag and heat transfer submodel, and a particle evaporation submodel have been developed. The integral reaction submodel defines a lumped reaction (A) for a flow of four lumped species. Molecular weights and specific heats of the lumped species are given in Table 2. Equations of reaction rate (3.3) and accumulated heat release (3.4) were derived from detailed kinetics calculations. The equations are used to define the source term $S_{Y, f}$, of the fuel species conservation equation in Eq. 3.5 and the source term $S_{h, c}$ for the enthalpy equation in Eq. 3.6. The turbulence submodel defines a partial differential equation, Eq. 3.13, for turbulent kinetic energy $\mathrm{k}$ and another partial differential equation, Eq. 3.14, for turbulent dissipation rate $\varepsilon$. Solutions of the two turbulent equations are used to determine the turbulent viscosity $\mu_{\mathrm{t}}$ in Eq. 3.10 and the particle diffusivity turbulence viscosity $\Gamma_{n, i}$ in Eq. 3.18. The interfacial submodel defines empirical drag and heat transfer correlations. The drag force correlation is used to define the source terms $S_{u, i}$ and $S_{v, i}$ of the particle momentum equations in Eqs. 3.23 and 3.25, and the source terms $S_{u, d}$ and $S_{v, d}$ of the gas momentum equations in Eqs. 3.24 and 3.26. The heat transfer correlation is used to define the source term $\mathrm{S}_{\mathrm{T}, \mathrm{i}}$ of the particle energy equation in Eq. 3.30, and the source term $S_{h, d}$ of the gas enthalpy equations in Eq. 3.31. The evaporation submodel derived the particle evaporation rate from a classical theory and expanded it with a well-known correlation to account for convective effects on evaporation.. The evaporation rate is used to define the evaporation source term $S_{1}$ of the particle number density equation in Eq. 3.35, the sink term $S_{n, i}$ of the particle number density equation in Eq. 3.36, and the source terms $\mathrm{S}_{\mathrm{u}, \mathrm{e}}$ and $\mathrm{S}_{\mathrm{uve}}$ of the gas momentum equation in Eqs. 3.37 and 3.38. 


\section{NUMERICAL SCHEME}

Governing equations for gas and particle phases have been derived in chapters 2 and 3 . These equations need to be solved to obtain property values of the flow field. Some equations, i.e., Eqs. 2.1-2.4, 2.6, 2.8, 2.12, 3.13, and 3.14 for gas, and Eqs. 2.18-2.21 for particles, are nonlinear partial differential equations. The differential equations have no general analytical solution and must be solved using numerical methods. A control volume approach is used here. The control volume approach converts the governing equations of a continuous domain to the algebraic equations of a discretized grid system and solves the algebraic equations iteratively.

\subsection{Computational Cells and Staggered Grids}

A grid is a collection of computational cells (or nodes) that cover a flow domain of interest. The ICOMFLO code uses a two-dimensional grid defined in either a Cartesian or a cylindrical coordinate system. In the following discussion, only the Cartesian coordinate system is used.

A cell represents a control volume of a small portion of a flow field in the physical space. The cell can be a boundary or an interior cell. Flow properties of a boundary cell are mostly given, whereas those of an interior cell have to be determined from the governing equations. To solve the equations, a grid system needs to be defined. Consider an interior cell " $p$ " as shown in Fig. 5. Cell "p" (with grid point shown as a solid circle in the middle) is located at the center of the rectangular control volume (shaded area) for scalar cells. The cell has four adjacent cells (with centers at the solid circles on the outside), four interfaces (hollow circles), and four interface corners. The labeling for the cell "p" is taken from map drawing conventions for the direction north, south, east, and west. The adjacent cells are labeled with the capital letters "E," "W," "N," and "S" respectively for the cell to the east, west, north, or south of cell "p." Points on the cell interfaces are labeled with the same convention using lower case letters "e," "w," "n," and "s." The points at northwest, southwest, northeast, and southeast corners are labeled "nw," "sw," "ne," and "se," respectively.

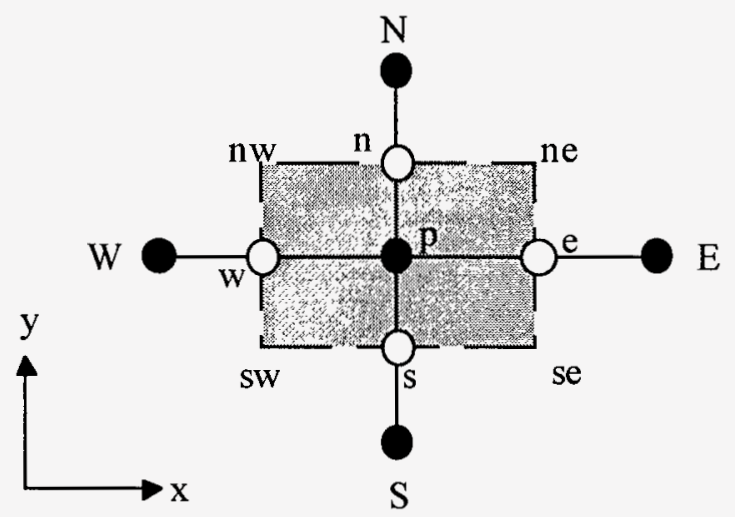

Figure 5 Labeling of a Computational Cell 
An x-y Cartesian coordinate system may be used to define the locations of cell points. The $\mathrm{x}$ (or axial) axis is in the east-west direction and the $\mathrm{y}$ (or transverse) axis is in the north-south direction. The coordinates of the cell points "p," "E," "W," "N," "S," "e," "w," "s," "n," "ne," "nw," "se," and "sw" are denoted as $\left(x_{p}, y_{p}\right),\left(x_{E}, y_{E}\right),\left(x_{W}, y_{W}\right),\left(x_{N}, y_{N}\right),\left(x_{S}, y_{S}\right),\left(x_{e}, y_{e}\right)$, $\left(x_{w}, y_{w}\right),\left(x_{n}, y_{n}\right),\left(x_{s}, y_{s}\right),\left(x_{n e}, y_{n e}\right),\left(x_{n w}, y_{n w}\right),\left(x_{s e}, y_{s e}\right)$, and $\left(x_{s w}, y_{s w}\right)$, respectively. These coordinates are not all independent of each other. Once the coordinates of interface points (e, w, $\mathrm{n}$, and s) are given, the coordinates of the other points can be determined. The coordinates of point "p," which is located at the center of the control volume, can be expressed as:

$$
\begin{aligned}
& x_{p}=\left(x_{c}+x_{w}\right) / 2 \\
& y_{p}=\left(y_{n}+y_{s}\right) / 2
\end{aligned}
$$

The coordinates of the neighboring points "E," "W," "N," and "S" and the corner points "ne," "nw," "se," and "sw" are all related to the interface and the center node points.

$$
\begin{aligned}
& x_{n e}=x_{s e}=x_{e} \\
& x_{N}=x_{n}=x_{s}=x_{s}=x_{p} \\
& x_{n w}=x_{s w}=x_{w} \\
& y_{n e}=y_{n w}=y_{n} \\
& y_{E}=y_{e}=y_{w}=y_{w}=y_{p} \\
& y_{s e}=y_{s w}=y_{s}
\end{aligned}
$$

The volume of the cell " $p$ " is defined by a length $\Delta x_{p}$, a height $\Delta y_{p}$, and a unit width. The unit width is in a direction orthogonal to both $\mathrm{x}$ and $\mathrm{y}$ axes. The cell length and height are expressed as:

$$
\begin{aligned}
& \Delta \mathrm{x}_{\mathrm{p}}=\mathrm{x}_{\mathrm{e}}-\mathrm{x}_{\mathrm{w}} \\
& \Delta \mathrm{y}_{\mathrm{p}}=\mathrm{y}_{\mathrm{n}}-\mathrm{y}_{\mathrm{s}}
\end{aligned}
$$

A grid needed for solving a governing equation does not have to be the same for all equations. The number of grids used in a CFD code depends mainly on numerical considerations. A staggered system is used for the ICOMFLO code. The staggered system has three grids: an $\mathrm{x}$-momentum grid for the momentum equation of the axial gas velocity $\mathrm{u}$, a $\mathrm{y}$ momentum grid for the momentum equation of transverse gas velocity $\mathrm{v}$, and a scalar grid for all the other equations. Note that the reference point " $p$ " of a cell in the staggered $x$ - and $y$ momentum grids may not necessarily be located at the center of a momentum cell, because the scalar cells may be defined so their rows or columns have varying heights or widths, and because the reference point " $p$ " of a momentum cell is always located on the cell interface of a scalar cell; 
when adjacent scalar cells are of different size, "p" of the overlapping momentum cell will not be in the momentum cell center in that case. A grid has interior and boundary cells. Flow properties of a boundary cell are generally given. Flow properties of an interior cell have to be determined from the governing equations. Since the solution is obtained at the reference point "p," flow properties at the interface and corner points have to be determined by interpolation (Section 4.4).

Figure 6 shows relative locations of the scalar (solid circles), x-momentum (horizontal vectors), and $\mathrm{y}$-momentum (vertical vectors) cells. The shaded areas labeled $\mathrm{S}$, $\mathrm{U}$, and $\mathrm{V}$ represent the control volumes of a scalar, an x-momentum, and a y-momentum cell, respectively. The staggered grid is used to improve the prediction of gas mass flux on the interfaces of a scalar cell. For a scalar cell, axial gas velocity $u$ is solved on the east and west interfaces "e" and "w," transverse gas velocity $v$ is solved on the north and south interfaces " $n$ " and "s," and all the other flow properties are solved at the cell center "p." The staggered grid system also yields the pressure gradient across momentum cells directly from the solution of the partial differential equation for pressure and not from interpolated values, which can be very important in maintaining numerical stability in the solution procedure.

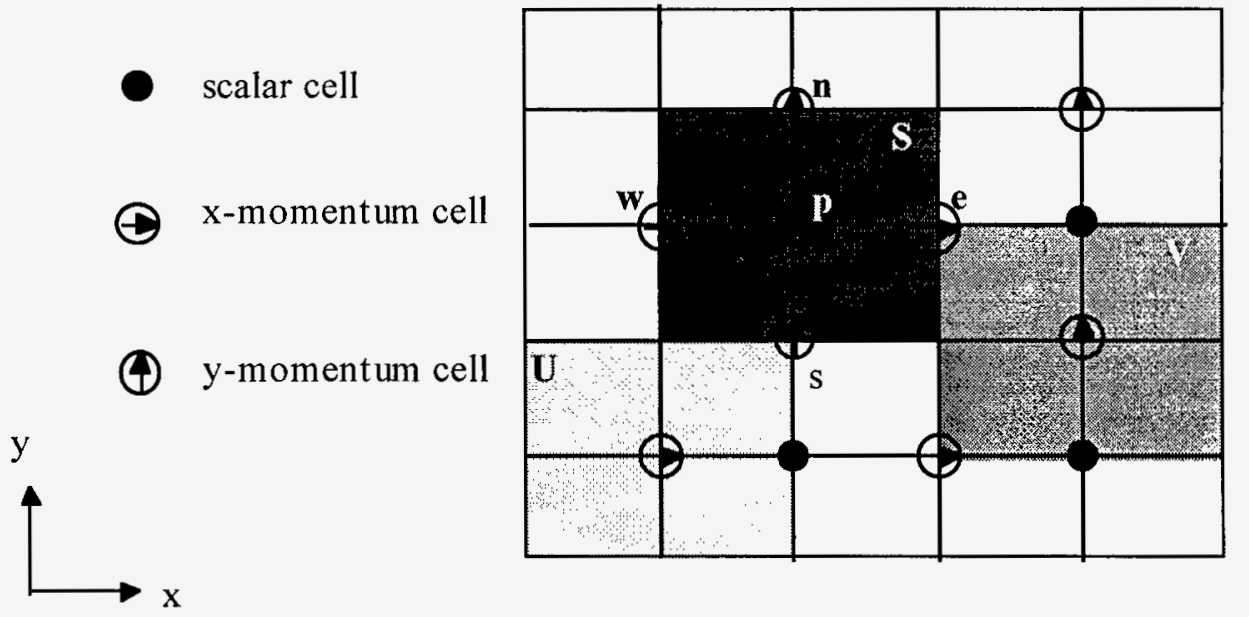

Figure 6 Relative Position of Scalar and Momentum Cells

Not all interior cells have to be included in the solution procedure; some can be defined as blocked. Since a blocked cell is impermeable to fluid flow, a computational flow domain with irregular boundary contours can be defined. Figure 7 shows a computational grid with 13 blocked cells (shaded cells), representing a flow shift upward one cell through an irregular passage. 


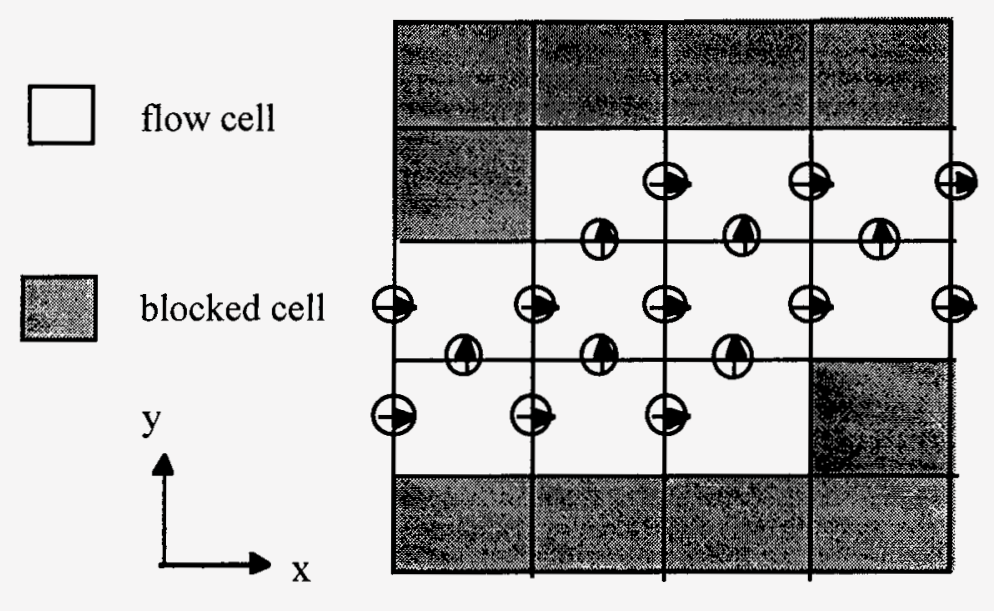

Figure 7 Blocked and Open Cells in a Grid

Flow properties of gas and condensed phases are determined from the governing equations. Some of the governing equations are partial differential equations. They need to be discretized at each computational cell and transformed into algebraic equations before solving them in an iteration scheme.

\subsection{Discretization of Gas-Phase Equations}

For the staggered grid system described in Section 4.1, gas flow properties are divided into scalar and momentum properties. The discretization processes for scalar and momentum equations are different and are discussed separately in Sections 4.2.1 and 4.2.2. Gas properties for scalar cells are solved for at the center point of a computational cell. At the other grid points, gas properties are determined by interpolation. Section 4.2.3 describe the process of interpolation.

\subsubsection{Gas Scalar Equations}

Governing equations of gas continuity, enthalpy, fuel species, richness, turbulent kinetic energy, and turbulent dissipation rate share a general formula shown as Eq. 4.5. Flow property $\xi$ of the general equation represents scalar unity number (1) for mass continuity, enthalpy (h), fuel species concentration $\left(Y_{\mathrm{f}}\right)$, richness parameter $(\phi)$, turbulent kinetic energy $(\mathrm{k})$, or turbulent dissipation rate $(\varepsilon)$. Terms containing combined convective and diffusive fluxes $\mathrm{J}_{\mathrm{x}}$ and $\mathrm{J}_{\mathrm{y}}$ are put on the left-hand side of the equation. The linearized source terms $\mathrm{S}_{\mathrm{c}}+\mathrm{S}_{\mathrm{p}} \xi$ are on the right-hand side of the equation.

$$
\frac{\partial \mathrm{J}_{\mathrm{x}}}{\partial \mathrm{x}}+\frac{\partial \mathrm{J}_{\mathrm{y}}}{\partial \mathrm{y}}=\mathrm{S}_{\mathrm{c}}+\mathrm{S}_{\mathrm{p}} \xi
$$

where the combined convective and diffusive fluxes $\mathrm{J}_{\mathrm{x}}$ and $\mathrm{J}_{\mathrm{y}}$ are defined as 


$$
\begin{aligned}
& J_{x}=f_{x} \xi-\Gamma_{\xi} \frac{\partial \xi}{\partial x} \\
& J_{y}=f_{y} \xi-\Gamma_{\xi} \frac{\partial \xi}{\partial y}
\end{aligned}
$$

For each computational cell, the differential equation can be integrated over the control volume to convert it to a discretized algebraic equation, shown as Eq. 4.6. Equation 4.6 is not linear because the coefficients $a_{p}, a_{E}, a_{W}, a_{N}, a_{S}$, and $b$ contain variables. An iteration procedure is needed to solve the equations for all scalar properties at all cells. The algebraic equation can also be expressed in a matrix form as shown in Eq. 4.7.

$$
\begin{aligned}
& a_{p} \xi_{p}=a_{E} \xi_{E}+a_{W} \xi_{W}+a_{N} \xi_{N}+a_{S} \xi_{S}+b \\
& {\left[\begin{array}{ccccc}
a_{p} & 0 & 0 & 0 & 0 \\
0 & -a_{E} & 0 & 0 & 0 \\
0 & 0 & -a_{W} & 0 & 0 \\
0 & 0 & 0 & -a_{N} & 0 \\
0 & 0 & 0 & 0 & -a_{S}
\end{array}\right]\left[\begin{array}{l}
\xi_{p} \\
\xi_{E} \\
\xi_{W} \\
\xi_{N} \\
\xi_{S}
\end{array}\right]=\left[\begin{array}{l}
b \\
0 \\
0 \\
0 \\
0
\end{array}\right]}
\end{aligned}
$$

The continuity equation states that the sum of all mass flux flowing out of a cell equals the mass generation in the cell. The convective and diffusive fluxes $J_{x}$ and $J_{y}$ of the continuity equation are simply the mass fluxes $f_{x}$ and $f_{y}$.

$$
\begin{aligned}
& \mathrm{f}_{\mathrm{x}}=\theta \rho \mathrm{u} \\
& \mathrm{f}_{\mathrm{y}}=\theta \rho \mathrm{v}
\end{aligned}
$$

Let the source term $S_{1}$ be constant in a cell of interest $p$; the continuity equation can be integrated over the control volume of the $\mathrm{p}$ cell and yields a discretized equation. Multiplying by the volume term per unit width, $\Delta \mathrm{x}_{\mathrm{p}} \Delta \mathrm{y}_{\mathrm{p}}$, the discretized equation becomes

$$
\left(f_{e}-f_{w}\right) \Delta y_{p}+\left(f_{n}-f_{s}\right) \Delta x_{p}=S_{1} \Delta x_{p} \Delta y_{p}
$$

where

$$
\begin{aligned}
& \mathrm{f}_{\mathrm{e}}=\theta_{\mathrm{e}} \rho_{\mathrm{e}} \mathrm{u}_{\mathrm{e}} \\
& \mathrm{f}_{\mathrm{w}}=\theta_{\mathrm{w}} \rho_{\mathrm{w}} \mathrm{u}_{\mathrm{w}} \\
& \mathrm{f}_{\mathrm{n}}=\theta_{\mathrm{n}} \rho_{\mathrm{n}} \mathrm{v}_{\mathrm{n}} \\
& \mathrm{f}_{\mathrm{s}}=\theta_{\mathrm{s}} \rho_{\mathrm{s}} \mathrm{v}_{\mathrm{s}}
\end{aligned}
$$


The discretized continuity equation looks simple but is the most difficult equation to be solved, because density is extremely insensitive to property changes in most flows. In most flow simulations, pressure is solved for instead of density. A pressure equation can be derived from the continuity and the momentum equations (see Section 4.4).

For other gas-phase scalar properties, a discretized equation (4.10) is similarly obtained with additional diffusive flux and linearized source terms.

$$
\left(\mathrm{J}_{\mathrm{e}}-\mathrm{J}_{\mathrm{w}}\right) \Delta \mathrm{y}_{\mathrm{p}}+\left(\mathrm{J}_{\mathrm{n}}-\mathrm{J}_{\mathrm{s}}\right) \Delta \mathrm{x}_{\mathrm{p}}=\left(\mathrm{S}_{\mathrm{c}}+\mathrm{S}_{\mathrm{p}} \xi_{\mathrm{p}}\right) \Delta \mathrm{x}_{\mathrm{p}} \Delta \mathrm{y}_{\mathrm{p}}
$$

The combined convective and diffusive fluxes $J_{e}, J_{w}, J_{n}$, and $J_{s}$ can be determined through various numerical methods (i.e., difference, upwind, hybrid, power law, and exponential schemes). For the best numerical stability and accuracy [5], the exponential scheme is used here. The power law scheme is an approximation of the exponential scheme meant to save computer time. However, since most modern computers used for engineering applications provide for computation of the exponential function in hardware, no time is saved by using the approximating power law scheme. Equation 4.11 shows the axial flux $J_{\mathrm{e}}$ on the east interface of the scalar cell "p." The convective term is a product of mass flux $f_{e}$ on the interface "e" and flow property $\xi$ of the upwind cell for this direction(east/west). The choice of the upwind cell depends on the flow direction. If the mass flows from cell $\mathrm{E}$ to cell $\mathrm{p}\left(\mathrm{f}_{\mathrm{e}}\right.$ is negative), $\xi_{\mathrm{E}}$ is used. Conversely, if the mass flows from cell $p$ to cell $E$ ( $f_{e}$ is positive), $\xi_{p}$ is used. The choice of the upwind cell is represented by using a special function "max," defined in Eq. 4.11a. A grid cell Peclet number function $\mathrm{A}\left(\mathrm{P}_{\mathrm{e}}\right)$ expressed in exponential form as shown in Eq. $4.11 \mathrm{~b}$ is used in the flux equation, Eq. $4.11 \mathrm{a}$, as a weighting function of the diffusive term. The Peclet number defined in Eq. $4.11 \mathrm{c}$ is a ratio of the convective flux to the diffusive flux between neighboring points on the grid. When $\mathrm{P}_{e}$ is large, the weighting function $A\left(\mathrm{P}_{e}\right)$ approaches zero and the convective terms dominate the flux expression. When $\mathrm{P}_{\mathrm{e}}$ approaches zero, the weighting function $\mathrm{A}\left(\mathrm{P}_{\mathrm{e}}\right)$ approaches unity and the diffusive term dominates the flux expression.

$$
\mathrm{J}_{\mathrm{e}}=\max \left(0, \mathrm{f}_{\mathrm{e}}\right) \xi_{\mathrm{p}}-\max \left(-\mathrm{f}_{\mathrm{e}}, 0\right) \xi_{\mathrm{E}}+\Gamma_{\xi} \frac{\left(\xi_{\mathrm{p}}-\xi_{E}\right)}{\Delta \mathrm{x}_{E}} \mathrm{~A}\left(\mathrm{P}_{\mathrm{e}, \mathrm{e}}\right)
$$

where:

$$
\begin{aligned}
& \max (a, b)=\text { the larger of } a \text { and } b \\
& A\left(P_{e}\right)= \begin{cases}P_{e} /\left[\exp \left(P_{e}\right)-1\right] & \text { for } P_{e}>0 \\
1-P_{e} / 2 & \text { for } P_{e} \rightarrow 0\end{cases} \\
& P_{e, e}=\frac{f_{e} \Delta x_{E}}{\Gamma_{\xi e}}
\end{aligned}
$$

Equations $4.12,4.13$, and 4.14 show the flux terms on the interfaces $w, s$, and $n$, respectively.

$$
J_{w}=\max \left(0, f_{w}\right) \xi_{w}-\max \left(-f_{w}, 0\right) \xi_{p}+\Gamma_{\xi} \frac{\left(\xi_{w}-\xi_{p}\right)}{\Delta x_{w}} A\left(P_{e, w}\right)
$$




$$
\begin{aligned}
& J_{n}=\max \left(0, f_{n}\right) \xi_{p}-\max \left(-f_{n}, 0\right) \xi_{N}+\Gamma_{\xi} \frac{\left(\xi_{p}-\xi_{N}\right)}{\Delta y_{N}} A\left(P_{e, n}\right) \\
& J_{s}=\max \left(0, f_{s}\right) \xi_{S}-\max \left(-f_{s}, 0\right) \xi_{p}+\Gamma_{\xi} \frac{\left(\xi_{s}-\xi_{p}\right)}{\Delta y_{S}} A\left(P_{e, s}\right)
\end{aligned}
$$

where Peclet numbers are defined as

$$
\begin{aligned}
& P_{e, w}=\frac{f_{w} \Delta x_{w}}{\Gamma_{\xi, w}} \\
& P_{e, n}=\frac{f_{n} \Delta x_{N}}{\Gamma_{\xi, n}} \\
& P_{e, s}=\frac{f_{s} \Delta x_{S}}{\Gamma_{\xi, s}}
\end{aligned}
$$

The discretized Eq. 4.10 can be further simplified by combining it with the continuity equation. Multiplying the discretized continuity equation, Eq. 4.9, by $\xi_{p}$ and subtracting from the flux equation, Eq. 4.10, the discretized equation becomes:

$$
\begin{aligned}
\left(J_{e}\right. & \left.-f_{e} \xi_{p}\right) \Delta y_{p}-\left(J_{w}-f_{w} \xi_{p}\right) \Delta y_{p}+\left(J_{n}-f_{n} \xi_{p}\right) \Delta x_{p}-\left(J_{s}-f_{s} \xi_{p}\right) \Delta x_{p} \\
& =\left[S_{c}+\xi_{p}\left(S_{p}-S_{1}\right)\right] \Delta x_{p} \Delta y_{p}
\end{aligned}
$$

After rearranging Eq. 4.15, a discretized algebraic equation like Eq. 4.6 is obtained.

$$
a_{p} \xi_{p}=a_{E} \xi_{E}+a_{w} \xi_{W}+a_{N} \xi_{N}+a_{s} \xi_{s}+b
$$

The coefficients of the algebraic equation are:

$$
\begin{aligned}
& \mathrm{a}_{\mathrm{E}}=\left[\max \left(0,-\mathrm{f}_{\mathrm{e}}\right)+\mathrm{A}\left(\mathrm{P}_{\mathrm{e}, \mathrm{e}}\right) \Gamma_{\xi} / \Delta \mathrm{x}_{\mathrm{E}}\right] \Delta \mathrm{y}_{\mathrm{p}} \\
& \mathrm{a}_{\mathrm{W}}=\left[\max \left(0, \mathrm{f}_{\mathrm{w}}\right)+\mathrm{A}\left(\mathrm{P}_{\mathrm{e}, \mathrm{w}}\right) \Gamma_{\xi} / \Delta \mathrm{x}_{\mathrm{W}}\right] \Delta \mathrm{y}_{\mathrm{p}} \\
& \mathrm{a}_{\mathrm{N}}=\left[\max \left(0,-\mathrm{f}_{\mathrm{n}}\right)+\mathrm{A}\left(\mathrm{P}_{\mathrm{e}, \mathrm{n}}\right) \Gamma_{\xi} / \Delta \mathrm{y}_{\mathrm{N}}\right] \Delta \mathrm{x}_{\mathrm{p}} \\
& \mathrm{a}_{\mathrm{S}}=\left[\max \left(0, \mathrm{f}_{\mathrm{s}}\right)+A\left(\mathrm{P}_{\mathrm{e}, \mathrm{S}}\right) \Gamma_{\xi} / \Delta \mathrm{y}_{\mathrm{S}}\right] \Delta \mathrm{x}_{\mathrm{p}} \\
& \mathrm{a}_{\mathrm{p}}=\mathrm{a}_{\mathrm{E}}+\mathrm{a}_{\mathrm{w}}+\mathrm{a}_{\mathrm{N}}+\mathrm{a}_{\mathrm{S}}+\left(\mathrm{S}_{1}-\mathrm{S}_{\mathrm{p}}\right) \Delta \mathrm{x}_{\mathrm{p}} \Delta \mathrm{y}_{\mathrm{p}} \\
& \mathrm{b}=\mathrm{S}_{\mathrm{c}} \Delta \mathrm{x}_{\mathrm{p}} \Delta \mathrm{y}_{\mathrm{p}}
\end{aligned}
$$




\subsubsection{Gas Momentum Equations}

There are two gas-phase momentum equations: $x$-momentum and $y$-momentum. The $x$ momentum equation is used to solve for the axial velocity $u$ and the $y$-momentum equation for the transverse velocity $\mathrm{v}$.

Equation 4.17 shows the $x$-momentum equation. The equation is similar to the scalar equation, Eq. 4.5, except for an additional pressure gradient source term on the right-hand side of the equation.

$$
\frac{\partial J_{x}}{\partial x}+\frac{\partial J_{y}}{\partial y}=-\theta \frac{\partial p}{\partial x}+S_{c}+S_{p} u
$$

A discretized $x$-momentum equation is derived by using the integral volume approach described in previous section. The discretized $\mathrm{x}$-momentum equation has the same form as Eq. 4.6, in which the flow property is $\mathrm{u}$.

$$
a_{p} u_{p}=a_{E} u_{E}+a_{W} u_{W}+a_{N} u_{N}+a_{S} u_{S}+b
$$

The coefficients of the discretized $x$-momentum equation are:

$$
\begin{aligned}
& a_{E}=\left[\max \left(0,-f_{e}\right)+A\left(P_{e, e}\right) \Gamma_{\xi} / \Delta x_{E}\right] \Delta y_{p} \\
& \mathrm{a}_{\mathrm{w}}=\left[\max \left(0, \mathrm{f}_{\mathrm{w}}\right)+\mathrm{A}\left(\mathrm{P}_{\mathrm{e}, \mathrm{w}}\right) \Gamma_{\xi} / \Delta \mathrm{x}_{\mathrm{w}}\right] \Delta \mathrm{y}_{\mathrm{p}} \\
& a_{N}=\left[\max \left(0,-f_{n w}\right)+A\left(P_{e, n w}\right) \Gamma_{\xi} / \Delta y_{N}\right]\left(x_{p}-x_{w}\right) \\
& +\left[\max \left(0,-\mathrm{f}_{\mathrm{ne}}\right)+\mathrm{A}\left(\mathrm{P}_{\mathrm{e}, \mathrm{ne}}\right) \Gamma_{\xi} / \Delta \mathrm{y}_{\mathrm{N}}\right]\left(\mathrm{x}_{\mathrm{e}}-\mathrm{x}_{\mathrm{p}}\right) \\
& a_{\mathrm{s}}=\left[\max \left(0, \mathrm{f}_{\mathrm{sw}}\right)+\mathrm{A}\left(\mathrm{P}_{\mathrm{e}, \mathrm{sw}}\right) \Gamma_{\xi} / \Delta \mathrm{y}_{\mathrm{s}}\right]\left(\mathrm{x}_{\mathrm{p}}-\mathrm{x}_{\mathrm{w}}\right) \\
& +\left[\max \left(0, \mathrm{f}_{\mathrm{se}}\right)+\mathrm{A}\left(\mathrm{P}_{\mathrm{e}, \mathrm{se}}\right) \Gamma_{\xi} / \Delta \mathrm{y}_{\mathrm{S}}\right]\left(\mathrm{x}_{\mathrm{e}}-\mathrm{x}_{\mathrm{p}}\right) \\
& a_{p}=a_{E}+a_{W}+a_{N}+a_{S}+\left(S_{1}-S_{p}\right) \Delta x_{p} \Delta y_{p} \\
& \mathrm{~b}=\mathrm{S}_{\mathrm{c}} \Delta \mathrm{x}_{\mathrm{p}} \Delta \mathrm{y}_{\mathrm{p}}+\theta_{\mathrm{p}}\left(\mathrm{p}_{\mathrm{w}}-\mathrm{p}_{\mathrm{e}}\right) \Delta \mathrm{y}_{\mathrm{p}}
\end{aligned}
$$

Figure 8 shows the labeling of the neighboring points in an $\mathrm{x}$-momentum cell. Note that an $\mathrm{x}$-momentum cell differs from a scalar cell. The mass flux on the north interface of an $\mathrm{x}$ momentum cell consists of a mass flux from the northwest corner and the other mass flux from the northeast corner, because scalar properties are defined on the east and west interfaces. Equation $4.18 \mathrm{c}$ shows that coefficient $\mathrm{a}_{\mathrm{N}}$ includes both fluxes from nw and ne corners. Similarly, Eq. $4.18 \mathrm{~d}$ shows that coefficient as includes both fluxes from sw and se corners. 
scalar cell

e x-momentum cell
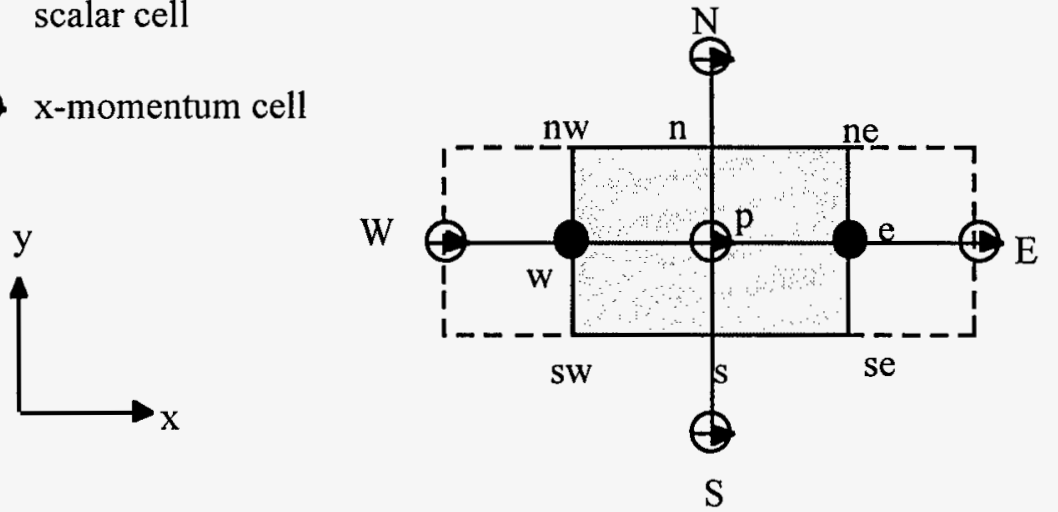

Figure 8 Labeling of an X-momentum Cell

If an $\mathrm{x}$-momentum cell is next to a boundary or a blocked cell, the $\mathrm{x}$-momentum cell needs to be extended an extra half cell to cover the entire flow field. Figure 9 shows an extended $x-$ momentum cell, which consists of one and one-half scalar cells.

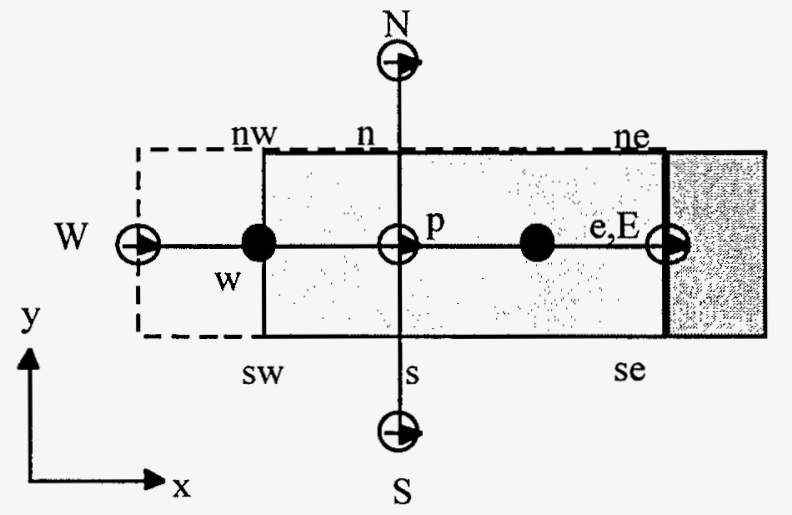

scalar point

$\Theta x$-momentum point

boundary or blocked cell

Figure 9 An Extended X-momentum Cell

Equation 4.19 shows the $y$-momentum equation. Again, the equation is similar to the scalar equation, Eq. 4.5, except for an additional pressure gradient source term in the right-hand side of the equation.

$$
\frac{\partial \mathrm{J}_{\mathrm{x}}}{\partial \mathrm{x}}+\frac{\partial \mathrm{J}_{\mathrm{y}}}{\partial \mathrm{y}}=-\theta \frac{\partial \mathrm{p}}{\partial \mathrm{y}}+\mathrm{S}_{\mathrm{c}}+\mathrm{S}_{\mathrm{p}} \mathrm{v}
$$

Equation 4.20 shows the discretized $y$-momentum equation derived from the integral volume approach. The discretized equation has the same form as Eq. 4.6, where the flow property is $\mathrm{v}$.

$$
a_{p} v_{p}=a_{E} v_{E}+a_{W} v_{W}+a_{N} v_{N}+a_{S} v_{S}+b
$$

The coefficients of the discretized y-momentum equation are: 


$$
\begin{aligned}
& a_{E}=\left[\max \left(0,-f_{n e}\right)+A\left(P_{e, n e}\right) \Gamma_{\xi} / \Delta x_{E}\right]\left(y_{n}-y_{p}\right) \\
& +\left[\max \left(0,-f_{s e}\right)+A\left(P_{e, s e}\right) \Gamma_{\xi} / \Delta x_{E}\right]\left(y_{p}-y_{s}\right) \\
& \mathrm{a}_{\mathrm{W}}=\left[\max \left(0, \mathrm{f}_{\mathrm{nw}}\right)+A\left(\mathrm{P}_{\mathrm{e}, \mathrm{nw}}\right) \Gamma_{\xi} / \Delta \mathrm{x}_{\mathrm{W}}\right]\left(\mathrm{y}_{\mathrm{n}}-\mathrm{y}_{\mathrm{p}}\right) \\
& +\left[\max \left(0, \mathrm{f}_{\mathrm{sw}}\right)+A\left(\mathrm{P}_{\mathrm{e}, \mathrm{sw}}\right) \Gamma_{\xi} / \Delta \mathrm{x}_{\mathrm{W}}\right]\left(\mathrm{y}_{\mathrm{p}}-\mathrm{y}_{\mathrm{s}}\right) \\
& a_{N}=\left[\max \left(0,-f_{n}\right)+A\left(P_{e, n}\right) \Gamma_{\xi} / \Delta y_{N}\right] \Delta x_{p} \\
& \mathrm{a}_{\mathrm{S}}=\left[\max \left(0, \mathrm{f}_{\mathrm{s}}\right)+A\left(\mathrm{P}_{\mathrm{e}, \mathrm{S}}\right) \Gamma_{\xi} / \Delta \mathrm{y}_{\mathrm{S}}\right] \Delta \mathrm{x}_{\mathrm{p}} \\
& a_{p}=a_{E}+a_{w}+a_{N}+a_{S}+\left(S_{1}-S_{p}\right) \Delta x_{p} \Delta y_{p} \\
& b=S_{c} \Delta x_{p} \Delta x_{p}+\theta_{p}\left(p_{n}-p_{s}\right) \Delta x_{p}
\end{aligned}
$$

Figure 10 shows the labeling of the neighboring points in a y-momentum cell. Note that a $y$-momentum cell differs from a scalar cell. The mass flux on the east interface of a $y-$ momentum cell consists of a mass flux from the northeast corner and the other mass flux from the southeast corner, because scalar properties are defined on the north and south interfaces. Equation 4.20a shows that coefficient $a_{N}$ includes both fluxes from ne and se corners. Similarly, Eq. $4.20 \mathrm{~b}$ shows that coefficient $\mathrm{a}_{\mathrm{W}}$ includes both fluxes from $\mathrm{nw}$ and sw corners.

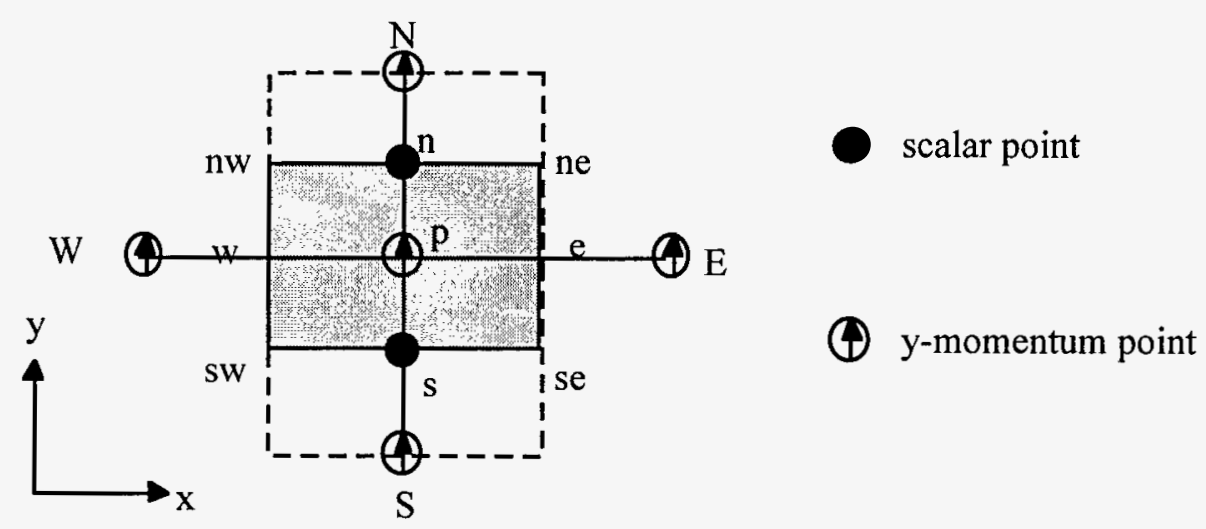

Figure 10 Labeling of a Y-momentum Cell 


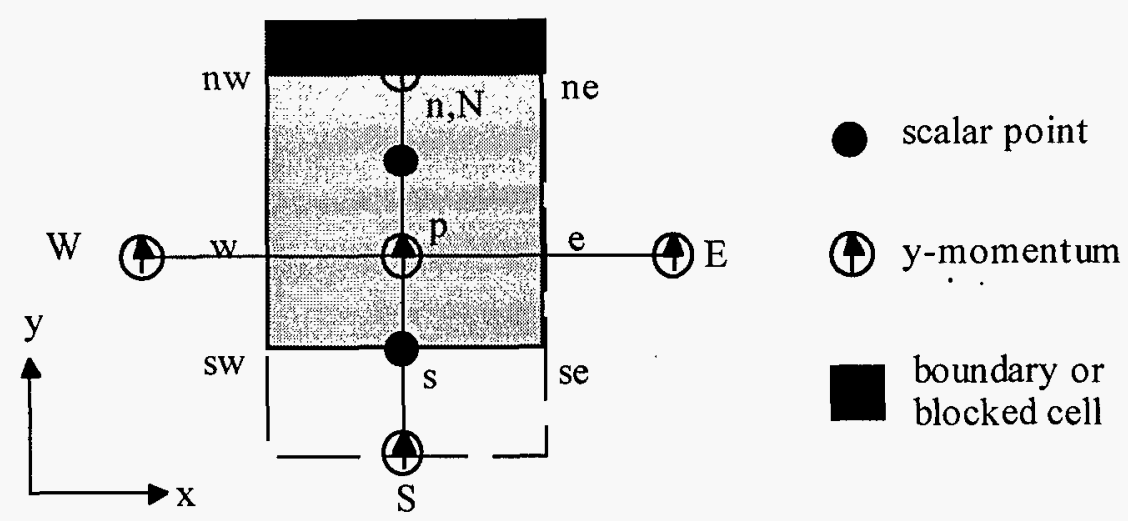

Figure 11 An Extended Y-momentum Cell

If a y-momentum cell is next to a boundary or a blocked cell, the y-momentum cell needs to be extended an extra half cell to cover the entire flow field. Figure 11 shows an extended ymomentum cell. The control volume of an extended y-momentum cell includes one and one-half scalar cells.

\subsubsection{Interpolation of Flow Properties}

Flow properties are determined for all grid points directly by solving the governing equations in a staggered multigrid system. Those at a cell center (p, E, W, N, or S) are solved, but those on the interfaces (e, w, n, and s) or corners (nw, ne, sw, and se) are interpolated from the neighboring cells. The robustness of the computational method is in part determined by the choice of interpolation functions. To conserve computer resources and reduce program complexity, the simplest interpolation functions that provide physically reasonable results are sought. Two types of interpolations are used here for a flow property $\xi=\mathrm{p}, \mathrm{u}, \mathrm{v}, \mathrm{h}, \mathrm{Y}_{\mathrm{f}}, \phi, \mathrm{k}, \varepsilon$, $n_{i}, u_{i}, v_{i}$, or $T_{i}$ and a diffusion coefficient $\Gamma$.

For a flow property $\xi$, the interface value is determined by linear interpolation. The property value on the east interface, for example, can be determined from the neighboring cells $p$ and $\mathrm{E}$ as shown in Eq. 4.21a. Similarly, interpolation values on the interfaces $\mathrm{w}, \mathrm{s}$, and $\mathrm{n}$ are derived as Eqs. $4.21 \mathrm{~b}, 4.21 \mathrm{c}$, and $4.21 \mathrm{~d}$, respectively.

$$
\begin{aligned}
& \xi_{e}=\left(\frac{x_{e}-x_{E}}{x_{p}-x_{E}}\right) \xi_{P}+\left(\frac{x_{p}-x_{e}}{x_{p}-x_{E}}\right) \xi_{E} \\
& \xi_{w}=\left(\frac{x_{W}-x_{w}}{x_{W}-x_{p}}\right) \xi_{p}+\left(\frac{x_{w}-x_{p}}{x_{W}-x_{p}}\right) \xi_{W} \\
& \xi_{s}=\left(\frac{x_{s}-x_{S}}{x_{p}-x_{S}}\right) \xi_{P}+\left(\frac{x_{p}-x_{s}}{x_{p}-x_{S}}\right) \xi_{S}
\end{aligned}
$$




$$
\xi_{n}=\left(\frac{x_{N}-x_{n}}{x_{N}-x_{p}}\right) \xi_{P}+\left(\frac{x_{n}-x_{p}}{x_{N}-x_{p}}\right) \xi_{N}
$$

For a diffusion coefficient $\Gamma$, the value on the cell interface is determined by the harmonic mean. Equation 4.22a shows the interpolation formula for the interface e. In this formulation, if either $\Gamma_{\mathrm{p}}$ or $\Gamma_{\mathrm{E}}$ approaches zero, $\Gamma_{\mathrm{e}}$ also approaches zero, and if one of $\Gamma_{\mathrm{p}}$ or $\Gamma_{\mathrm{E}}$ is orders of magnitude larger than the other, $\Gamma_{\mathrm{e}}$ becomes very close to the smaller value of the two. Therefore, the harmonic mean provides the correct physical result in the limiting cases for diffusion, while linear interpolation yields incorrect physical results in the limiting cases, and for this reason calculation of the harmonic mean is the chosen method for interpolating diffusion coefficients. Equations $4.22 \mathrm{~b}, 4.22 \mathrm{c}$, and $4.22 \mathrm{~d}$ show the interpolation formula for the interfaces $\mathrm{w}, \mathrm{s}$, and $\mathrm{n}$, respectively.

$$
\begin{aligned}
& \frac{1}{\Gamma_{e}}=\left(\frac{x_{e}-x_{E}}{x_{p}-x_{E}}\right) \frac{1}{\Gamma_{p}}+\left(\frac{x_{p}-x_{e}}{x_{p}-x_{E}}\right) \frac{1}{\Gamma_{E}} \\
& \frac{1}{\Gamma_{w}}=\left(\frac{x_{W}-x_{w}}{x_{W}-x_{p}}\right) \frac{1}{\Gamma_{p}}+\left(\frac{x_{w}-x_{p}}{x_{W}-x_{p}}\right) \frac{1}{\Gamma_{W}} \\
& \frac{1}{\Gamma_{s}}=\left(\frac{x_{s}-x_{S}}{x_{p}-x_{S}}\right) \frac{1}{\Gamma_{p}}+\left(\frac{x_{p}-x_{s}}{x_{p}-x_{S}}\right) \frac{1}{\Gamma_{S}} \\
& \frac{1}{\Gamma_{n}}=\left(\frac{x_{N}-x_{n}}{x_{N}-x_{p}}\right) \frac{1}{\Gamma_{p}}+\left(\frac{x_{n}-x_{p}}{x_{N}-x_{p}}\right) \frac{1}{\Gamma_{N}}
\end{aligned}
$$

\subsection{Discretization of Condensed-Phase Equations}

Governing equations for the condensed phase include those of particle number density, $x$ momentum, $y$-momentum, and energy. The particle number density equation is different than the others. Discretization of the particle number density equation and the other equations is presented below.

\subsubsection{Particle Number Density Equation}

The number density equation of the $i^{\text {th }}$ particle lump is

$$
\frac{\partial J_{i, x}}{\partial x}+\frac{\partial J_{i, y}}{\partial y}=S_{c}+S_{p} n_{i}
$$

The flux terms $\mathrm{J}_{\mathrm{x}, \mathrm{i}}$ and $\mathrm{J}_{\mathrm{y}, \mathrm{i}}$ are defined as follows:

$$
\mathrm{J}_{\mathrm{i}, \mathrm{x}}=\mathrm{n}_{\mathrm{i}} \mathrm{u}_{\mathrm{i}}-\Gamma_{\mathrm{n}, \mathrm{i}} \frac{\partial \mathrm{n}_{\mathrm{i}}}{\partial \mathrm{x}}
$$




$$
\mathrm{J}_{\mathrm{i}, \mathrm{y}}=\mathrm{n}_{\mathrm{i}} \mathrm{v}_{\mathrm{i}}-\Gamma_{\mathrm{n}, \mathrm{i}} \frac{\partial \mathrm{n}_{\mathrm{i}}}{\partial \mathrm{y}}
$$

where the turbulent diffusivity $\Gamma_{\mathrm{n}, \mathrm{i}}$ is defined in Eq. (3.18).

Scalar cells (see Fig. 5) are used to solve all of the condensed-phase governing equations because there is no pressure gradient term in the condensed-phase momentum equations. In using the control volume approach, Eq. 4.23 is integrated over the control volume of a computational cell $\mathrm{p}$, yielding a discretized equation. Multiplying the discretized equation with the volume $\Delta x_{p} \Delta y_{p}$, it becomes

$$
\left(J_{i, e}-J_{i, w}\right) \Delta y_{p}+\left(J_{i, n}-J_{i, s}\right) \Delta x_{p}=\left(S_{c}+S_{p} n_{i, p}\right) \Delta x_{p} \Delta y_{p}
$$

where the fluxes are defined as

$$
\begin{aligned}
& J_{i, e}=\max \left(0, f_{i, e}\right) n_{i, p}-\max \left(-f_{i, e}, 0\right) n_{i, E}+\Gamma_{n, i} \frac{\left(n_{i, p}-n_{i, E}\right)}{\Delta x_{E}} A\left(P_{e, e}\right) \\
& J_{i, w}=\max \left(0, f_{i, w}\right) n_{i, w}-\max \left(-f_{i, w}, 0\right) n_{i, p}+\Gamma_{n, i} \frac{\left(n_{i, w}-n_{i, p}\right)}{\Delta x_{W}} A\left(P_{e, w}\right) \\
& J_{i, n}=\max \left(0, f_{i, n}\right) n_{i, p}-\max \left(-f_{i, n}, 0\right) n_{i, N}+\Gamma_{n, i} \frac{\left(n_{i, p}-n_{i, N}\right)}{\Delta y_{N}} A\left(P_{e, n}\right) \\
& J_{i, s}=\max \left(0, f_{i, s}\right) n_{i, S}-\max \left(-f_{i, s}, 0\right) n_{i, p}+\Gamma_{n, i} \frac{\left(n_{i, S}-n_{i, p}\right)}{\Delta y_{S}} A\left(P_{e, s}\right)
\end{aligned}
$$

The mass fluxes of the condensed phase equation are defined as

$$
\begin{aligned}
& f_{i, e}=u_{i, e} \\
& f_{i, w}=u_{i, w} \\
& f_{i, n}=v_{i, n} \\
& f_{i, s}=v_{i, s}
\end{aligned}
$$

Equation 4.26 shows the discretized algebraic form of the particle number density equation. The discretized number density equation has the same form as Eq. 4.6, in which the flow property is $\mathrm{n}_{\mathrm{i}}$.

$$
a_{p} n_{i, p}=a_{E} n_{i, E}+a_{W} n_{i, W}+a_{N} n_{i, N}+a_{S} n_{i, S}+b
$$

The coefficients of the equation are:

$$
a_{E}=\max \left(0,-f_{i, e}\right)+A\left(P_{e, e}\right) \Gamma_{n, i} / \Delta x_{E}
$$




$$
\begin{aligned}
& a_{W}=\max \left(0, f_{i, w}\right)+A\left(P_{e, w}\right) \Gamma_{\xi} / \Delta x_{W} \\
& a_{N}=\max \left(0,-f_{i, n}\right)+A\left(P_{e, n}\right) \Gamma_{\xi} / \Delta y_{N} \\
& a_{S}=\max \left(0, f_{i, s}\right)+A\left(P_{e, s}\right) \Gamma_{\xi} / \Delta y_{S} \\
& a_{p}=a_{E}+a_{W}+a_{N}+a_{S}+\left(f_{i, e}-f_{i, w}+f_{i, n}-f_{i, s}\right)-S_{p} \Delta x_{p} \Delta y_{p} \\
& b=S_{c} \Delta x_{p} \Delta y_{p}
\end{aligned}
$$

\subsubsection{Particle Momentum and Energy Equations}

For particle velocity components $u_{i}$ and $v_{i}$ and temperature $T_{i}$ of the $i^{\text {th }}$ particle lump, the governing equation are written as

$$
\frac{\partial \mathrm{J}_{i, \mathrm{x}}}{\partial \mathrm{x}}+\frac{\partial \mathrm{J}_{\mathrm{i}, \mathrm{y}}}{\partial \mathrm{y}}-\xi\left(\frac{\partial \mathrm{f}_{\mathrm{i}, \mathrm{x}}}{\partial \mathrm{x}}+\frac{\partial \mathrm{f}_{\mathrm{i}, \mathrm{y}}}{\partial \mathrm{y}}\right)=\mathrm{S}_{\mathrm{c}}+\mathrm{S}_{\mathrm{p}} \xi
$$

Using the control volume integration approach, the particle property equation becomes

$$
\begin{aligned}
& \left(J_{i, e}-J_{i, w}\right) \Delta y_{p}+\left(J_{i, n}-J_{i, s}\right) \Delta x_{p}-\xi_{p}\left(f_{i, e}-f_{i, w}\right) \Delta y_{p}-\xi_{p}\left(J_{i, n}-J_{i, s}\right) \Delta x_{p} \\
& =\left(S_{c}+S_{p} n_{i, p}\right) \Delta x_{p} \Delta y_{p}
\end{aligned}
$$

Note that the diffusivity coefficient in the property flux term $\mathrm{J}$ is set to zero in the particle momentum and energy equations. Because particle momentum and energy are carried by the particles themselves and cannot be transported independently of the particles, gradients of these particle properties cannot lead to turbulent dispersion of the property independent of turbulent dispersion of the particles themselves. Equation 4.29 shows the discretized algebraic form of the particle property equation. The discretized equation has the same form as Eq. 4.6, in which the flow property is $\mathrm{u}_{\mathrm{i}}, \mathrm{v}_{\mathrm{i}}$, or $\mathrm{T}_{\mathrm{i}}$.

$$
a_{p} \xi_{p}=a_{E} \xi_{E}+a_{W} \xi_{W}+a_{N} \xi_{N}+a_{S} \xi_{S}+b
$$

The coefficients of the equation are

$$
\begin{aligned}
& a_{E}=\max \left(0,-f_{i, e}\right) \\
& a_{W}=\max \left(0, f_{i, w}\right) \\
& a_{N}=\max \left(0,-f_{i, n}\right) \\
& a_{S}=\max \left(0, f_{i, s}\right) \\
& a_{p}=a_{E}+a_{W}+a_{N}+a_{S}-S_{p} \Delta x_{p} \Delta y_{p}
\end{aligned}
$$


$\mathrm{b}=\mathrm{S}_{\mathrm{c}} \Delta \mathrm{x}_{\mathrm{p}} \Delta \mathrm{y}_{\mathrm{p}}$

\subsection{Iterative Solution Routine}

The partial differential governing equations are discretized into algebraic equations in Sections 4.2 and 4.3. Because the discretized equations are coupled, nonlinear equations and because of the large number of equations, the only practical way to solve them is to employ numerical methods to solve them iteratively on a computer. A successful iterative scheme proceeds from an initial guess of unknowns through a set of intermediate, tentative values to a converged solution. In the process used here, one equation is solved for one flow property at a time. For gas-phase flow properties, the continuity equation is used to solve for pressure, the momentum equations for velocity components, the enthalpy equation for enthalpy, the species equations for species concentrations, and the turbulence equations for turbulence variables. The state equations are used to calculate gas density and temperature. For condensed-phase flow properties, the number density equations are used to solve for particle number densities, the momentum equations for velocity components, and the energy equation for particle temperature.

\subsubsection{Pressure Equation}

Most flows are nearly incompressible, so the pressure is highly sensitive to mass imbalances. Therefore, the pressure needs to be calculated carefully from a conservation equation, which is the continuity equation. After solving for the pressure, the density can be easily calculated from the ideal gas state equation. The continuity equation cannot be used directly to determine pressure, because it does not contain pressure explicitly as a variable. However, the continuity equation does determine pressure indirectly, because a velocity field obtained by solution of the momentum equations with an incorrect pressure field will not satisfy the continuity equation.

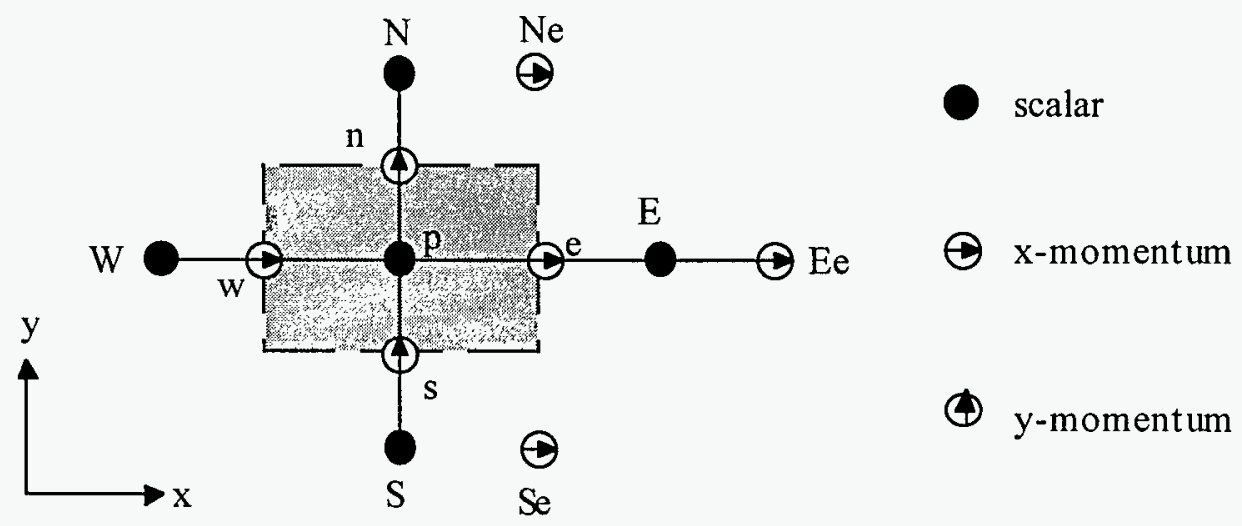

Figure 12 Labeling of a Computational Cell for Pressure Equation 
A SIMPLER algorithm developed by Patankar [27] is used here to solve for the pressure. Figure 12 shows the labeling of a computational cell for solving for pressure. The discretized $\mathrm{x}$-momentum equation, Eq. 4.20, was derived for a reference point "p" in Fig. 8 corresponding to the point "e" in Fig. 12. It can be rewritten for the point "e" in Fig. 12.

$$
a_{e} u_{e}=a_{E e} u_{E e}+a_{w} u_{w}+a_{N e} u_{N e}+a_{S e} u_{S e}+b_{e}+\theta_{e}\left(p_{p}-p_{E}\right) \Delta y_{p}
$$

The form of Eq. 4.30 can be simplified as

$$
\mathrm{u}_{\mathrm{e}}=\widetilde{\mathrm{u}}_{\mathrm{e}}+\theta_{\mathrm{e}}\left(\mathrm{p}_{\mathrm{p}}-\mathrm{p}_{\mathrm{E}}\right) \Delta \mathrm{y}_{\mathrm{p}} / \mathrm{a}_{\mathrm{e}}
$$

where a pseudovelocity $\widetilde{\mathrm{u}}_{\mathrm{e}}$ is introduced:

$$
\widetilde{u}_{e}=\left(a_{E e} u_{E e}+a_{w} u_{w}+a_{N e} u_{N e}+a_{S e} u_{S e}+b_{e}\right) / a_{e}
$$

Thus, the axial velocity on the interface "e" is expressed as Eq. 4.31. Similarly, the axial velocity on the interface "w," and transverse velocities on the interfaces " $n$ " and "s," are derived as

$$
\begin{aligned}
& \mathrm{u}_{\mathrm{w}}=\widetilde{\mathrm{u}}_{\mathrm{w}}+\theta_{\mathrm{w}}\left(\mathrm{p}_{\mathrm{w}}-\mathrm{p}_{\mathrm{p}}\right) \Delta \mathrm{y}_{\mathrm{p}} / \mathrm{a}_{\mathrm{w}} \\
& \mathrm{v}_{\mathrm{n}}=\widetilde{\mathrm{v}}_{\mathrm{n}}+\theta_{\mathrm{n}}\left(\mathrm{p}_{\mathrm{p}}-\mathrm{p}_{\mathrm{N}}\right) \Delta \mathrm{x}_{\mathrm{p}} / \mathrm{a}_{\mathrm{n}} \\
& \mathrm{v}_{\mathrm{s}}=\widetilde{\mathrm{v}}_{\mathrm{s}}+\theta_{\mathrm{s}}\left(\mathrm{p}_{\mathrm{s}}-\mathrm{p}_{\mathrm{p}}\right) \Delta \mathrm{x}_{\mathrm{p}} / \mathrm{a}_{\mathrm{s}}
\end{aligned}
$$

Note that $\widetilde{\mathrm{u}}_{\mathrm{w}}, \widetilde{\mathrm{v}}_{\mathrm{n}}$, and $\widetilde{\mathrm{v}}_{\mathrm{s}}$ are pseudovelocities defined in a similar manner to that of Eq. 4.31a.

Once the interface velocities $u_{e}, u_{w}, v_{n}$, and $v_{s}$ are determined, the interface mass fluxes $f_{e}$, $f_{w}, f_{n}$, and $f_{s}$ can be obtained.

$$
\begin{aligned}
& f_{e}=\rho_{e} \theta_{e}\left[\widetilde{u}_{e}+\theta_{e}\left(p_{p}-p_{E}\right) \Delta y_{p} / a_{e}\right] \\
& f_{w}=\rho_{w} \theta_{w}\left[\widetilde{u}_{w}+\theta_{w}\left(p_{w}-p_{p}\right) \Delta y_{p} / a_{w}\right] \\
& f_{n}=\rho_{n} \theta_{n}\left[\widetilde{v}_{n}+\theta_{n}\left(p_{p}-p_{N}\right) \Delta x_{p} / a_{n}\right] \\
& f_{s}=\rho_{s} \theta_{s}\left[\widetilde{v}_{s}+\theta_{s}\left(p_{s}-p_{p}\right) \Delta x_{p} / a_{s}\right]
\end{aligned}
$$

Now, substituting Eqs. 4.35a-4.35d into the continuity equation, Eq. 4.9, a pressure equation is obtained. After rearrangement, the pressure equation can be written in linearized form as

$$
a_{p} p_{p}=a_{E} p_{E}+a_{W} p_{W}+a_{N} p_{N}+a_{S} p_{S}+b
$$

where

$$
a_{E}=\theta_{e}^{2} \rho_{e} \Delta y_{p}^{2} / a_{e}
$$




$$
\begin{aligned}
& a_{\mathrm{w}}=\theta_{\mathrm{w}}^{2} \rho_{\mathrm{w}} \Delta \mathrm{y}_{\mathrm{p}}^{2} / \mathrm{a}_{\mathrm{w}} \\
& \mathrm{a}_{\mathrm{N}}=\theta_{\mathrm{n}}^{2} \rho_{\mathrm{n}} \Delta \mathrm{x}_{\mathrm{p}}^{2} / \mathrm{a}_{\mathrm{n}} \\
& \mathrm{a}_{\mathrm{S}}=\theta_{\mathrm{s}}^{2} \rho_{\mathrm{s}} \Delta \mathrm{x}_{\mathrm{p}}^{2} / \mathrm{a}_{\mathrm{s}} \\
& \mathrm{a}_{\mathrm{p}}=\mathrm{a}_{\mathrm{E}}+\mathrm{a}_{\mathrm{w}}+\mathrm{a}_{\mathrm{N}}+\mathrm{a}_{\mathrm{S}} \\
& \mathrm{b}=\left(\theta_{\mathrm{e}} \rho_{\mathrm{e}} \widetilde{\mathrm{u}}_{\mathrm{e}}-\theta_{\mathrm{w}} \rho_{\mathrm{w}} \widetilde{\mathrm{u}}_{\mathrm{w}}\right) \Delta \mathrm{y}_{\mathrm{p}}+\left(\theta_{\mathrm{n}} \rho_{\mathrm{n}} \widetilde{\mathrm{v}}_{\mathrm{n}}-\theta_{\mathrm{s}} \rho_{\mathrm{s}} \widetilde{\mathrm{v}}_{\mathrm{s}}\right) \Delta \mathrm{x}_{\mathrm{p}}-\mathrm{S}_{\mathrm{l}} \Delta \mathrm{x}_{\mathrm{p}} \Delta \mathrm{y}_{\mathrm{p}}
\end{aligned}
$$

\subsubsection{Pressure Correction Equation}

Since the pressure equation, Eq. 4.36, is highly nonlinear, a pressure correction equation, Eq. 4.37, is added to the solution routine to improve numerical stability during iteration and (it is hoped) speed up convergence. The pressure correction $\mathrm{p}^{\prime}$ represents the pressure change due to the imbalance of the mass flux in a cell. The term $b^{\prime}$ is the mass residual of the gas-phase continuity equation resulting from solving the momentum equation for velocity with an imperfect pressure field. The other coefficients of the pressure correction equation, $a_{E}, a_{W}, a_{N}$, $a_{S}$, and $a_{p}$, are the same as those of the pressure equation.

$$
\begin{aligned}
& a_{p} p_{p}^{\prime}=a_{E} p_{E}^{\prime}+a_{W} p^{\prime}{ }_{w}+a_{N} p_{N}^{\prime}+a_{S} p^{\prime}{ }_{s}+b^{\prime} \\
& b^{\prime}=\left(\theta_{e} \rho_{c} u_{c}-\theta_{w} \rho_{w} u_{w}\right) \Delta y_{p}+\left(\theta_{n} \rho_{n} v_{n}-\theta_{s} \rho_{s} v_{s}\right) \Delta x_{p}-S_{1} \Delta x_{p} \Delta y_{p}
\end{aligned}
$$

Since a velocity field is solved for directly from the momentum equations alone, the velocities obtained from the solution are not constrained to satisfy the continuity equation, and in general they will not satisfy it before a converged solution is obtained. However, the mass imbalance from a newly computed velocity field in an iteration can cause a great change in the pressure field and lead to a divergent computation. The pressure correction step is used to remedy this possible numerical divergence problem. Assume the new velocity field computed from the momentum equations is $\left(u^{\prime}, v^{\prime}\right)$. The velocity field can be improved by adding the pressure correction terms as shown in Eqs. 4.38a and 4.38b. Note that the pressure correction becomes zero when the velocity field satisfies the continuity equation. Note also that the solution of the pressure correction is used only to correct the velocity field, for reasons described above, and not the pressure field in the SIMPLER algorithm; rather, a new pressure field is obtained directly by solving the pressure equation using the corrected velocities in the next iteration.

$$
\begin{aligned}
& u_{p}=u_{p}^{\prime}+\theta_{p}\left(p_{w}^{\prime}-p_{e}^{\prime}\right) \Delta y_{p} / a_{p u} \\
& v_{p}=v_{p}^{\prime}+\theta_{p}\left(p_{s}^{\prime}-p_{n}^{\prime}\right) \Delta x_{p} / a_{p v}
\end{aligned}
$$




\subsubsection{Modified SIMPLER Algorithm}

A SIMPLER algorithm for solving gas-phase flow properties is constructed by Patankar [27]. The algorithm was expanded for multiphase flow calculations by Zhou and Chiu [4] and was modified extensively by Chang and Lottes $[10,13-14]$ to improve numerical stability and provide for modeling of complex multiphase reacting flows in arbitrary geometries with jet entry ports. Figure 13 shows a very general flowchart of the iterative solution routine. The basic steps of the solution algorithm are summarized as follows:

1. Calculate pseudovelocity from the discretized pseudo-momentum equation, Eq. 4.31a.

2. Solve the pressure equation, Eq. 4.36.

3. Use the pressure field obtained in step 2 to solve the discretized momentum equations, Eqs.Ê4.18 and 4.2,0 and obtain an improved velocity field.

4. Use the new velocity field from step 3 to solve the pressure correction equation, Eq. 4.38.

5. Use the pressure corrections in Eqs. 4.39a and $4.39 \mathrm{~b}$ to correct the velocity field.

6. Perform a global mass balance to maintain the free boundary conditions at the exit.

7. Solve discretized equations for other gas-phase flow properties.

8. If multiphase, solve discretized equations for condensed-phase flow properties.

9. If all equation solutions (both gas and condensed phases) have converged, exit; if not, return to step 1 .

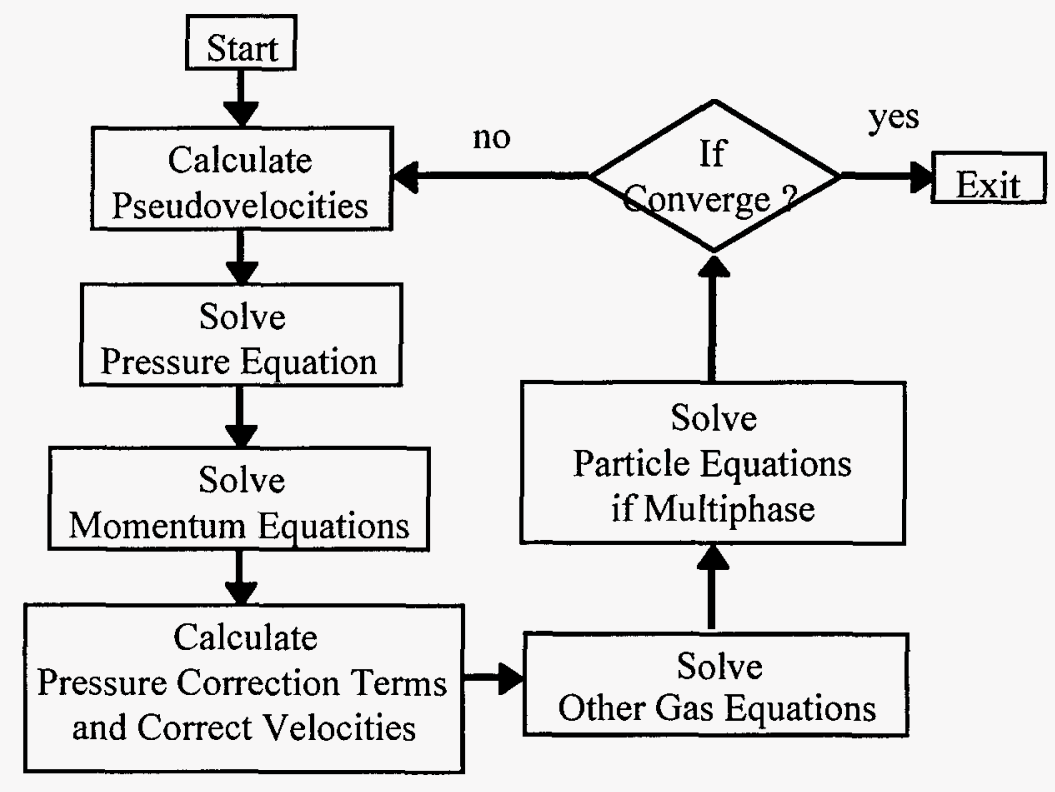

Figure 13 Flow Diagram of Iterative Solution Routine 


\subsubsection{Solution of Discretized Algebraic Equations}

In a two-dimensional grid of $\mathrm{m}$ grid points in the $\mathrm{x}$-direction and $\mathrm{n}$ grid points in the $\mathrm{y}$ direction, a grid point can be identified by a pair of indices $(i, j)$ as shown in Fig. 14. Note that the even points are scalar cell points and the odd points are the interface points. The points at $\mathrm{i}=2$ and $\mathrm{m}$ and $\mathrm{j}=2$ and $\mathrm{n}$ are assumed to be boundary points. Flow properties at the boundary points are known values; for other types of boundary conditions, implementation of the boundary condition eliminates the boundary value of the independent variable from the discretization equation of a computational cell adjacent to a boundary. A general discretized equation, Eq. 4.6, can be rewritten as

$$
d_{i, j} \xi_{i, j}=a_{i, j} \xi_{i, j+2}+b_{i, j} \xi_{i, j-2}+e_{i, j} \xi_{i+2, j}+f_{i, j} \xi_{i-2, j}+g_{i, j} i=4 \text { to } m-2 \text { and } j=4 \text { to } n-2
$$

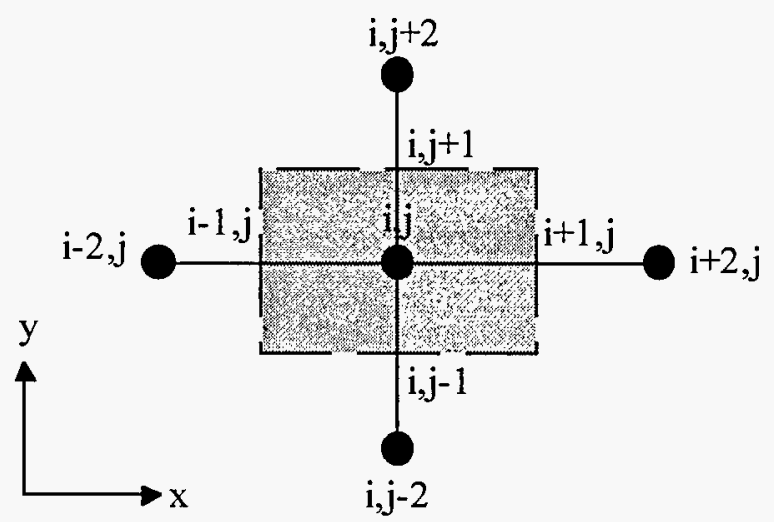

Figure 14 Computational Cell Indexing of a Two-Dimensional Grid

A line-by-line iteration method is used to solve discretized algebraic equations. The method operates on one line, either row or column, of grid cells at a time. To illustrate, consider an $\mathrm{i}^{\text {th }}$ row of cells in the grid. The unknowns in the east and west cells $\left(\xi_{\mathrm{i}+2 \mathrm{j}}\right.$ and $\left.\xi_{\mathrm{i}-2, \mathrm{j}}\right)$ are taken as known from the current estimate. Then Eq. 4.40 can be simplified as

$$
\mathrm{d}_{\mathrm{j}} \xi_{\mathrm{j}}=\mathrm{a}_{\mathrm{j}} \xi_{\mathrm{j}+2}+\mathrm{b}_{\mathrm{j}} \xi_{\mathrm{j}-2}+\mathrm{c}_{\mathrm{j}} \quad \mathrm{j}=4 \text { to } \mathrm{n}-2
$$

Equation 4.41 forms a linear system with a tridiagonal coefficient matrix. An efficient algorithm for solving this system by Gauss elimination is the tridiagonal matrix algorithm. The algorithm can be formulated to work with any of the boundary conditions. Define

$$
\begin{aligned}
& P_{2}=0 \\
& P_{j}=\frac{a_{j}}{d_{j}-b_{j} P_{j-2}} \quad j=4 \text { to } n \\
& Q_{2}=0
\end{aligned}
$$




$$
Q_{j}=\frac{b_{j} Q_{j-2}+c_{j}}{d_{j}-b_{j} P_{j-2}} \quad j=2 \text { to } n
$$

After computing $P_{j}$ and $Q_{j}$ for $j$ from 2 to $n$, the solution is obtained by computing $\xi_{j}$ for $j$ decreasing from $\mathrm{n}-2$ to 4 according to

$$
\xi_{\mathrm{j}}=\mathrm{P}_{\mathrm{j}} \xi_{\mathrm{j}+2}+\mathrm{Q}_{\mathrm{j}} \quad \mathrm{j}=4 \text { to } \mathrm{n}-2
$$

This line-by-line method can be applied to either rows or columns of the computational cells, and the sweep can be applied in either direction. Therefore, all sorts of possibilities become evident in the implementation of an equation solver. For example, alternating-direction (back and forth) sweeps might be assumed to be an excellent choice because the boundary conditions from any particular side of the computational domain would be propagated fairly rapidly to the other side by one of the four possible line-by-line sweeps. In practice, however, an alternating direction has been found not to produce either the most rapid convergence or the best converged solution. Most flow problems have a primary flow direction. In the primary flow direction convection tends to overwhelm diffusion, and even in the cross-stream direction diffusion is usually a secondary effect when compared to convection in the primary flow direction. Therefore, convergence is usually obtained most rapidly if the equation solver sweeps in the primary flow direction. Furthermore, many flow problems are symmetric in the mean flow about the centerline of the primary flow direction. A good check for programming errors is to verify that this symmetry is preserved in a solution computed over the entire flow field, not just from the symmetry line to the outer boundary.

Computational tests showed that symmetry is not only very sensitive to programming errors, but also to the organization of the equation solver. If the equation solver sweeps across lines of symmetry, using updated values of the independent variable, it destroys the symmetry of a symmetric initial estimate used to start the iteration; the asymmetry introduced in this process can become numerically self-preserving, so that even in a reasonably well-converged computation, asymmetries may persist in the fourth and even sometimes the third decimal place for some variables. A broader class of flow problems is nearly symmetric with respect to boundary conditions. For this class of problems, the equation solver should be designed to preserve symmetry in the solution when the boundary conditions are symmetric, so that when computations are done with somewhat asymmetric boundary conditions, the asymmetries that show up in the solution of the interior of the computational domain are not the result of numerically induced asymmetries by the equation solver, but may be properly attributed to the physical asymmetries at the boundaries of either variables or the boundary geometry. 


\section{CODE DESCRIPTION}

Argonne National Laboratory has developed a computer code for the simulation of multiphase reacting flows. The code was written in the FORTRAN language based on the formulation derived in Chapters 3 and 4. The code uses an integral approach to solve for a combustion flow field, so it is called ICOMFLO. ICOMFLO uses a main control program to call subroutines to perform the detailed computations. A subroutine is a collection of FORTRAN instructions to perform a specific function. Table 4 lists the main control program and major subroutines of the ICOMFLO code. The main control program and the subroutines are described in the following sections.

\section{Table 4 Description of ICOMFLO Subroutines}

\begin{tabular}{|l|l|}
\hline \multicolumn{1}{|c|}{ Name } & \multicolumn{1}{c|}{ Description } \\
\hline AOMAIN & main control program \\
\hline ADLBL & linear equation solver using tridiagonal matrix algorithm \\
\hline DEVA & calculates the evaporation rate of the condensed phase when required \\
\hline DFLOW & solves for the particle flow variables \\
\hline DKEL & calculates multiphase turbulent kinetic energy exchange \\
\hline DNDEN & calculates inlet boundary droplet number density in all size groups \\
\hline GFLOW & solves for the gas flow variables \\
\hline GRIDS & defines a computational grid system \\
\hline GSOLVE & solves gas flow governing equations \\
\hline HPRINT & prints reference values and dimensionless parameters \\
\hline INITSV & sets an initial flow field \\
\hline INTP & interpolates and extrapolates flow variables \\
\hline OSAVE & saves system state at previous iteration \\
\hline SAV2F & saves intermediate results for restart \\
\hline SBC & sets steady boundary conditions \\
\hline SETUP & initializes flow properties and numerical parameters \\
\hline SOURCE & calculates source terms for each governing equation \\
\hline SPHASE & $\begin{array}{l}\text { gas-phase flow computation control; also improves initial guess for two- } \\
\text { phase calculations }\end{array}$ \\
\hline SPRINT & prints results of the system state \\
\hline STDY2P & solves steady-state flow field \\
\hline
\end{tabular}

\subsection{Main Control Program}

The main control program, which calls subroutines to do necessary computations, is brief. Its flowchart is shown in Figure 15. As shown in the flowchart, a computational run consists of three major processes: (1) program setup and flow field initialization, (2) flow field calculation, 
and (3) result printout. Variables commonly used in the main control program and the subroutines are included in common blocks. Common variables and their descriptions are listed in Appendix 1. Common blocks and variables are declared in a file called COMMON.BLK. The common block file needs to be included in the main control program and any subroutine that uses common variables. An INCLUDE statement for the common blocks is put in subroutines that use common variables. Program parameters and the flow domain are defined before the flow field is solved iteratively. While solving the flow field, intermediate results may be saved in a restart file, RESTART.D. In a later run the restart file can be used to initialize the flow field and save computational time; it is most useful during the debugging process. After the flow field is solved, results are saved into a data file, RESULT.D, for post processing in separate plotting routines to be developed by a user or available in commercial software packages.

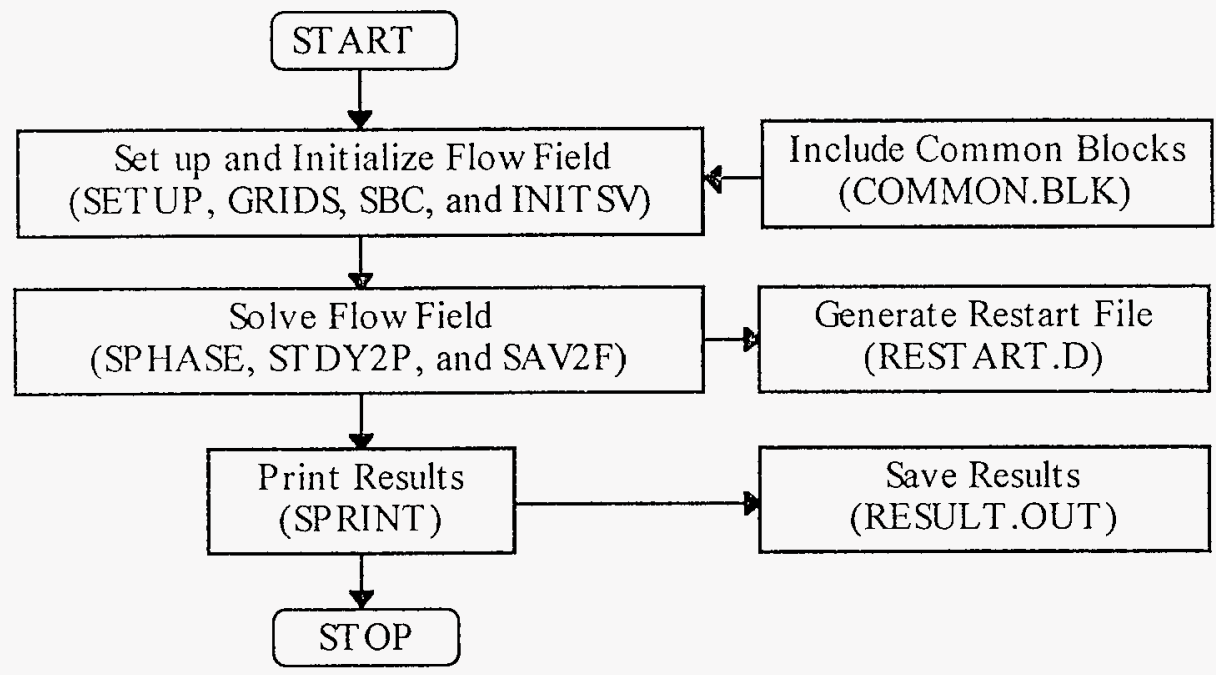

Figure 15. Flowchart of the Main Control Program

\subsection{Setup and Initialization}

Figure 16 shows the flowchart of the setup process. As shown in the flow chart, the setup process includes four steps: (1) assign values to program variables, (2) define a grid for the computational domain, (3) set boundary conditions, and (4) initialize a flow field.

\subsubsection{Subroutine SETUP}

SETUP assigns values to groups of system parameters. The parameter groups include global constants; selection parameters; solution equation indices; chamber geometries; gas, particle, and jet flow conditions; gas properties; turbulence parameters; numerical control parameters; and kinetic constants. These parameters are listed in Tables 5-15. In these tables, the symbol of a system parameter is shown in column 1 , its description in column 2 , and a sample value in column 3. 


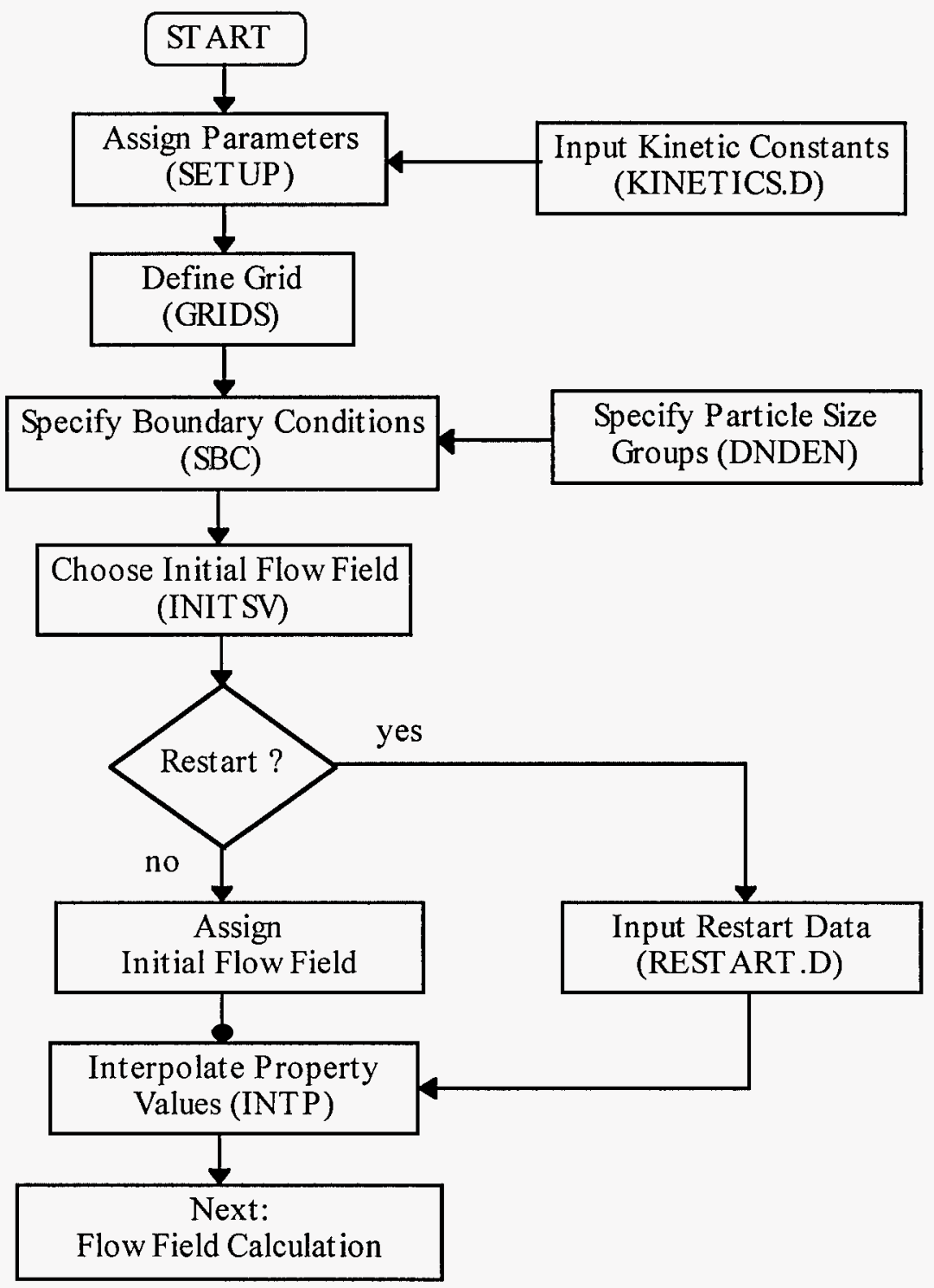

Figure 16 Flowchart of the Setup Process

Table 5 lists global constants. SMALL and BIG are lower and upper limit numbers for numerical calculation; their values may depend on the type of computer used to run the program. RU is the universal gas constant, GRA is the gravitational acceleration constant (with two components, to allow for different orientations of the flow domain in a gravitational field), and the rest are commonly used constants. Note that the values listed in Table 5 should be defined in the program to double precision if the constant is known to double precision (such as $\pi$ ). 
Table 5 List of Global Constants

\begin{tabular}{|l|l|r|}
\hline \multicolumn{1}{|c|}{ Symbol } & \multicolumn{1}{c|}{ Description } & Value \\
\hline BIG & a big number for numerical control & $1 . \mathrm{D} 20$ \\
\hline GRA & gravitational acceleration $(\mathrm{m} / \mathrm{s})$ & 9.80621 \\
\hline ONE & constant & 1 \\
\hline PII & constant $\pi$ & 3.1416 \\
\hline RU & universal gas constant $(\mathrm{J} / \mathrm{kmol} / \mathrm{K})$ & 8314.3 \\
\hline SMALL & a small number for numerical control & $1 . \mathrm{D}-20$ \\
\hline THIRD & constant & 0.3333 \\
\hline ZERO & constant & 0 \\
\hline
\end{tabular}

Table 6 lists selection parameters. IRSTYP is a restart index: 0 for a fresh run and 1 for a restart run. IBAKUP is an index for saving a backup file RESTAR.D: 0 for saving the backup file and 1 for not saving it. CYL is a switch for a computational run to be in a rectangular (FALSE) or a cylindrical (TRUE) coordinate system. NPHAS indicates number of phases (1 or 2) involved in the flow computation. REACT is a switch for a nonreactive (FALSE) or a reactive (TRUE) flow.

Table 6 List of Selection Parameters

\begin{tabular}{|l|l|c|}
\hline \multicolumn{1}{|c|}{ Symbol } & \multicolumn{1}{c|}{ Description } & Value \\
\hline IRSTYP & restart index & $0 / 1$ \\
\hline IBAKUP & backup file index & $0 / 1$ \\
\hline CYL & switch for a rectangular or a cylindrical coordinate & $\mathrm{T} / \mathrm{F}$ \\
\hline NPHAS & number of phases in the flow & $1 / 2$ \\
\hline REACT & switch for a reactive or a nonreactive flow & $\mathrm{T} / \mathrm{F}$ \\
\hline
\end{tabular}

A solution routine is used to solve the governing equations of gas flow properties besides velocity components $(\mathrm{u}, \mathrm{v})$ and pressure (p). Table 7 lists equation solution indices. IYF, IPHI, IH, IK, IEPS, IYIR, IYSV, IYPR, and IYOX are equation indices of fuel $\left(\mathrm{Y}_{\mathrm{f}}\right)$, richness $(\phi)$, enthalpy $(h)$, turbulent kinetic energy $(k)$, turbulent kinetic dissipation rate $(\varepsilon)$, inert gas $\left(Y_{e}\right)$, seed vapor $\left(Y_{s v}\right)$, product $\left(Y_{p}\right)$, and oxidizer $\left(Y_{0}\right)$, respectively. The table also shows a sample set of equation numbers: IYF $=1, \mathrm{IPHI}=2, \mathrm{IH}=3, \mathrm{IK}=4, \mathrm{IEPS}=5, \mathrm{IYIR}=6, \mathrm{IYSV}=7$, $\mathrm{IYPR}=8$, and IYOX $=9$. After assigning equation numbers, a user can select a set of governing equations in the solution routine for a specific flow field. LSTAR and LEND represent the starting and ending points of the equation solution routine, respectively. If LSTAR $=1$ and $\operatorname{LEND}=7$, the solution routines solve equations from 1 to 7 , including equations of fuel species, richness, enthalpy, turbulent kinetic energy, turbulent dissipation rate, inert gas species, and seed vapor. In this case, IYPR and IYOX must still be defined as shown because the equation indices also serve a double role as array indices for storage of variable values. The last differential 
equation that needs to be solved in this case is that for seed vapor (IYSV), because product concentration can be calculated algebraically from the global species conservation equation and oxidizer concentration can also be calculated algebraically from the richness variable values and the fuel concentration.

Table 7 List of Equation Solution Indices

\begin{tabular}{|l|l|c|}
\hline \multicolumn{1}{|c|}{ Symbol } & \multicolumn{1}{c|}{ Description } & Value \\
\hline IEPS & turbulent kinetic dissipation rate & 5 \\
\hline IH & enthalpy & 3 \\
\hline IK & turbulent kinetic energy & 4 \\
\hline IPHI & richness & 2 \\
\hline IYF & fuel & 1 \\
\hline IYIR & inert gas & 6 \\
\hline IYOX & oxidizer & 9 \\
\hline IYPR & product & 8 \\
\hline IYSV & seed vapor (potassium) & 7 \\
\hline LEND & ending point of differential equation solution routine & 7 \\
\hline LSTAR & starting point of differential equation solution routine & 1 \\
\hline
\end{tabular}

Table 8 lists flow chamber geometries. RLE, XLE, and WIDTH are height, length, and width of the flow chamber, respectively. R0 is a reference length used to normalize length scales. The detailed flow domain and the grid system covering it are defined in the GRIDS subroutine (Section 5.2.2).

Table 8 List of Chamber Geometries

\begin{tabular}{|l|l|c|}
\hline \multicolumn{1}{|c|}{ Symbol } & \multicolumn{1}{|c|}{ Description } & Value \\
\hline R0 & reference length $(\mathrm{m})$ & 1.0 \\
\hline RLE & chamber height $(\mathrm{m})$ & 1.0 \\
\hline WIDTH & chamber width $(\mathrm{m})$ & 1.0 \\
\hline XLE & chamber length $(\mathrm{m})$ & 4.7 \\
\hline
\end{tabular}

Table 9 lists inlet gas flow conditions. YF0, YIR0, YSV0, YPR0, and YOX0 are inlet species concentrations, given as mass fractions, of fuel, inert gas, seed vapor, product, and oxidizer, respectively. WF, WIR, WSV, WPR, WOX, WT0 are molecular weights of fuel, inert gas, seed vapor, product, oxidizer, and inlet mixture, respectively. PG0, UG0, and T0 are inlet pressure, velocity, and temperature, respectively. TINF is a reference temperature. STOICH is the stoichiometric mass ratio of oxidizer to fuel. 
Table 9 List of Inlet Gas Flow Conditions

\begin{tabular}{|l|l|r|}
\hline \multicolumn{1}{|c|}{ Symbol } & \multicolumn{1}{|c|}{ Description } & Value \\
\hline YF0 & inlet fuel species concentration & 0.368 \\
\hline YIR0 & inlet inert gas species concentration & 0.39 \\
\hline YSV0 & inlet seed vapor species concentration & 0.0 \\
\hline YPR0 & inlet product species concentration & 0.242 \\
\hline YOX0 & inlet oxidizer species concentration & 0.0 \\
\hline WF & molecular weight of fuel $(\mathrm{kg} / \mathrm{kmol})$ & 28.61 \\
\hline WIR & molecular weight of inert gas $(\mathrm{kg} / \mathrm{kmol})$ & 20.76 \\
\hline WSV & molecular weight of seed vapor $(\mathrm{kg} / \mathrm{kmol})$ & 39.0 \\
\hline WPR & molecular weight of product $(\mathrm{kg} / \mathrm{kmol})$ & 49.37 \\
\hline WOX & molecular weight of oxidizer $(\mathrm{kg} / \mathrm{kmol})$ & 28.0 \\
\hline PG0 & inlet gas pressure $(\mathrm{Pa})$ & 57570 \\
\hline UG0 & inlet gas axial velocity $(\mathrm{m} / \mathrm{s})$ & 27.0 \\
\hline T0 & inlet gas temperature $(\mathrm{K})$ & 1974 \\
\hline TINF & reference temperature $(\mathrm{K})$ & 1974 \\
\hline STOICH & stoichiometric mass ratio of oxidizer to fuel & 1 \\
\hline
\end{tabular}

Table 10 lists gas properties. CPF, CPOX, CPPRO, CPIR, and CPSV are specific heats of fuel, oxidizer, product, inert gas, and seed vapor, respectively. The specific heats are arrays of five elements. Generally, only the first element of an array is used. CP0 is a reference specific heat. GDF0 is mass diffusion coefficient, GL0 is thermal conductivity, and GMU0 is molecular viscosity.

Table 11 lists inlet particle and flow conditions. DDENS is particle density. CL is particle specific heat. TB is boiling temperature, and ELH is latent heat. ACDD, an array of two elements, contains the two coefficients of the empirical particle dispersion function $\varphi$, Eq. 3.16. NT and NS, are parameters $a$ and $b$ of inlet droplet size distribution, Eq. 2.13. TL0 is inlet particle temperature, and ULO is inlet particle velocity. Note that inlet particle temperature and velocity are not required to be constant. Inlet particle temperature and velocity distributions can easily be added in the SBC subroutine, and in these cases UL0 and TLO become simply reference values, most likely the mean of the inlet distribution. RLM0 is mean particle radius.

The ICOMFLO code allows side jets in a combustion chamber. Table 12 lists jet flow parameters. North jet parameters end with an "N" and south jet parameters end with an "S". BETAJN and BETAJS are jet angles. OXWJN and OXWJS are oxygen concentrations of the jets. YFWJN and YFWJS are fuel concentrations of the jets. YIRWJN and YIRWJS are inert gas concentrations of the jets. YPRWJN and YPRWJS are product concentrations of the jets. YSVWJN and YSVWJS are seed vapor concentrations of the jets. TWJN and TWJS are jet temperatures. VJMAGN and VJMAGS are jet mass flow rates. 
Table 10 List of Gas Properties

\begin{tabular}{|l|l|r|}
\hline \multicolumn{1}{|c|}{ Symbol } & \multicolumn{1}{c|}{ Description } & Value \\
\hline CP0 & reference specific heat $(\mathrm{J} / \mathrm{kg})$ & 1241.0 \\
\hline CPF(5) & specific heat of fuel $(\mathrm{J} / \mathrm{kg} / \mathrm{K})$ & 1317.5 \\
\hline CPIR(5) & specific heat of inert gas $(\mathrm{J} / \mathrm{kg} / \mathrm{K})$ & 1040.7 \\
\hline CPOX $(5)$ & specific heat of oxidizer $(\mathrm{J} / \mathrm{kg} / \mathrm{K})$ & 847.0 \\
\hline CPPRO $(5)$ & specific heat of product $(\mathrm{J} / \mathrm{kg} / \mathrm{K})$ & 1119.6 \\
\hline CPSV(5) & specific heat of seed vapor $(\mathrm{J} / \mathrm{kg} / \mathrm{K})$ & 1040.7 \\
\hline GDF0 & mass diffusion coefficient & $0.16 \mathrm{D}-4$ \\
\hline GL0 & thermal conductivity $(\mathrm{W} / \mathrm{m} \mathrm{K})$ & 0.0223 \\
\hline GMU0 & molecular viscosity & $1.7894 \mathrm{D}-5$ \\
\hline
\end{tabular}

Table 11 List of Particle Flow Conditions

\begin{tabular}{|l|l|r|}
\hline \multicolumn{1}{|c|}{ Symbol } & \multicolumn{1}{c|}{ Description } & Value \\
\hline DDENS & particle density $\left(\mathrm{kg} / \mathrm{m}^{3}\right)$ & 1960 \\
\hline CL & particle specific heat $(\mathrm{J} / \mathrm{kg} / \mathrm{K})$ & 2093 \\
\hline TB & boiling temperature $(\mathrm{K})$ & 1595 \\
\hline ELH & latent heat $(\mathrm{J} / \mathrm{kg})$ & $3.527 \mathrm{D} 6$ \\
\hline ACDD $(2)$ & coefficients of empirical particle dispersion function & $0.2,0.006$ \\
\hline NS & parameters of inlet droplet size distribution & 4 \\
\hline NT & parameters of inlet droplet size distribution & 4 \\
\hline RLM0 & mean particle radius $(\mathrm{m})$ & $34 . \mathrm{D}-6$ \\
\hline
\end{tabular}

Table 13 lists turbulence parameters. The empirical constants $\mathrm{CM}, \mathrm{C} 1$, and $\mathrm{C} 2$ are used in Eqs. 3.11 and 3.12. Turbulent viscosity is determined for momentum equations using the $k-\varepsilon$ model described previously and combined with the laminar viscosity to obtain an effective viscosity applicable to the full range of flow conditions. In other equations, the diffusivity of a property is assumed to be a fraction of the effective viscosity for momentum. The fractions are represented by the SIGMA array of 10 elements. For turbulent kinetic energy, SIGMA is 1. For turbulent dissipation rate, SIGMA is 1.3. For other properties, SIGMAs are assumed to be 0.7. CTEA and CTDA are constants for estimation of inlet turbulence kinetic energy and dissipation rate. 
Table 12 List of Jet Flow Parameters

\begin{tabular}{|l|l|c|}
\hline \multicolumn{1}{|c|}{ Symbol } & \multicolumn{1}{c|}{ Description } & Value \\
\hline BETAJN & north jet angle (deg) & 130 \\
\hline BETAJS & south jet angle (deg) & 130 \\
\hline OXWJN & north jet oxidizer concentration & 1 \\
\hline OXWJS & south jet oxidizer concentration & 1 \\
\hline TWJN & north jet temperature (K) & 300 \\
\hline TWJS & south jet temperature (K) & 300 \\
\hline VJMAGN & north jet velocity (m/s) & 304 \\
\hline VJMAGS & south jet velocity (m/s) & 304 \\
\hline YFWJN & north jet fuel concentration & 0 \\
\hline YFWJS & south jet fuel concentration & 0 \\
\hline YIRWJN & north jet inert gas concentration & 0 \\
\hline YIRWJS & south jet inert gas concentration & 0 \\
\hline YPRWJN & north jet product concentration & 0 \\
\hline YPRWJS & south jet product concentration & 0 \\
\hline YSVWJN & north jet seed vapor concentration & 0 \\
\hline YSVWJS & south jet seed vapor concentration & 0 \\
\hline
\end{tabular}

Table 13 List of Turbulence Parameters

\begin{tabular}{|l|l|c|}
\hline \multicolumn{1}{|c|}{ Symbol } & \multicolumn{1}{|c|}{ Description } & Value \\
\hline CM & empirical constant for turbulence equations & 0.09 \\
\hline C1 & empirical constant for turbulence equations & 1.44 \\
\hline C2 & empirical constant for turbulence equations & 1.92 \\
\hline CTEA & constant for estimation of inlet turbulence & 0.03 \\
\hline CTDA & constant for estimation of inlet turbulence & 1.0 \\
\hline SIGMA(10) & $\begin{array}{l}\text { ratio of diffusivity to turbulent viscosity for a variable } \\
\text { (exceptions noted above) }\end{array}$ & 0.7 \\
\hline
\end{tabular}

Table 14 lists numerical control parameters. RF and RFC are arrays of relaxation factors and their complementary values. The values listed in the table are the normal values used unless a numerical stability problem arises in computations with a particular set of conditions; then, one or more of the sensitive variables may be relaxed to a greater extent. NTIMES is an array of the number of iterations executed for solving each governing equation in the base-level equation solver (subroutine ADLBL) in the course of each global iteration through the equation of each phase. BGCON and BSCON are convergence criteria based on average mass residuals for gas and particle phases. Note that mass residual is normalized by inlet mass flow rate. MAXGI and MAXSI are maximum numbers of iterations for gas- and particle-phase calculations if they do not converge for single- and two-phase applications, respectively. In two-phase applications, 
MAXGI becomes the maximum number of gas-phase iterations performed to yield an estimation of the gas flow field before beginning full two-phase computations. In two-phase computations, several condensed-phase iterations may be performed between each gas-phase iteration. The number of these consecutive condensed-phase iterations is determined by a local variable, IDIT, defined in the steady-state two-phase control subroutine, STDY2P. IDIT is normally set to 2.

Table 14 List of Numerical Control Parameters

\begin{tabular}{|l|l|r|}
\hline \multicolumn{1}{|c|}{ Symbol } & \multicolumn{1}{c|}{ Description } & Value \\
\hline BGCON & convergence criteria for gas phase & $1 . D-10$ \\
\hline BSCON & convergence criteria for particle phase & $1 . D-7$ \\
\hline MAXGI & maximal number of iterations for gas-phase calculation & 200 \\
\hline MAXSI & $\begin{array}{l}\text { maximal number of iterations for particle-phase calcula- } \\
\text { tion }\end{array}$ & 1000 \\
\hline NTIMES(20) & number of iterations for solving each governing equation & 5 \\
\hline RF(21) & relaxation factor & 0.7 \\
\hline RFC $(21)$ & complementary relaxation factor & 0.3 \\
\hline
\end{tabular}

Kinetic constants are determined from a separate kinetics calculation and are input from a data file, KINETICS.D. Table 15 lists these kinetic parameters. RTIM is a reaction time array and DELR is an array of the extent of reaction. DELRT and HRT are arrays of the extent of reaction and enthalpy versus reaction time. TIMED and HRD are arrays of reaction time and enthalpy versus the extent of reaction.

\section{Table 15 List of Empirical Kinetic Constants}

\begin{tabular}{|l|l|c|}
\hline \multicolumn{1}{|c|}{ Symbol } & \multicolumn{1}{|c|}{ Description } & Value \\
\hline RTIM(100) & kinetic reaction time scale $(\mu \mathrm{s})$ & input \\
\hline TIMED $(100)$ & $\begin{array}{l}\text { kinetic reaction time based on the extent of reaction } \\
(\mu \mathrm{s})\end{array}$ & input \\
\hline HRT $(100)$ & enthalpy based on reaction time $(\mathrm{J} / \mathrm{kg})$ & input \\
\hline HRD $(100)$ & enthalpy based on the extent of reaction $(\mathrm{J} / \mathrm{kg})$ & input \\
\hline DELRT $(100)$ & extent of reaction based on reaction time & input \\
\hline DELR $(100)$ & extent of reaction & input \\
\hline
\end{tabular}

\subsubsection{Subroutine GRIDS}

GRIDS defines the computational grid for a flow field. Table 16 lists the GRIDS parameters. $\mathrm{X}$ is an array of $\mathrm{x}$-coordinates for computational nodes and DX is an array of $\mathrm{x}$-cell sizes. MM is the number of $\mathrm{X}$ and DX array elements and is defined in COMMON.BLK. Similarly, $\mathrm{R}$ is an array of $\mathrm{y}$-coordinates for computational nodes and $\mathrm{DR}$ is an array of $\mathrm{y}$-cell 
sizes. $\mathrm{NN}$ is the number of $\mathrm{R}$ and $\mathrm{DR}$ array elements and is defined in COMMON.BLK. IBCELL is a matrix of blocked cell indices. An IBCELL value is assigned to each computational node. If IBCELL is 1 , the cell is blocked; otherwise, it is open to flow through the sides not adjacent to blocked cells.

Table 16 List of Grid Parameters

\begin{tabular}{|l|l|c|}
\hline \multicolumn{1}{|c|}{ Symbol } & \multicolumn{1}{|c|}{ Description } & Value \\
\hline DR(NN) & y-cell size $(\mathrm{m})$ & assigned \\
\hline DRD(ND) & normalized droplet size differential & assigned \\
\hline DX(MM) & x-cell size $(\mathrm{m})$ & assigned \\
\hline IBCELL(MM,NN) & blocked cell index, 0: open cell 1: blocked cell & assigned \\
\hline MM & number of x-nodes & 114 \\
\hline ND & number of droplet size groups & 5 \\
\hline NN & number of y-nodes & 64 \\
\hline R(NN) & y-coordinate of a grid point $(\mathrm{m})$ & assigned \\
\hline RD(ND) & normalized droplet size & assigned \\
\hline $\mathrm{X}(\mathrm{MM})$ & x-coordinate of a grid point $(\mathrm{m})$ & assigned \\
\hline
\end{tabular}

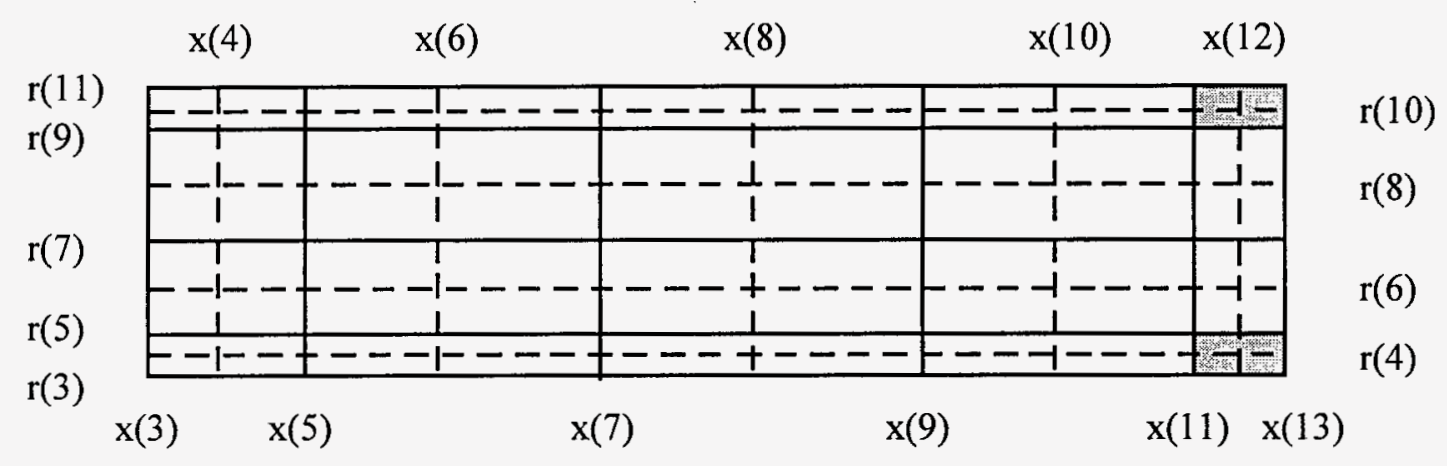

Figure 17 A Sample Grid System with Blocked Cells

A grid includes arrays of $x$ - and $y$-nodes as shown in Figure 17. Coordinates of grid lines are assigned in the $\mathrm{X}$ and $\mathrm{Y}$ arrays. Grid points occur at the intersections of grid lines. Because of the staggered nature of the grid cells for gas-phase scalar variables and $\mathrm{x}$ - and $\mathrm{y}$-direction momentum, the differential equations are not solved at all grid points, but only at the reference points in their particular type of cells. Even-numbered point pairs, i.e., $x(4), \ldots, x(12), r(4), \ldots$, and $\mathrm{r}(10)$, represent coordinates of scalar cell centers (dashed line intersections), and odd-numbered points, i.e., $x(3), \ldots, x(13), r(3), \ldots$, and $r(11)$, represent scalar cell boundaries (solid lines). Cell size arrays DX and DR are calculated from the $\mathrm{X}$ and $\mathrm{Y}$ arrays. To represent a complex flow geometry, some computational cells can be blocked (shaded areas). 
GRIDS also defines droplet size distribution parameters, $\mathrm{RD}$ and $\mathrm{DRD}$. $\mathrm{RD}$ is an array of particle or droplet sizes for ND size groups. DRD is an array of mean droplet size change between a size group and the next larger size group. The number of droplet size groups, ND, is assigned in the common block file.

\subsubsection{Subroutine SBC}

SBC sets steady-state boundary conditions. The boundaries generally include inlet, solid walls, and exit. At the inlet, values of primary flow properties are specified by the user, and those of other flow properties are derived from the primary ones. Primary properties include gas velocity components $(\mathrm{U}$ and $\mathrm{V})$; an absolute reference pressure $\left(\mathrm{P}_{\mathrm{ref}}\right)$, usually specified at the center of the main inlet; temperature (T); concentrations of fuel, oxidizer, product, and seed vapor (GF:IYF, IYOX, IYPR, IYSV); turbulent kinetic energy (GF:IK); turbulent dissipation rate (GF:IEPS); particle velocity components (DU and DV); particle number density (DN); and particle temperature (DT). Derived properties include mixture molecular weight (WT0), density (DNST), inert gas concentration (GF:IYIR), richness (GF:IPHI), gas enthalpy (GF:IH), turbulent viscosity (TMU), gas conductivity (GLAM), gas specific heat (GCP), turbulent diffusivity (GAMA), and void fraction (THETA). For a two-phase flow calculation, particle properties are specified for each of the ND size groups. The DNDEN subroutine is called to calculate initial particle number density in ND size groups, based on Eq. 2.13. Inlet boundaries include wall jets. At a solid wall, gas velocity components are set to zero and most other properties are set equal to the neighboring flow cell (a zero slope condition determined from the solution at one interior point). In some cases, a zero slope wall condition is set by fitting a parabola, using the two nearest interior solution points. At the exit, flow properties are computed from the upstream flow properties, based on an assumed small property slope change (e.g., free flow). The exit flow properties need not be specified. Patankar [27] provides a very detailed discussion of handling outflow boundary conditions. The only difference between his approach and that used here is that a slight slope in outflow boundary velocities is allowed and is computed from a global mass balance calculated by numerically integrating the mass flow across all boundary openings. This enhancement allows the computational domain to be shorter than that required to reach a fully developed flow condition, which is frequently not feasible in practice (especially in reacting flows).

\subsubsection{Subroutine INITSV}

Before solving flow properties, INITSV makes an initial estimation of the results. If a calculation (or run) is a new one, INITSV assumes a simple flow field. Typically, the inlet flow properties are used for all computational nodes. The flow properties include U, V, T, DNST, GF, THETA, DT, DN, DU, and evaporation rate EVP. Property values are assigned only at computational nodes, odd nodes for velocity and even nodes for the other properties. A general property function GF represents species concentrations (IYF, IYOX, IYPR, IYIR, and IYSV), richness (IPHI), enthalpy (IH), and turbulence parameters (IK and IEPS). If there is a solution stored in a restart file, RESTART.D, from a previous run, and the user wishes to begin or resume 
computing from the solution data stored in that file, the parameter IRSTYP is set to 2 and INITSV inputs values of flow properties from the file. (Note that the boundary conditions are not required to be the same as those used to generate the solution stored in the restart file, and therefore the restart file might provide an excellent initial solution estimate for a new problem with boundary conditions only slightly different from those used to obtain the solution saved in the restart file.) After the assigning or inputting of property values to the computational nodes, subroutine INTP is called to calculate property values in noncalculation nodes by interpolation and subroutine DFBC is called to extrapolate particle flow properties to the boundary nodes.

\subsubsection{Subroutine HPRINT}

After INITSV, subroutine HPRINT is called in the main program to print headings, reference values, and dimensionless parameters. Reference values include gas velocity UG0, solid velocity UL0, gas temperature T0, pressure PG0, mean particle size RLM0, particle number density DND0, and particle temperature TL0. Dimensionless parameters include Reynolds number, Schmidt number, group combustion number GC, Eckert number, Damkholer number, fuel/air ratio, Euler number, and Nusselt number DNULO.

\subsection{Flow Field Calculation}

After the setup and initialization step, ICOMFLO solves the governing equations of flow properties. Figure 18 shows a flowchart of the flow field calculation process. For a multiphase flow calculation, subroutines STDY2P, GFLOW, GSOLVE, and DFLOW are used. For a single-phase flow calculation, subroutines SPHASE, GFLOW, and GSOLVE are used.

\subsubsection{Subroutine STDY2P}

STDY2P uses subroutines GFLOW, DFLOW, DEVA, SFLOW, and SAV2F to solve flow properties of both gas and condensed phases. GFLOW updates gas-phase parameters and calls GSOLVE to solve for gas flow properties. A gas-flow calculation is considered converged when mass residue calculated from the continuity equation (or pressure equation) is less than a prescribed convergence limit, BGCON. In a separate iteration routine, DFLOW, DEVA, and SFLOW are used to solve condensed flow properties. DFLOW solves condensed flow properties; DEVA calculates the evaporation rate of the condensed phase if necessary; and SFLOW calculates particle deposition rate on the walls. A condensed flow calculation is considered converged when mass residue calculated from the condensed-phase continuity equation is less than a prescribed convergent limit, BSCON. A solution is obtained when both gas- and condensed-phase calculations converge; otherwise, gas and condensed flow calculations continue until MAXSI iterations are reached. In either case, if the restart backup indicator, IBAKUP1, is set greater than zero, SAV2F is called to save a restart file, RESTART.D. 


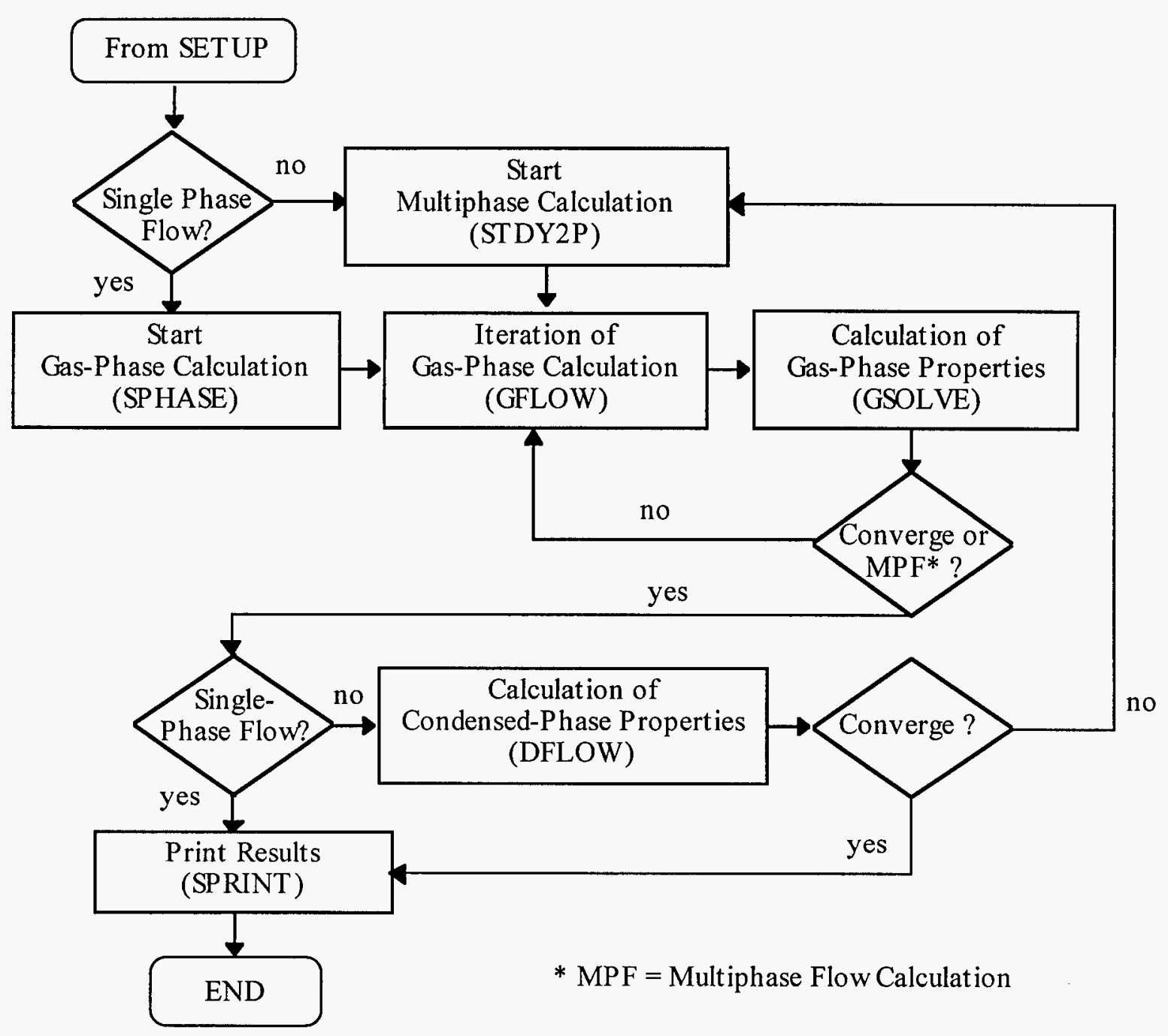

Figure 18 Flowchart of the Flow Calculation Process

\subsubsection{Subroutine SPHASE}

SPHASE sets up an iteration routine to solve for the gas flow field. In the iteration routine, GFLOW is used to solve for gas flow properties. The iteration ends when the calculation converges (i.e., mass residual is less than BGCON) or the iteration number reaches a pre-set value, MAXGI. In either case, before the run ends the current state of the computation is saved in the file RESTART.D if the backup indicator is set so that the computation may be restarted at a later time from the current solution.

\subsubsection{Subroutine GFLOW}

Gas flow properties are solved as follows. First, transport properties (e.g., turbulent viscosity TMU, gas conductivity GLAM, gas specific heat GCP, turbulent diffusivity GAMA) are computed. Next, gas flow properties, including velocity components $U$ and $V$, pressure $P$, fuel 
concentration GF(IYF), enthalpy GF(IH), temperature $T$, turbulent kinetic energy GF(IK), turbulent dissipation rate GF(IEPS), richness GF(IPHI), inert gas concentration GF(IYIR), and seed vapor concentration GF(IYSV), are solved. Those properties at computational nodes are solved in GSOLVE and those at noncomputational nodes are determined in INTP. Computational nodes for scalar properties are even nodes, and those for velocity components are odd nodes. After solving for the flow properties, gas density is calculated from the state equation of a perfect gas, Eq. 2.9.

\subsubsection{Subroutine GSOLVE}

The SIMPLER algorithm described in Section 4.4.3 is used to solve governing equations of gas flow properties. Velocities and pressure are solved first, followed by scalar properties like species concentrations, turbulence parameters, and enthalpy. For each flow property, a governing equation is derived in Chapter 4. All governing equations share the same convective and diffusive forms but they have different source terms. The common convective and diffusive terms are defined in GSOLVE and the source terms are given in the subroutine SOURCE. For each property, the governing equation is solved by assuming that all other properties are known. A linear equation solver, ADLBL, is used to solve the governing equations. ADLBL uses a tridiagonal matrix algorithm (described in Section 4.4.4). Only property values at computational nodes are solved. Once gas enthalpy is obtained, gas temperature is calculated from the gas-phase caloric equation of state, Eq 2.10.

\subsubsection{Subroutine DFLOW}

Particle flow properties are solved in DFLOW. Particle number density is solved first, followed by velocities and temperature. Each flow property is solved for each size group, and there are 'ND' of them. Again, governing equations use the same convective and diffusive forms but have different source terms. The common convective and diffusive terms are defined in DFLOW and the source terms are given in the subroutine SOURCE. A governing equation of a particle flow property for a size group is solved by assuming that all other properties are known and using as values the estimates from the current or previous iteration, depending on the order of solution. If updated values are available, they are used; if not, the values from the previous iteration that remain in the variable storage arrays are used. ADLBL is used to solve the linearized governing equations by solving a whole grid row or column at a time, using the tridiagonal matrix algorithm and sweeping over the grid as previously discussed.

\subsection{Results and Printout}

SPRINT prints computational results. The results include gas pressure $\mathrm{P}$, temperature $\mathrm{T}$, density DNST, velocities U and V, species concentrations of fuel, oxidizer, product, inert gas, and seed vapor, turbulent viscosity TMU, turbulent kinetic energy, turbulent dissipation rate, enthalpy, particle number density DN, particle velocity components DU and DV, and particle temperature DT. 


\section{SAMPLE CALCULATION AND CODE VALIDATION}

A sample problem will illustrate the use of the ICOMFLO computer code for a flow field calculation. The sample problem is the multiphase reacting flow in a TRW second-stage combustor for MHD power generation [21,22]. Figure 19 shows a simple sketch of a twodimensional straight channel representing the TRW combustor. The combustor consists of an inlet, an exit, and two injectors on two side walls. The inlet flow includes a stream of coal gas and seed (potassium) particles. Coal gas is the product of substoichiometric coal-air combustion in a first-stage swirl combustor. Seed material, which ionizes at second-stage combustor temperatures, is added to make a plasma flow. Two streams of oxidizer are injected from side walls into the coal gas flow to complete the combustion and further raise the gas temperature. The injection is made at an angle $\theta$ to enhance combustion and fluid mixing. Due to the high temperature of the combustion, the potassium particles are vaporized and the potassium atoms are ionized. The exit flow is a plasma flow containing ionized seed vapor and combustion products. The exit flow is sent to an MHD channel for electric power generation. The ICOMFLO computer code is used to simulate the combustion flow system and calculate flow properties (i.e., pressure, temperature, density, velocities, species concentrations, etc.) at grid points covering the entire domain of the combustor. The extensive amount of data generated allows post-processing of the data to produce a complete picture of processes occurring within the combustor.

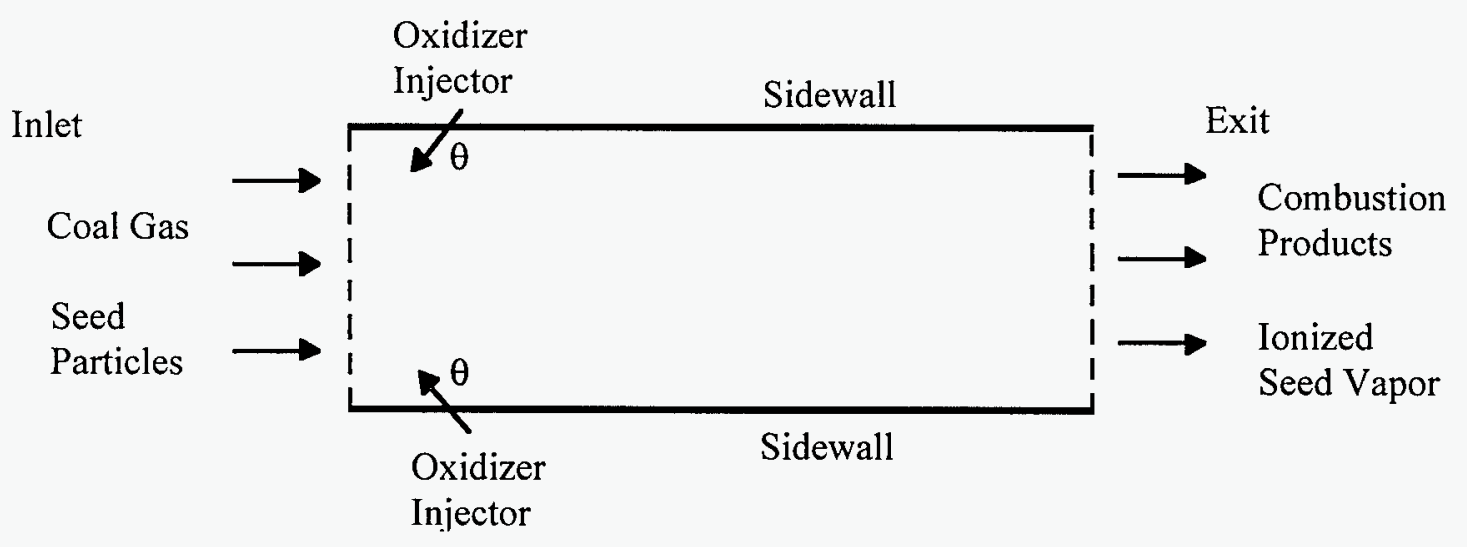

Figure 19 TRW's MHD Second-Stage Combustor

\subsection{Parameter Assignment}

The ICOMFLO code was written for a general multiphase reacting flow. Flow configurations, boundary conditions, and reaction mechanisms of the sample reacting flow need to be specified in the ICOMFLO code before performing a calculation. A kinetic constant file KINETIC.D for the coal gas combustion process was obtained from a separate kinetics calculation. Flow and numerical variables are initialized in the SETUP subroutine. A computational grid for the TRW MHD second-stage combustor was defined in the GRIDS subroutine. Conditions of boundary flows were defined in the SBC subroutine. 
A kinetics calculation of the coal gas combustion was performed as described in Section 3.1. The time-dependent extent of reaction DELRT and heat of combustion HRT were computed using NASA's kinetics computer code. Some computed results are shown in Table 17. In the table, DELRT and HRT are tabulated versus the reaction time RTIM. After a reaction time of $0.25 \mu \mathrm{s}$, the extent of reaction is 0.3926 and the accumulated heat of combustion is 30.36 $\mathrm{kcal} / \mathrm{mol}$. After a reaction time of $4.995 \mu \mathrm{s}$, the combustion is nearly complete: the extent of reaction is 0.5678 and the heat of combustion is $55.93 \mathrm{kcal} / \mathrm{mol}$. In calculating the source terms for gas enthalpy, the extent of reaction is sometimes treated as an independent variable in place of the reaction time. Computed kinetic results were compiled and saved into a data file KINETIC.D, including 100 data points of computed extent of reaction DELRT and accumulated heat of combustion HRT versus reaction time RTIM and another 100 data points of computed accumulated heat of combustion HRD and reaction time TIMED versus the extent of reaction DELR.

Table 17 Computed Kinetic Constants

\begin{tabular}{|c|c|c|}
\hline RTIM & DELRT & HRT \\
\hline .2500 & .3926 & 30.0360 \\
\hline .5000 & .4228 & 41.3807 \\
\hline 1.0000 & .4706 & 49.5504 \\
\hline 2.0000 & .5278 & 54.1965 \\
\hline 4.9500 & .5678 & 55.9324 \\
\hline
\end{tabular}

The number of kinetic data points NR is assigned in a common block file COMMON.BLK. A sample common block file is shown in Appendix 4. The number of scalar $\mathrm{x}$ - and y-nodes MZ and NZ, and the number of particle size groups ND, are also assigned in this file. Including the momentum nodes, actual $\mathrm{x}$ - and $\mathrm{y}$-nodes $\mathrm{MM}$ and $\mathrm{NN}$ are double those of $\mathrm{MZ}$ and NZ. Values of these common block parameters for the sample calculation are listed below:

$\begin{array}{ll}\text { MZ } & 57 \\ \text { MM } & 114 \\ \text { NZ } & 32 \\ \text { NN } & 64 \\ \text { ND } & 5 \\ \text { NR } & 100\end{array}$


Table 18 Sample Values of SETUP Parameters

\begin{tabular}{|l|r|l|r|l|r|}
\hline Parameter & \multicolumn{1}{|c|}{ Value } & Parameter & \multicolumn{1}{c|}{ Value } & Parameter & Value \\
\hline NPHAS & 2 & WOX & 28.0 & TWJN & 300 \\
\hline REACT & T & GDF0 & $0.16 \mathrm{D}-4$ & TWJS & 300 \\
\hline IRSTYP & 0 & GL0 & 0.0223 & VJMAGN & 304 \\
\hline IBAKUP & 1 & GMU0 & 1.7894 D-5 & VJMAGS & 304 \\
\hline BGCON & $1 . D-10$ & STOICH & 1 & YFWJN & 0 \\
\hline BSCON & $1 . D-7$ & PG0 & 57570 & YFWJS & 0 \\
\hline MAXGI & 200 & UG0 & 27.0 & YIRWJN & 0 \\
\hline MAXSI & 1000 & T0 & 1974 & YIRWJS & 0 \\
\hline CPF(1) & 1317.5 & YF0 & 0.368 & YPRWJN & 0 \\
\hline CPIR(1) & 1040.7 & YIR0 & 0.39 & YPRWJS & 0 \\
\hline CPOX(1) & 847.0 & YSV0 & 0.0 & YSVWJN & 0 \\
\hline CPPRO(1) & 1119.6 & YPR0 & 0.242 & YSVWJS & 0 \\
\hline CPSV(1) & 1040.7 & YOX0 & 0.0 & DDENS & 1960 \\
\hline WF & 28.61 & BETAJN & 130 & CL & 2093 \\
\hline WIR & 20.76 & BETAJS & 130 & TB & 1595 \\
\hline WSV & 39.0 & OXWJN & 1 & RLM0 & $34 . D-6$ \\
\hline WPR & 49.37 & OXWJS & 1 & & \\
\hline
\end{tabular}

Subroutine SETUP assigns values to parameters of the flow system. Major parameter groups are selection parameters; differential equation and variable indices; chamber geometries; gas, particle, and jet flow conditions; gas properties; turbulence parameters; numerical control parameters; and kinetic constants. Table 18 shows sample values of major SETUP parameters. Kinetic data are read in from the KINETIC.D file.

Subroutine GRIDS was written to define a computational grid for the reacting flow field of the MHD second-stage combustor as shown in Fig. 19. The grid for the sample problem is shown in Fig. 20. It has 57 scalar nodes in the $x$-direction and 32 nodes in the $y$-direction. Dense grid point spacing was used near the injection areas, where steep flow property changes are expected.

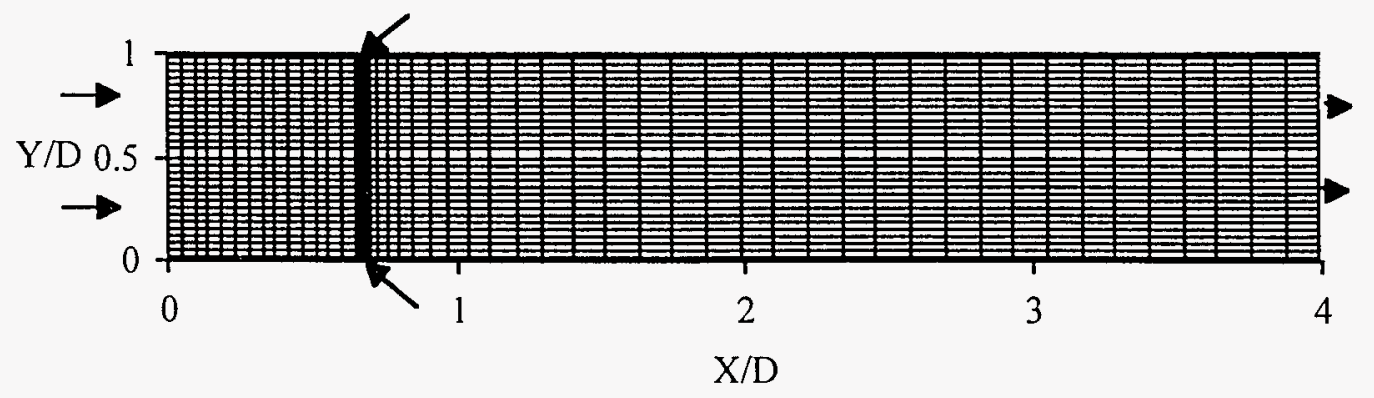

Figure 20 Grid System for the Sample Combustor 
Subroutine SBC sets boundary flow conditions of the sample problem. The boundary flow includes an inlet stream and two wall jets (north and south). The inlet stream consists of coal gas (fuel, product, and inert gas) and seed particles (five particle size groups). The jets consist of only oxidizer. Uniform velocity and temperature were assumed on the boundaries.

\subsection{Numerical Iteration}

After precalculation setup, the ICOMFLO code is ready for the flow field calculation. The calculation can be performed on a 486/Pentium personal computer using Microsoft FORTRAN Power Station software. First, the main control program and all the subroutines are copied to a subdirectory (i.e., C:IMHDIREPORT1\PRM). Table 19 lists the names of the ICOMFLO files. These files contain source listings of the FORTRAN programs.

Table 19. List of the ICOMFLO Files

\begin{tabular}{|llllll|}
\hline \multicolumn{2}{|l}{$\begin{array}{l}\text { Volume in drive } \text { C has no label } \\
\text { Directory of }\end{array}$ C: $\backslash$ MHD $\backslash$ REPORTI $\backslash$ PRM } & \\
AOMAIN & FOR & DNDEN & FOR & SBC & FOR \\
ADLBL & FOR & FUELX & FOR & SETUP & FOR \\
ADTIME & FOR & GELOW & FOR & SFLOW & FOR \\
BC & FOR & GRIDS & FOR & SOURCE & FOR \\
CONC & FOR & GSOLVE & FOR & SPHASE & FOR \\
DA & FOR & HPRINT & FOR & SPRINT & FOR \\
DEVA & FOR & INITSV & FOR & STDY2P & FOR \\
DFBC & FOR & INTP & FOR & TDPRIN & FOR \\
DELOW & FOR & OSAVE & FOR & TSTEP & FOR \\
DKEL & FOR & SAV2E & FOR & & \\
\hline
\end{tabular}

Next, the FORTRAN programs are compiled and linked together to generate an execution program (i.e., MHD.EXE). The steps needed to build a project and to compile, link, and execute the program are described in the user's guide for Microsoft FORTRAN PowerStation [28]. Running MHD.EXE starts a flow calculation.

During computation, some flow field results are printed out for debugging purposes. A sample printout is shown in Appendix 4. The printout includes summaries of inlet flow properties, grid assignment, reference and dimensionless parameters, and typical iteration results for various flow field variables. After each iteration of the gas flow field calculation, average and maximal mass residuals (AVEB and SMX), the indices of the node of the maximal mass residual (IMX, JMX), and velocity components (U and V), pressure (P), density (DNST), and temperature $(\mathrm{T})$ at the location of the maximal mass residual are printed. The printout values are all dimensionless. The reference mass residual is the inlet mass flow rate; reference velocity is the 
inlet gas velocity (UG0); reference pressure is the inlet gas pressure (PG0); reference density is the inlet gas density (DNST0); and reference temperature is the inlet gas temperature (T0), unless an inlet distribution is defined. (Then, these reference values are normally assigned the mean of the inlet distribution.)

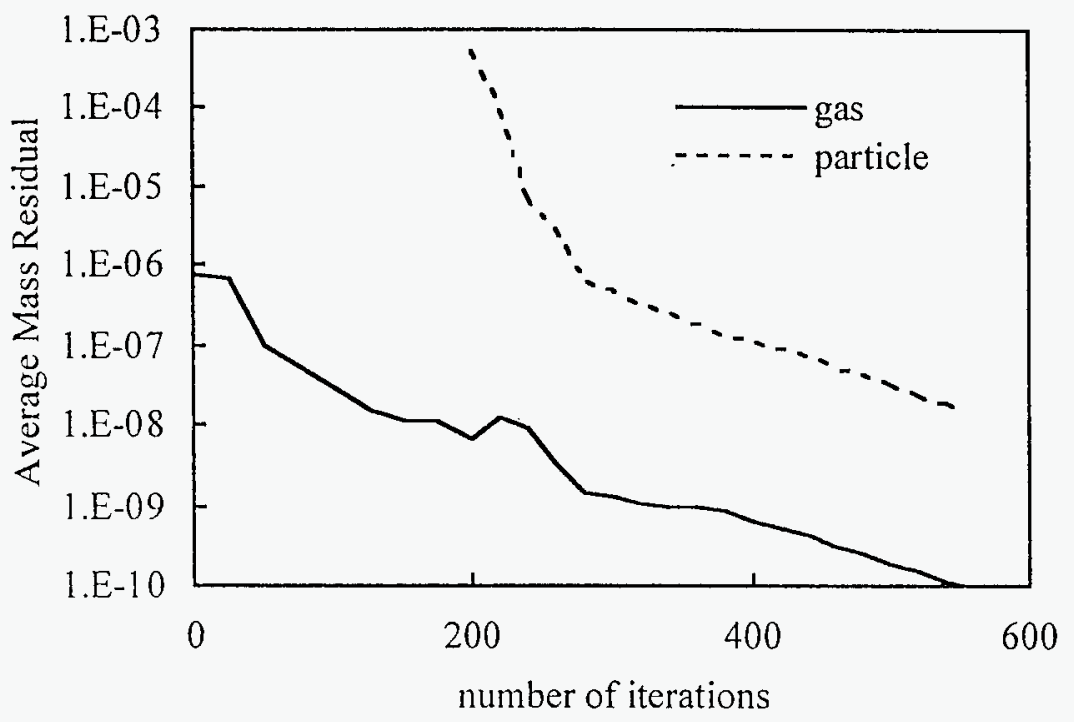

Figure 21 Numerical Convergence of a Sample Calculation

In a typical computation, the mass residual of each cell in the computational domain is checked after each iteration of solving the governing equation set. The mass residual is required to be smaller than a preset convergence criterion before stopping the iteration. If the process converges, mass residual becomes smaller as the iteration goes on. To avoid numerical divergence of the condensed-phase calculations, 200 iterations of the gas flow field calculation were executed before beginning two-phase flow field calculations. Appendix 4 shows printouts of the first and the 200th iterations. After 200 iterations, the average gas mass residual taken over all computational cells improved from $7.598 \times 10^{-7}$ at the first iteration to $7.02 \times 10^{-9}$. Note also that the average mass residual usually degrades or improves very slowly in early iterations, because an initial guess of a completely uniform flow field satisfies mass conservation trivially in nearly all computational cells. Also, although the solution algorithm provides fairly good results after a only a few iterations, starting up a full two-phase flow calculation without first computing a fairly good solution for the gas-phase flow pattern may lead to divergence of the computation. Following the gas flow calculation, iterations of the two-phase flow field calculation are performed until the solution converges (i.e., gas mass residual is less than BGCON and particle residual is less than BSCON). Each two-phase iteration includes a gas flow field calculation and two particle flow calculations. Similarly, after one iteration of the particle flow field calculation, average and maximal mass residuals (AVEB and SMX), the indices of the node of the maximal mass residual (IMX, JMX), and velocity components (UL and VL), pressure (P), particle void fraction (THETAC), median size particle number density (DN), temperature (TL), and evaporation rate (EVP) of the location of the maximal mass residual and a global particle mass 
balance are printed. This sample calculation converges after 352 iterations of two-phase flow calculation. Appendix 4 shows printouts of the first and the last iterations. After the first iteration of the two-phase flow field calculation, average gas mass residual is $6.921 \times 10^{-9}$ and average particle mass residual is $5.259 \times 10^{-4}$. At the end, average gas and particle mass residuals are improved to $9.914 \times 10^{-11}$ and $1.595 \times 10^{-8}$, respectively.

Figure 21 shows average gas and particle mass residuals versus the number of iterations. After 552 iterations, gas mass residual improves by four orders of magnitude, from $10^{-6}$ to $10^{-10}$. Particle flow field starts to be computed after 200 gas flow calculations and two particle flow calculations are performed for each two-phase flow field iteration. After 704 particle flow calculations, particle mass residual also improves by four orders of magnitude, from $10^{-3}$ to $10^{-7}$. Note that the gas-phase solution degrades after the full two-phase computation is begun at iteration 200. Because of the coupling between the phases, the gas-phase solution with particles is not the same as the solution with particles in the flow, which accounts for the temporary growth of gas-phase mass residual at iteration 200 before it starts downward again.

\subsection{Computational Results}

This sample calculation takes about 90 minutes on a Pentium-90 personal computer. Computed results include gas flow properties (pressure, temperature, density, $\mathrm{x}$-velocity, $\mathrm{y}$-velocity, fuel concentration, oxygen concentration, product concentration, inert gas concentration, seed vapor concentration, turbulent viscosity, turbulent kinetic energy, turbulent dissipation rate, enthalpy, and reaction rate) and particle flow properties (total particle number density, number density, $x$-velocity, $y$-velocity, and temperature of each particle size group, particle void fraction, and evaporation rate). There are five particle size groups: $0.175,0.525,0.875,1.225$, and 1.575 (reference particle size is $68 \mu \mathrm{m}$ ).

ICOMFLO saves the computational results in a file SPRINT.OUT. The results are arranged in a matrix format similar to the grid matrix: 57 rows and 32 columns. Due to the pagesize limitation, the 32 column matrix is divided into three smaller matrices: 57 by 11,57 by 11 , and 57 by 10 columns. In each matrix, the first row gives the $y$-grid coordinates and the first element of a row represents the corresponding $\mathrm{x}$-coordinate. Computed gas temperatures are tabulated in the matrices as shown in Appendix 5. The temperature is shown in a dimensionless form. Multiplying a dimensionless temperature by the reference temperature yields the actual temperature. The reference temperature is $1974 \mathrm{~K}$.

Figure 22 shows results of the sample calculation of the flow field in the combustor with 130-degree oxidizer jet injection. The first three frames - (a), (b), and (c) - are contour plots of temperature, fuel mass fraction, and oxidizer mass fraction, respectively. Temperature is normalized by the inlet temperature of the main flow. The equilibrium temperature of the reaction under adiabatic conditions is about 1.5 times the main flow inlet temperature. The jet injection ports are located at $\mathrm{X} / \mathrm{D}=0.66$. With 130 -degree counter-flow injection, intense mixing occurs 
ports are located at $X / D=0.66$. With 130-degree counter-flow injection, intense mixing occurs upstream of the injectors, and the flame is established in this intense mixing zone. Because the jets penetrate deeply into the main flow, large vortices form behind the jets (Fig. 22d), and the flame follows the vortex boundary from upstream, where fuel and oxidizer first meet, around toward the chamber center as the oxidizer jets are turned downstream. The flame then continues to develop as a diffusion flame in the mixing layer between fuel and oxidizer downstream. In Fig. 22, frames (b) and (c) show that much of the fuel and oxidizer are consumed by the exit plane, and frame (a) shows that the fluid temperature at the exit plane is relatively uniform, approaching the reaction equilibrium temperature.

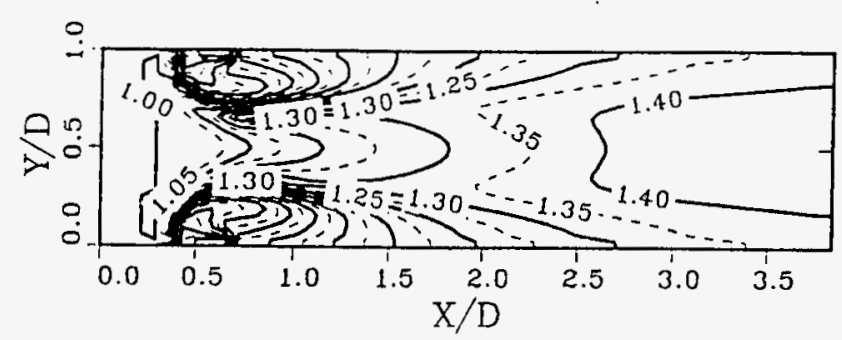

(a) Temperature

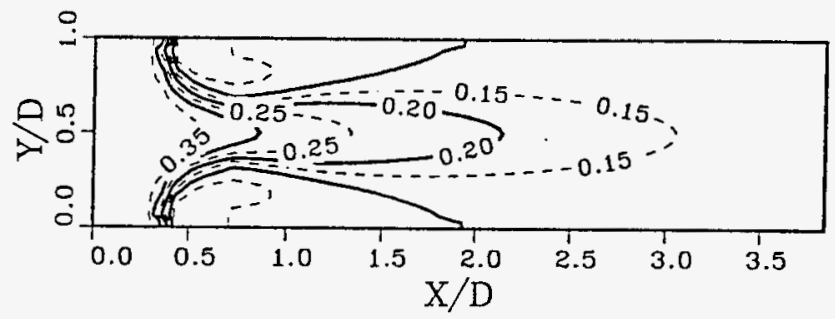

(b) Fuel

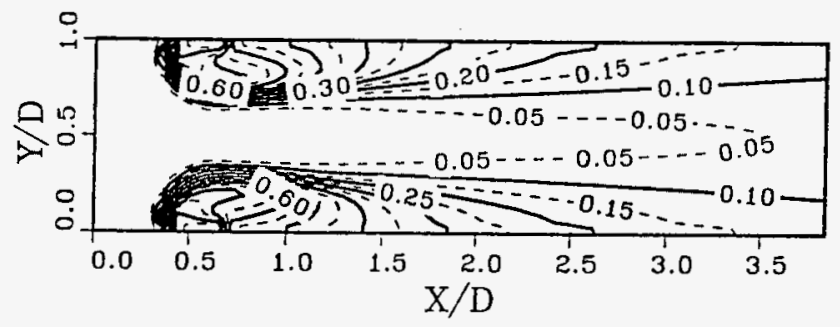

(c) Oxidizer

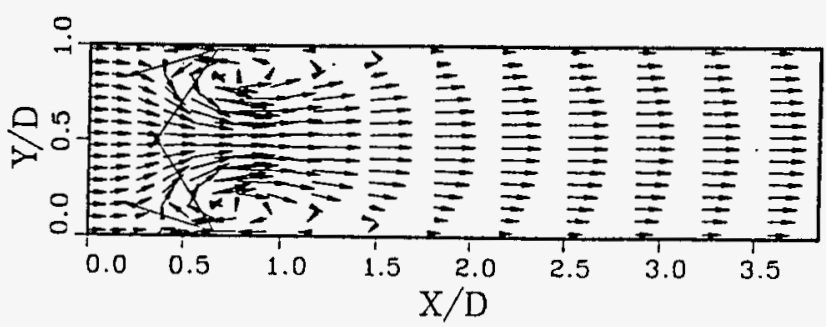

(d) Velocity

Figure 22 Sample Results of Computed Flow Fields in the Combustor 


\subsection{Numerical Stability}

In a reacting flow calculation, combustion processes generally cause severe numerical instability problems. The conventional differential reaction submodel runs into the problem of iteratively oscillating between over- and under-prediction of combustion rates in cells enclosing zones of intense combustion. In the differential model, this problem prevents convergence from dropping below a certain fixed level, depending on grid resolution and flame position within the grid.

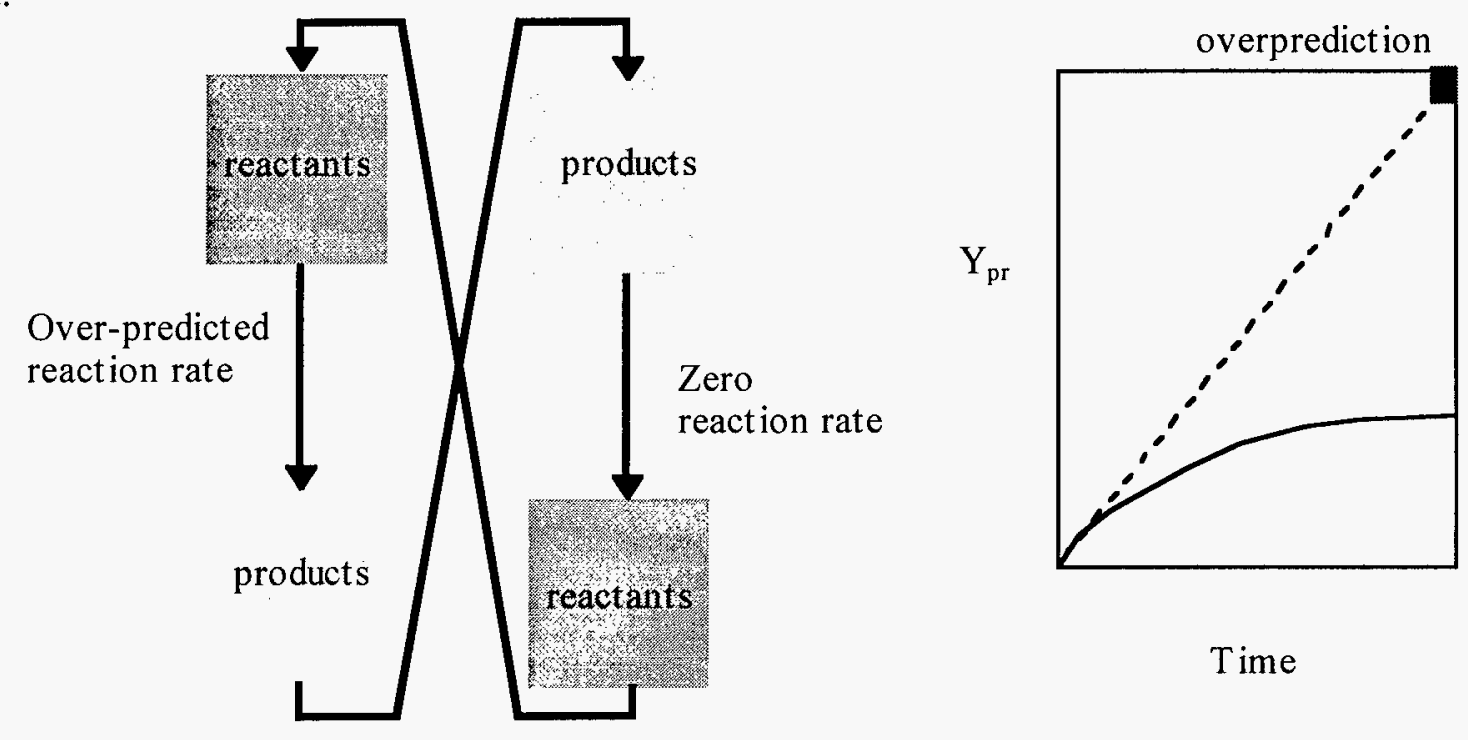

Unstable and divergent results

Figure 23 Oscillatory Numerical Instability due to Differential Approach

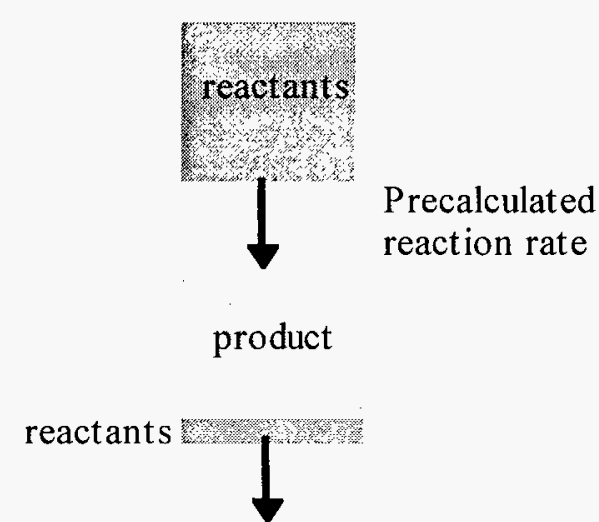

Convergent results

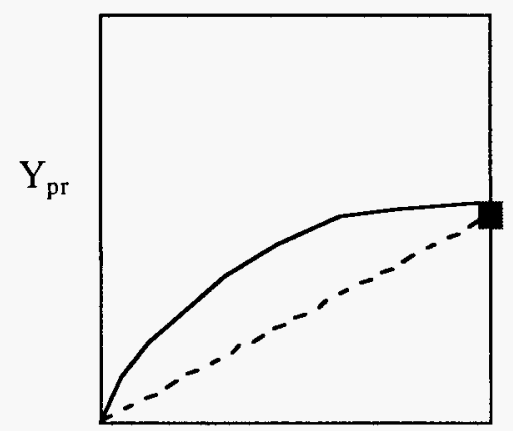

Time

Figure 24 Converged Calculation, Using the Integral Approach 
Figure 23 shows an example of oscillatory instability due to the differential reaction rate approach. In one iteration, a computational cell is filled with reactants. The differential approach overpredicts the reaction rate, therefore, reactants are all converted to products, leaving the cell with products only. In the next iteration, the cell is filled with products, so that the reaction rate is zero. Fresh reactants flow into the cell. In the third iteration, the flow conditions repeat those of the first iteration. Thus, calculations become oscillatory, and the results are divergent. On the other hand, Fig. 24 shows a different integral approach that can achieve numerical convergence.

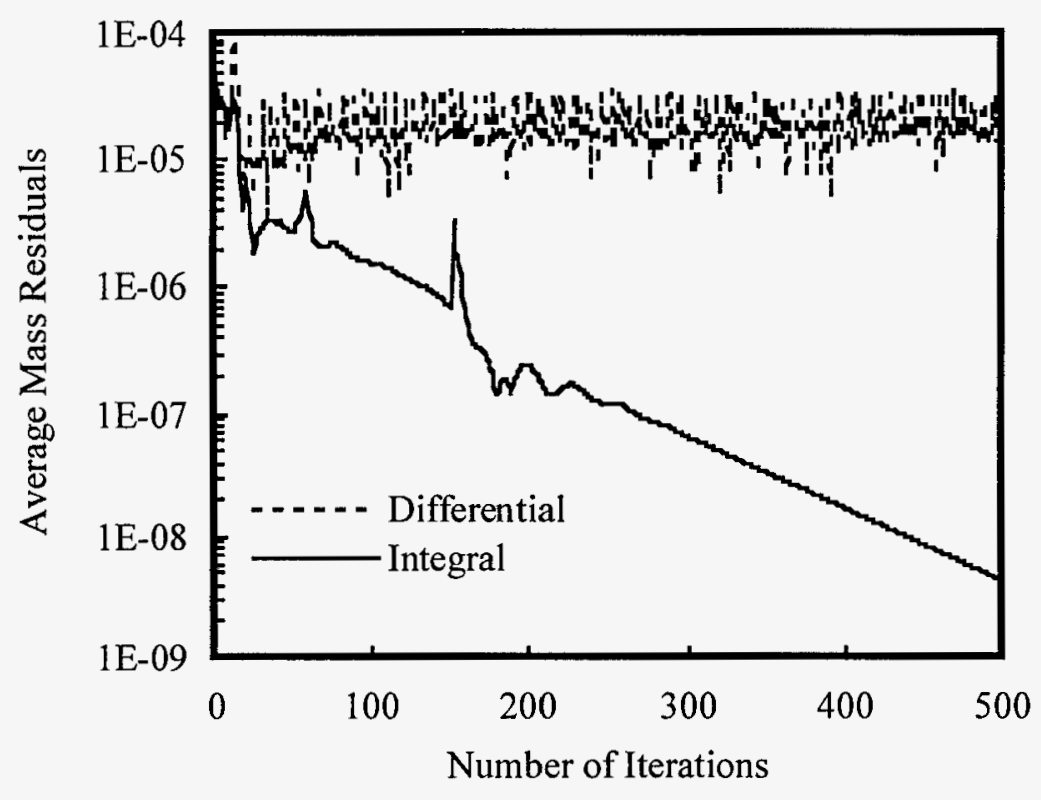

Figure 25 Numerical Convergence of Integral and Differential Reaction Rate Approaches

Figure 25 compares the convergence behavior of typical computations using the previous differential combustion model to that in the newly developed integral combustion model. It shows the clear superiority of the integral combustion model in allowing convergence based on cell mass residual to drop below the limit encountered with the differential model and head on down toward the precision limit of the machine. In these cases, computation was performed on a CRAY supercomputer in single precision, which provides between 14 and 15 significant figures. Each iteration takes approximately 0.66 second of computing time, and a typical convergent case needs about 400 seconds of supercomputing time to reduce the average mass residual to less than $10^{-9}$. 


\subsection{Code Validation}

Multiphase reacting flow fields are governed by a set of many nonlinear partial differential equations. There are no general analytical solutions for all flow systems. Approximate solutions may be obtained by various numerical iteration techniques. Due to the nature of the nonlinear governing equations, an iterative calculation often diverges, and when the calculation converges it may not approach the true solution. Besides, a general CFD computer code always employs empirical constants (i.e., turbulence parameters, reaction rate constants, specific heats, etc.) in phenomenological submodels for complex physical and chemical processes. Some constants, i.e., turbulence parameters, are estimations with a high degree of uncertainty. Therefore, a CFD code has to be validated by comparing computed results with experimental data. The ICOMFLO code has been used in many studies and validated when experimental data were available.

The ICOMFLO code was used in a study of fluid mixing in the MHD second-stage combustor [10]. This work proceeded in parallel with nonreacting flow jet injection experimental studies on the Plexiglas mockup of the second-stage combustor at TRW [29,30]. Figure 26 shows exit jet mass concentration profiles for these cases, compared with TRW experimental data. The calculation results and experimental data show very good agreement.

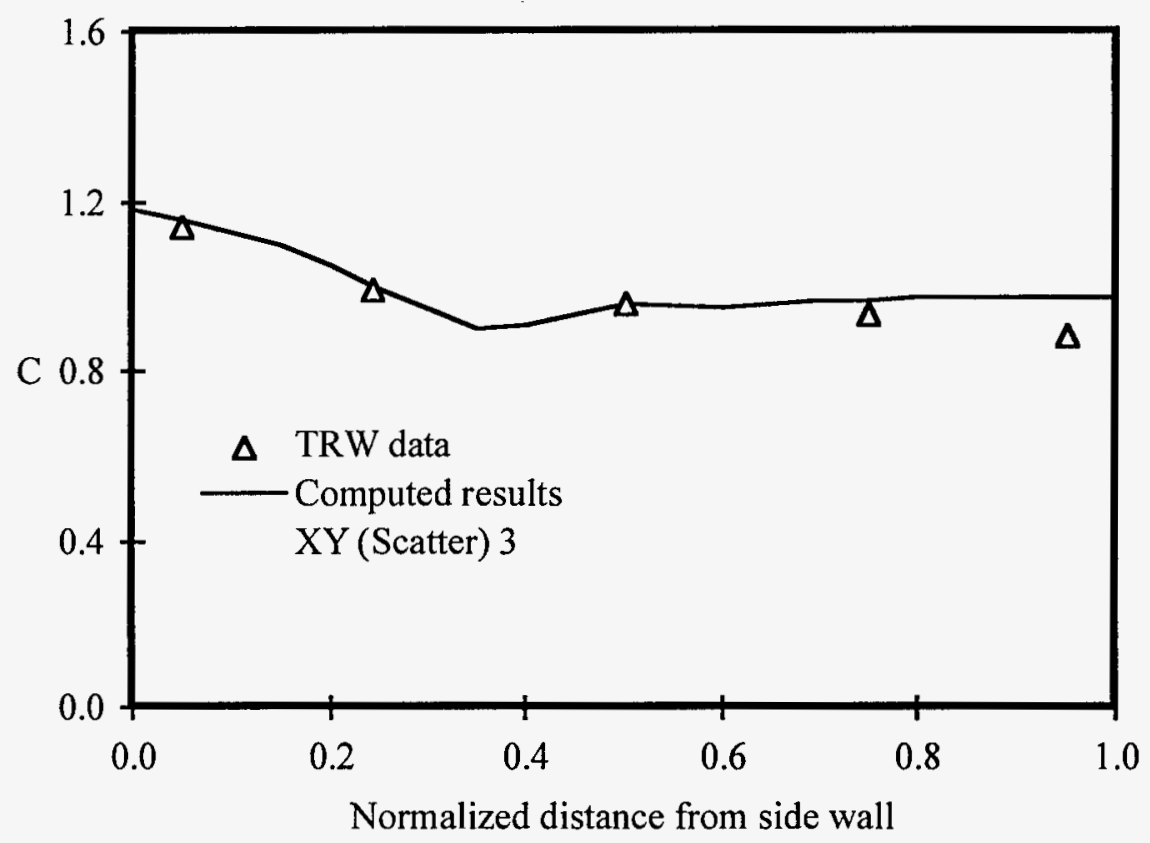

Figure 26 Comparison of Computed and Measured Results in a Fluid Mixing Study [6]

In a study of the effects of oxidizer injection on the performance of the MHD second-stage combustor [11], it was found that (1) fluid mixing is mainly responsible for combustion performance and (2) counter-flow injection with an injection angle in the range of 120 to 140 degrees, yields the best mixing and combustion performance. These were confirmed by test results 
collected at the CDIF and implemented in the final combustor design by TRW [22]. In a study of the particle flow characteristics in the MHD second-stage combustor [14], it was found that slag particles deposit on the wall just upstream of the oxidizer jet opening. This was confirmed by observation of the combustor after test at CDIF [31]. In a study of the iron oxide particle flow characteristics in the converging nozzle preceding the MHD channel [15], it predicted that the concentration of iron oxide particles in the center portion of the MHD channel due to the nozzle shape. Again, this was observed at the CDIF testing facility.

\subsection{Copyright Notification}

The ICOMFLO computer code has been copyrighted. 


\section{SUMMARY}

A copyrighted computational fluid dynamics computer code, ICOMFLO, has been developed for the simulation of multiphase reacting flows. In this report, the mathematical formulae, associated phenomenological submodels, and numerical approaches of the code are described in detail. A sample calculation is provided to illustrate the use of the code.

The ICOMFLO code computes the flow field state by solving conservation equations for gaseous species and droplets (or solid particles) of various sizes. General conservation laws, expressed by elliptic-type partial differential equations, are used in conjunction with various rate and state equations governing the mass, momentum, enthalpy, species, turbulent kinetic energy, and turbulent dissipation. Associated submodels of the code include an integral combustion, a two-parameter turbulence, a particle evaporation, and interfacial property exchange submodels. A newly developed integral combustion submodel replacing an Arrhenius-type differential reaction submodel has been implemented to improve numerical convergence and enhance numerical stability, making it possible to obtain well converged solutions to a much larger class of combustion flow problems than was feasible before. A two-parameter turbulence submodel is modified to include both gas and solid phases. An evaporation submodel treats not only droplet evaporation but also evaporation within the context of a droplet size distribution. Interfacial submodels use correlations to model interfacial momentum and heat transfer between the phases. An interfacial turbulent kinetic energy transfer submodel accounts for the dispersive effect of gas phase turbulence on droplets or particles in the condensed phase as a function of particle or droplet size.

The ICOMFLO code solves the governing equations in three major steps. First, a staggered grid system is used to define the flow field domain. The grid system defines gas velocity components on the surfaces of scalar variable control volumes, while the other flow properties are defined at the center of scalar control volumes. Then, the governing partial differential equations are integrated over each control volume of the grid system and transformed into discrete linearized algebraic equations. Finally, the algebraic equations are solved iteratively by using a modified and expanded SIMPLER algorithm. The results of the solution include gas flow properties (pressure, temperature, density, species concentration, velocity, and turbulence parameters) and particle flow properties (number density, temperature, velocity, and void fraction).

The sample calculation was set up for a multiphase reacting flow in a TRW second-stage combustor for MHD power generation. The calculation was performed using Microsoft FORTRAN Power Station software on a Pentium personal computer. The program code listing provided shows the program and control statements that were necessary to run the program on ANL's CRAY XMP supercomputer, which was the computer used for most of the parametric studies and runs for analysis during the course of the project. Since that time, ANL's CRAY computer has been decommissioned. The FORTRAN computer code for ICOMFLO was written to be easily portable to other computer systems, and it has been run on a CRAY YMP; an IBM mainframe; several VAX computers; a $25 \mathrm{MHz} 386 \mathrm{PC}$ with OS/2 operating system, using the Microsoft FORTRAN version 5.1 compiler running in protected mode; a 486DX2-66 PC running Microsoft FORTRAN Power Station software under Microsoft Windows 3.11; and finally, Pentium 90 PC computers, also using Microsoft FORTRAN Power Station. 


\section{REFERENCES}

1 Chiu, H.H., and T.M. Liu, "Group Combustion of Liquid Droplets," Combustion Science and Technology, 7:127-142, 1977.

2 Zhou, X.Q., and H.H. Chiu, "Spray Group Combustion Processes in Air Breathing Propulsion Combustors," AIAA/SAE/ASME 19th Joint Propulsion Conference, Seattle, Wash., AIAA-83-1323, 1983.

3 Jiang, T.L., and H.H. Chiu, "Advanced Modeling of Spray Combustion Process in Air Breathing Propulsion Combustors," AIAA 25th Aerospace Science Meeting, Reno, Nev., AIAA-87-0067, 1987.

4 Chang, S.L., and C.S. Wang, "Thermal Radiation and Spray Group Combustion in Diesel Engines," ASME Winter Annual Meeting, Boston, Mass., HTD-81:25-34, 1987.

5 Lottes, S.A., "Unsteady Group Combustion," Ph.D. Thesis, University of Illinois at Chicago, Chicago, Ill., 1989.

6 Chang, S.L., S.A. Lottes, and G.F. Berry, "Two-Dimensional Non-Reacting Jet-Gas Mixing in an MHD Second Stage Combustor," Proceedings of the 28th Symposium on Engineering Aspects of Magnetohydrodynamics, Chicago, Ill., pp. V.3.1-V.3.10, 1990.

7 Lottes, S.A., and S.L. Chang, "Computer Simulation of Jet Penetration and Fluid Mixing in a Channel with Cross-Stream Jets," Proceedings of the 7th International Conference on Advanced Science and Technology, Argonne, Ill., pp.188-198, 1991.

8 Lottes, S.A., and S.L. Chang, "Interactions of Turbulent Eddies and Combustion in an MHD Second Stage Combustor," Proceedings of the 26th Intersociety Energy Conversion Engineering Conference, Boston, Mass., 3:69-74, 1991.

9 Berry, G.F., S.L. Chang, S.A. Lottes, and W.A. Rimkus, "Multi-Dimensional Computer Simulation of MHD Combustor Hydrodynamics," AIAA 22nd Fluid Dynamics, Plasma Dynamics \& Lasers Conference, Honolulu, Hawaii, AIAA 91-1511, 1991.

10 Lottes, S.A., and S.L. Chang, "Simulation of Combustion Processes in an MHD Second Stage Combustor," Proceedings of the 29th Symposium on Engineering Aspects of Magnetohydrodynamics, New Orleans, La., pp. III.4.1-11, 1991.

11 Chang, S.L., S.A. Lottes, and G.F. Berry, "Computation of Two-Dimensional NonReacting Jet/Main Flow Mixing in an MHD Second Stage Combustor," Numerical Heat Transfer, Part A, 20(2):223-236, 1991.

12 Chang, S.L., S.A. Lottes, and J.X. Bouillard, "Dispersion of Seed Vapor and Gas Conductivity in an MHD Second Stage Combustor," Proceedings of the 30th Symposium on Engineering Aspects of Magnetohydrodynamics, Baltimore, Md., pp. VI.3.1-11, 1992. 
13 Lottes, S.A., and S.L. Chang, "Particle-Jet Interactions in an MHD Second Stage Combustor," Proceedings of the 30th Symposium on Engineering Aspects of Magnetohydrodynamics, Baltimore, Md., pp.VI.4.1-12, 1992.

14 Chang, S.L., and S.A. Lottes, "Simulation of a Particle-Laden Combustion Flow in an MHD Second-Stage Combustor," Proceedings of the ASME Winter Annual Meeting, HT12C, Anaheim, Calif., Nov., 1992.

15 Chang, S.L., S.A. Lottes, J.X. Bouillard, and M. Petrick, "Study of Multi-Phase Flow Characteristics in an MHD Power Train," Proceedings of the 31st Symposium on Engineering Aspects of Magnetohydrodynamics, Whitefish, Mont., pp. Vb.2.1-12, 1993.

16 Chang, S.L., and S.A. Lottes, "Integral Combustion Simulation of a Turbulent Reacting Flow in a Channel with Cross-Stream Injection," Numerical Heat Transfer, Part A, 24(1):25-43, 1993.

17 Lyczkowski, R.W., J.X. Bouillard, S.L. Chang, and S.A. Lottes, "State-of-the-Art Review of Computational Fluid Dynamics Modeling for Fluid-Solids Systems," Proceedings of the International Symposium on Parallel Computing in Multiphase Flow Systems Simulations, 1994 ASME Winter Annual Meeting, Chicago, Ill., 1994.

18 Chang, S.L., S.A. Lottes, and M. Petrick, "Simulation of Three-Dimensional Multi-Phase Flow Characteristics in the Deswirl Section of the CDIF MHD Power Train," Proceedings of the 32nd Symposium on Engineering Aspects of Magnetohydrodynamics, Pittsburgh, Penn., pp. 9.1-10, 1994.

19 Launder, B.E., and D.B. Spalding, "The Numerical Computation of Turbulent Flows," Computer Methods in Applied Mechanics and Engineering, Vol. 3, pp. 269-289, 1974.

20 Bittker, D.A., and V.J. Scullin, "General Chemical Kinetics Computer Program for Static and Flow Reactions, with Application to Combustion and Shock-tube Kinetics," National Aeronautics and Space Administration Report TN D-6586, 1972.

21 Grove, A., "Design Study of MHD Coal-Fired Combustor Second Stage," Proceedings of the 29th Symposium on Engineering Aspects of Magnetohydrodynamics, New Orleans, La., pp. III.3.1-III.3.9, June 18-20, 1991.

22 Braswell, R., T. Koyama, M. McAllister, S. Myrick, and B. Pote, "50 MWt Prototypical Combustor Performance," Proceedings of the 31st Symposium on Engineering Aspects of Magnetohydrodynamics, Whitefish, Mont., pp. II.1.1-17, 1993.

23 Pitz, W.J., and C.K. Westbrook, "Chemical Kinetics of the High Pressure Oxidation of nButane and Its Relation to Engine Knock," Combustion and Flame, Vol. 63, pp. 113-133, 1986.

24 Ward, P., N. Collings, and N. Hay, "A Comparison of Simple Models of Turbulent Droplet Diffusion Suitable for Use in Computations of Spray Flames," ASME paper no. 82WA/HT-2, 1982. 
25 Williams, F.A., "Combustion Theory," Benjamin/Cummings Pub. Co., Inc., 1985.

26 Aggarwal, S.K., A.Y. Tong, and W.A. Sirignano, "A Comparison of Vaporization Models in Spray Calculations," AIAA Journal 22(10):1448-1457, 1984.

27 Patankar, S.P., "Numerical Heat Transfer and Fluid Flow," McGraw-Hill, 1980.

28 "Microsoft FORTRAN PowerStation: User's Guide," Professional Development System, Version 1.0, Microsoft Corporation, 1992.

29 Wilson, W.W., D.V. Srikantaiah, and A. Grove, "Cold Flow Mixing Study of an MHD Combustor Model Using Laser Velocimeter and Concentration Measurements," Proceedings of the 28th Symposium on Engineering Aspects of Magnetohydrodynamics, Chicago, Ill., pp. V.2.1-V.2.8, 1990.

30 Grove, A., "Cold Flow Mixing Study of 50 MWt MHD Coal-Fired Combustor," Proceedings of the 27th Symposium on Engineering Aspects of Magnetohydrodynamics, Reno, Nev., pp. 7.1.1-7.1.9, 1989.

31 Albright, J., M. Bauer, R. Braswell, A. Grove, T. Koyama, S. Myrick, B. Pote, and H. Thom, "Demonstrated Performance of the TRW Pressurized Slagging Coal Combustor during the MHD POC Program," Proceedings of the 32nd Symposium on Engineering Aspects of Magnetohydrodynamics, Pittsburgh, Penn., 1994. 


\section{APPENDICES}

\section{Description of ICOMFLO Variables}

\begin{tabular}{|c|c|}
\hline $\begin{array}{l}\text { Variable } \\
A C D D\end{array}$ & $\begin{array}{c}\text { Description } \\
\text { coefricients in psi function for drop turb. diffusivity }\end{array}$ \\
\hline $\begin{array}{l}\text { ACDD } \\
\text { AVEB }\end{array}$ & $\begin{array}{l}\text { coeticients in psi function for drop turb. diffusivity } \\
\text { average mass residual }\end{array}$ \\
\hline $\begin{array}{l}\text { AVEB } \\
\text { BETAJN }\end{array}$ & north jet angle \\
\hline BETAJS & south jet angle \\
\hline BGCON & cut-off mass residual for gas flow \\
\hline BS & source term (constant) \\
\hline BSCON & cut-off mass residual for particle flow \\
\hline $\mathrm{Cl}$ & turbulent constant \\
\hline $\mathrm{C} 2$ & turbulent constant \\
\hline CG1 & turbulent constant \\
\hline CG2 & turbulent constant \\
\hline CTEA & turbulent constant \\
\hline $\mathrm{CR}$ & turbulent constant \\
\hline CTDA & turbulent constant \\
\hline $\mathrm{CM}$ & turbulent constant \\
\hline $\mathrm{CL}$ & particle specific heat \\
\hline $\mathrm{CPO}$ & reference specific heat \\
\hline $\mathrm{CPF}$ & specific heat of fuel \\
\hline CPIR & specific heat of inert gas \\
\hline CPMIX & specific heat of mixture \\
\hline CPOX & specific heat of oxidizer \\
\hline CPPRO & specific heat of product \\
\hline CPSV & specific heat of seed vapor \\
\hline CRATE & reaction rate \\
\hline DDENDS & particle density \\
\hline DELRT & extent of reaction function (time) \\
\hline DELR & extent of reaction \\
\hline DMFF & reference mass flow rate \\
\hline DN & particle number density \\
\hline DND0 & referenced particle number density \\
\hline DNST & gas density \\
\hline DR & $y$-cell height \\
\hline DT & particle temperature \\
\hline DU & particle axial velocity \\
\hline DV & particle transverse velocity \\
\hline DX & $x$-cell length \\
\hline ELH & latent heat \\
\hline EVP & evaporation rate \\
\hline GDFO & gas phase mass diffusion coefficient \\
\hline GDIFF & diffusivity \\
\hline GF & general variables (index) \\
\hline GFIN & inlet mass flow rate \\
\hline GJNN & north jet mass flow rate \\
\hline GJINS & south jet mass flow rate \\
\hline GLO & gas priase thermal conductivity \\
\hline G.MILO & gas phase molecular viscosity \\
\hline GRA & gravitational acceleration \\
\hline
\end{tabular}




\begin{tabular}{|c|c|}
\hline Variable & Description \\
\hline HRATE & heat release rate \\
\hline HRT & heat of reaction constants (time) \\
\hline HRD & heat of reaction constants (extent of reaction) \\
\hline IBAKUP & index for generating backup file \\
\hline IBCELL & block-cell index \\
\hline IDROP & index for particle parameter SIGMA \\
\hline IEPS & GF index for turbulent dissipation rate \\
\hline $\mathrm{IH}$ & GF index for enthalpy \\
\hline IJE & east end of jet opening \\
\hline IJW & west end of jet opening \\
\hline IK & GF index for turbulent kinetic energy \\
\hline एPHI & GF index for richness \\
\hline IRSTYP & restart type index \\
\hline TYF & GF index for fuel \\
\hline TYIR & GF index for inert gas \\
\hline IYOX & GF index for oxidizer \\
\hline IYPR & GF index for product gas \\
\hline IYSV & GF index for seed vapor \\
\hline LEND & ending number for the GF calculation \\
\hline LSTAR & starting number for the GF calculation \\
\hline MAXGI & maximal number of iterations for gas phase calculation \\
\hline MAXSI & maximal number of iterations for condensed phase calculation \\
\hline $\mathrm{MP}$ & total $x$-grid points \\
\hline $\mathrm{MZ}$ & number of $x$-cells \\
\hline $\mathrm{ND}$ & number of particle sizes \\
\hline NP & total $y$-grid points \\
\hline NPHAS & number of phases \\
\hline NR & number of kinetics constants \\
\hline NT,NS: & parameters in inlet droplet size distribution \\
\hline NZ & number of $x$-cells \\
\hline OXWJN & north jet oxidizer concentration \\
\hline OXWJS & south jet oxidizer concentration \\
\hline $\mathrm{P}$ & pressure \\
\hline $\mathrm{PG}$ & reference pressure \\
\hline PSI & particle turbulent parameters \\
\hline $\mathrm{R}$ & $y$-grid \\
\hline $\mathrm{RD}$ & particle diameter \\
\hline REACT & index for reaction flow calculation \\
\hline $\mathrm{RF}$ & relaxation factor \\
\hline RLE & chamber height \\
\hline RLMO & mean particle size \\
\hline RTMM & kinetic reacting time scale \\
\hline TIMED & kinetic reacting time scale \\
\hline RU & universal gas constant \\
\hline SIGMA & ratio of property diffusivity to efective viscosity \\
\hline SFP & source term (linear) \\
\hline SMX & maximal mass residual \\
\hline STOICH & stoichiometric ratio of oxidizer to fuel \\
\hline $\mathrm{TB}$ & boil point temperature \\
\hline
\end{tabular}




\begin{tabular}{ll}
\multicolumn{1}{c}{ Variable } & \\
THETA & gas void fraction \\
TINF & reference temperature \\
TMU & turbulent diffusivity \\
U & axial velocity \\
V & transverse velocity \\
VJMAGN & north jet velocity \\
VJMAGS & south jet velocity \\
WF & molecular weight of fuel \\
WIDTH & chamber width perpendicular to simulated plane \\
WIR & molecular weight of inert gas \\
WJMF & total wall jet mass flow rate \\
WOX & molecular weight of oxidizer \\
WPR & molecular weight of product \\
WSV & molecular weight of seed vapor \\
WT & molecular weight of inlet gas mixture \\
WTJN & molecular weight of north jet gas mixture \\
WTJS & molecular weight of south jet gas mixture \\
X & x-grid \\
XLE & chamber length \\
YFWJN & north jet fuel concentration \\
YFWJS & south jet fuel concentration \\
YIRWJN & north jet inert gas concentration \\
YIRWJS & south jet iner gas concentration \\
YPRWJN & north jet product concentration \\
YPRWJS & south jet product concentration \\
YSVWJN & north jet seed vapor concentration \\
YSVWJS & south jet seed vapor concentration \\
NTIMES & number of iterations for solving each governing equation \\
REYG & Reynolds number \\
REYD & Reynolds number \\
SCN & Schmidt number \\
DNUL0 & Nusselt number \\
DNUC0 & Nusselt number \\
GCN & group combustion number \\
GVN & particle heating coefficient \\
ZO & particle drag correlation factor \\
GDN & group particle drag numer \\
EUN & Euler number \\
ECN & Eckert number \\
&
\end{tabular}




\section{Kinetic Constant File for Sample Calculation}

\begin{tabular}{|c|c|c|c|c|c|}
\hline 0 & & & 2.0 & & \\
\hline .0500 & .2495 & 10.0300 & .0057 & .0007 & 2214 \\
\hline .1000 & .3259 & 17.2276 & .0114 & .0013 & .4428 \\
\hline .1500 & .3631 & 22.6513 & .0171 & .0020 & .6642 \\
\hline 2000 & .3822 & 26.8449 & .0228 & .0025 & .8350 \\
\hline .2500 & .3926 & 30.0360 & .0285 & .0030 & 1.0038 \\
\hline .3000 & .4000 & 33.2540 & .0342 & .0036 & 1.1627 \\
\hline .3500 & .4062 & 35.8480 & .0399 & .0042 & 1.3216 \\
\hline .4000 & .4118 & 38.0182 & .0456 & .0047 & 1.4805 \\
\hline .4500 & .4173 & 39.8334 & .0513 & .0053 & 1.6380 \\
\hline .5000 & .4228 & 41.3807 & .0570 & .0059 & 1.7943 \\
\hline .5500 & .4282 & 42.7412 & .0627 & .0065 & 1.9520 \\
\hline .6000 & .4335 & 43.8963 & .0684 & .0071 & 2.1108 \\
\hline .6500 & .4385 & 44.9116 & .0741 & .0078 & 2.2721 \\
\hline .7000 & .4436 & 45.8161 & .0798 & .0085 & 2.4344 \\
\hline .7500 & .4485 & 46.6157 & .0855 & .0092 & 2.5979 \\
\hline .8000 & .4532 & 47.3288 & .0912 & .0103 & 2.7636 \\
\hline .8500 & .4577 & 47.9674 & .0969 & .0112 & 2.9312 \\
\hline .9000 & .4622 & 48.5476 & .1026 & .0119 & 3.1029 \\
\hline .9500 & .4655 & 49.0689 & .1083 & .0129 & 3.2779 \\
\hline 1.0000 & .4706 & 49.5504 & .1140 & .0139 & 3.4536 \\
\hline 1.0500 & .4745 & 49.9746 & .1197 & .0149 & 3.6383 \\
\hline 1.1000 & .4783 & 50.3732 & .1254 & .0159 & 3.8239 \\
\hline 1.1500 & .4820 & 50.7421 & .1311 & .0168 & 4.0175 \\
\hline 1.2000 & .4856 & 51.0775 & .1368 & .0177 & 4.2186 \\
\hline 1.2500 & .4890 & 51.3869 & .1425 & .0186 & 4.4227 \\
\hline 1.3000 & .4923 & 51.6787 & .1482 & .0200 & 4.6305 \\
\hline 1.3500 & .4955 & 51.9410 & .1539 & .0213 & 4.8473 \\
\hline 1.4000 & .4986 & 52.1905 & .1596 & .0223 & 5.0751 \\
\hline 1.4500 & .5016 & 52.4236 & .1653 & .0235 & 5.3081 \\
\hline 1.5000 & .5045 & 52.6413 & .1710 & .0248 & 5.5498 \\
\hline 1.5500 & .5072 & 52.8448 & .1767 & .0261 & 5.7987 \\
\hline 1.6000 & .5098 & 53.0339 & .1824 & .0276 & 6.0624 \\
\hline 1.6500 & .5124 & 53.2143 & .1881 & .0292 & 6.3349 \\
\hline 1.7000 & .5148 & 53.3803 & .1938 & .0310 & 6.6112 \\
\hline 1.7500 & .5172 & 53.5365 & . 1995 & .0322 & 6.9078 \\
\hline 1.8000 & .5195 & 53.6880 & .2052 & .0337 & 7.2135 \\
\hline 1.8500 & .5216 & 53.8248 & .2109 & .0354 & 7.5321 \\
\hline 1.9000 & .5237 & 53.9561 & .2166 & .0375 & 7.8613 \\
\hline 1.9500 & .5257 & 54.0810 & .2223 & .0399 & 8.1973 \\
\hline 2.0000 & .5278 & 54.1965 & .2280 & .0419 & 8.5597 \\
\hline 2.0500 & .5296 & 54.3060 & .2337 & .0438 & 8.9309 \\
\hline 2.1000 & .5314 & 54.4102 & .2394 & .0456 & 9.3115 \\
\hline 2.1500 & .5331 & 54.5082 & .2451 & .0480 & 9.7139 \\
\hline 2.2000 & .5347 & 54.5994 & .2508 & .0506 & 10.1279 \\
\hline 2.2500 & .5364 & 54.6872 & .2565 & .0535 & 10.5572 \\
\hline 2.3000 & .5379 & 54.7709 & .2622 & .0561 & 11.0080 \\
\hline 2.3500 & .5393 & 54.8478 & .2679 & .0588 & 11.4699 \\
\hline 2.4000 & .5407 & 54.9217 & .2736 & .0617 & 11.9459 \\
\hline 2.4500 & .5421 & 54.9909 & .2793 & .0654 & 12.4440 \\
\hline 2.5000 & .5434 & 55.0543 & .2850 & .0690 & 12.9569 \\
\hline 2.5500 & .5446 & 55.1150 & .2907 & .0724 & 13.4841 \\
\hline 2.6000 & .5458 & 55.1708 & .2964 & .0759 & 14.0304 \\
\hline 2.6500 & .5469 & 55.2253 & .3021 & .0799 & 14.5082 \\
\hline 2.7000 & .5480 & 55.2765 & .3078 & .0844 & 15.1961 \\
\hline 2.7500 & .5490 & 55.3242 & .3135 & .0893 & 15.8131 \\
\hline 2.8000 & .5501 & 55.3687 & .3192 & .0943 & 16.4440 \\
\hline 2.8500 & .5510 & 55.4106 & .3249 & .0990 & 17.1142 \\
\hline 2.9000 & .5520 & 55.4511 & .3306 & .1050 & 17.8054 \\
\hline 2.9500 & .5528 & 55.4872 & .3363 & .1107 & 18.5362 \\
\hline 3.0000 & .5535 & 55.5220 & .3420 & .1174 & 19.3107 \\
\hline 3.0500 & .5544 & 55.5539 & .3477 & .1247 & 20.1319 \\
\hline 3.1000 & 5552 & 55.5849 & .3534 & .1332 & 21.0119 \\
\hline 3.1500 & .5559 & 55.6125 & .3591 & .1424 & 21.9534 \\
\hline
\end{tabular}


79

$\begin{array}{lll}3.2000 & .5565 & 55.6389 \\ 3.2500 & .5572 & 55.6635 \\ 3.3000 & .5578 & 55.6867 \\ 3.3500 & .5584 & 55.7086 \\ 3.4000 & .5590 & 55.7279 \\ 3.4500 & .5596 & 55.7472 \\ 3.5000 & .5601 & 55.7630 \\ 3.5500 & .5605 & 55.7786 \\ 3.6000 & .5610 & 55.7933 \\ 3.6500 & .5615 & 55.8069 \\ 3.7000 & .5619 & 55.8205 \\ 3.7500 & .5623 & 55.8317 \\ 3.8000 & .5626 & 55.8424 \\ 3.8500 & .5630 & 55.8530 \\ 3.9000 & .5634 & 55.8613 \\ 3.9500 & .5637 & 55.8696 \\ 4.0000 & .5641 & 55.8778 \\ 4.0500 & .5644 & 55.8837 \\ 4.1000 & .5646 & 55.8895 \\ 4.1500 & .5649 & 55.8953 \\ 4.2000 & .5652 & 55.9001 \\ 4.2500 & .5654 & 55.9046 \\ 4.3000 & .5657 & 55.9091 \\ 4.3500 & .5659 & 55.9134 \\ 4.4000 & .5661 & 55.9162 \\ 4.4500 & .5663 & 55.9189 \\ 4.5000 & .5665 & 55.9217 \\ 4.5500 & .5666 & 55.9243 \\ 4.6000 & .5668 & 55.9258 \\ 4.6500 & .5670 & 55.9274 \\ 4.7000 & .5672 & 55.9290 \\ 4.7500 & .5673 & 55.9305 \\ 4.8000 & .5675 & 55.9313 \\ 4.8500 & .5676 & 55.9317 \\ 4.9000 & .5677 & 55.9320 \\ 4.9500 & .5678 & 55.9324 \\ 7.5160 & .5699 & 55.9328\end{array}$

$\begin{array}{lrl}.3648 & .1532 & 22.9752 \\ .3705 & .1656 & 24.1042 \\ .3762 & .1809 & 25.3525 \\ .3819 & .1990 & 26.7720 \\ .3876 & .2227 & 28.4037 \\ .3933 & .2539 & 30.3078 \\ .3990 & .2924 & 32.7905 \\ .4047 & .3373 & 35.2428 \\ .4104 & .3870 & 37.4891 \\ .4161 & .4387 & 39.4453 \\ .4218 & .4913 & 41.1233 \\ .4275 & .5437 & 42.5762 \\ .4332 & .5970 & 43.8339 \\ .4389 & .6537 & 44.9803 \\ .4446 & .7106 & 45.9935 \\ .4503 & .7688 & 46.8992 \\ .4560 & .8310 & 47.7360 \\ .4617 & .8947 & 48.4912 \\ .4674 & .9612 & 49.1778 \\ .4731 & 1.0320 & 49.8221 \\ .4788 & 1.1061 & 50.4217 \\ .4845 & 1.1844 & 50.9792 \\ .4902 & 1.2675 & 51.4907 \\ .4959 & 1.3556 & 51.9702 \\ .5016 & 1.4502 & 52.4245 \\ .5073 & 1.5516 & 52.8510 \\ .5130 & 1.6622 & 53.2581 \\ .5187 & 1.7830 & 53.6364 \\ .5244 & 1.9173 & 54.0004 \\ .5301 & 2.0634 & 54.3350 \\ .5358 & 2.2322 & 54.6574 \\ .5415 & 2.4292 & 54.9621 \\ .5472 & 2.6638 & 55.2394 \\ .5529 & 2.9572 & 55.4922 \\ .5586 & 3.3670 & 55.7151 \\ .5643 & 4.0374 & 55.8822 \\ .5699 & 7.5160 & 55.9328\end{array}$




\section{Common Biock File for Sample Calculation}

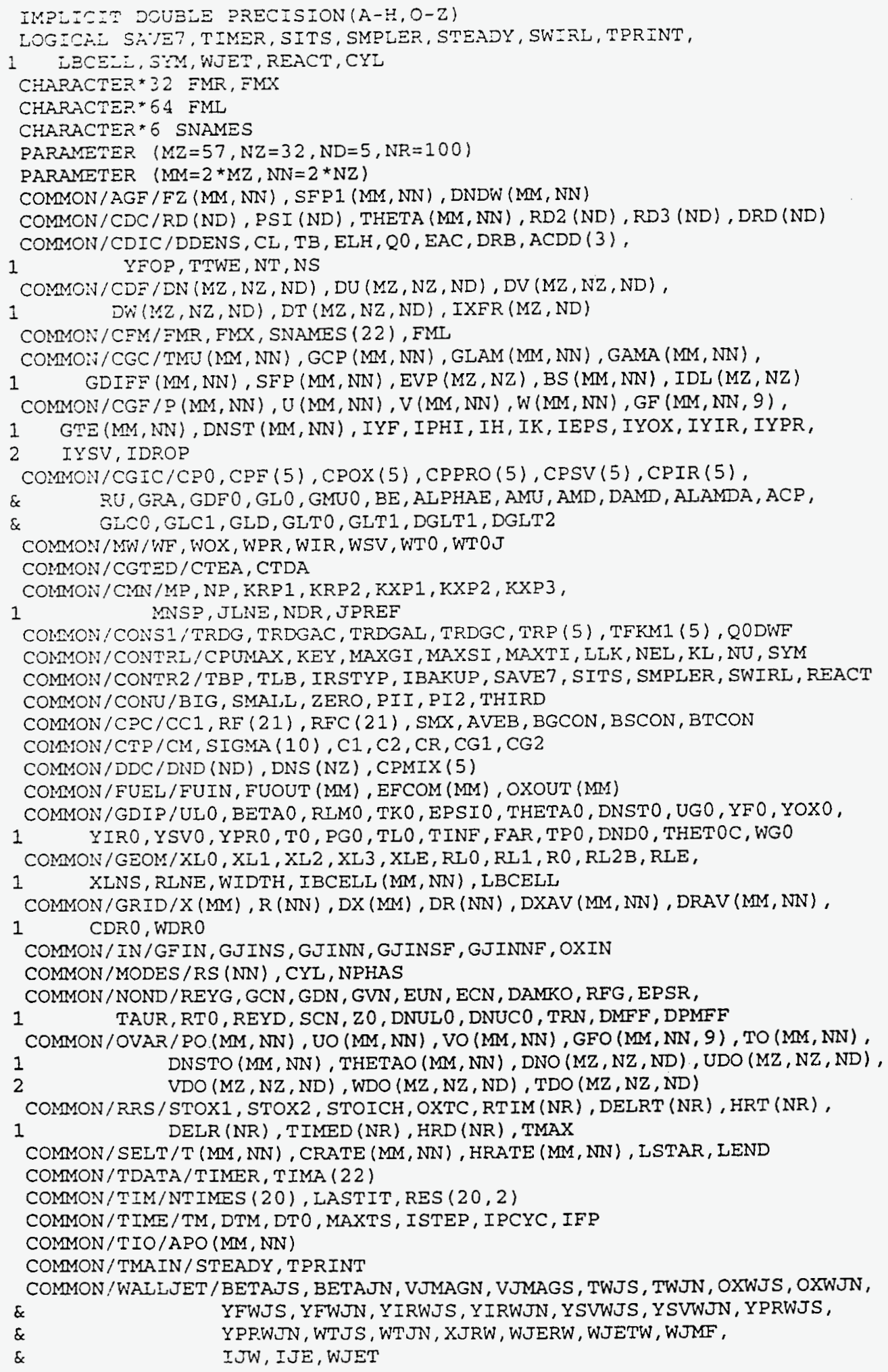



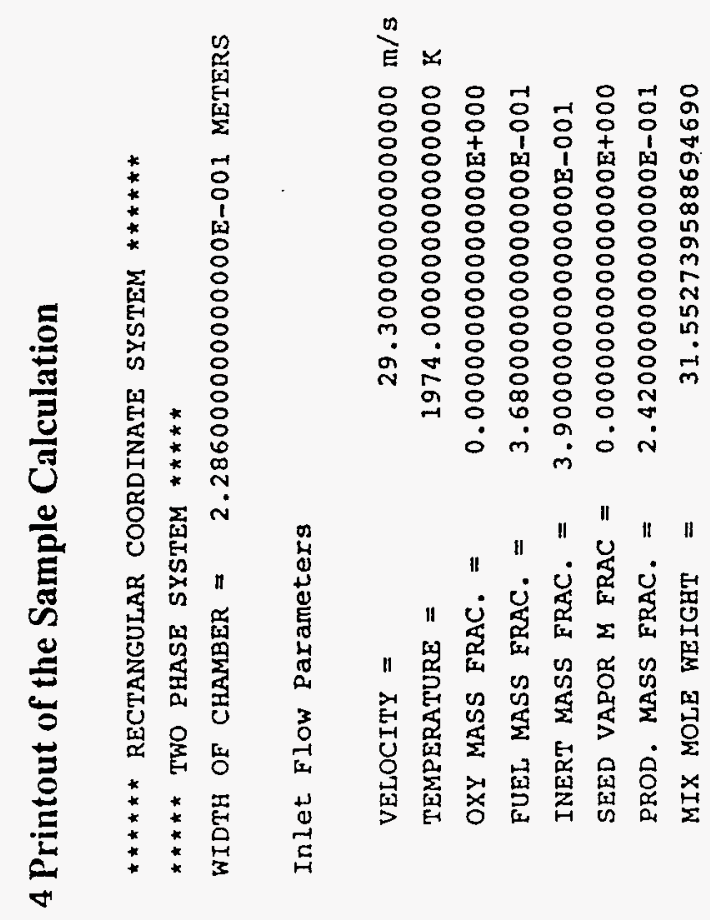

哭哭

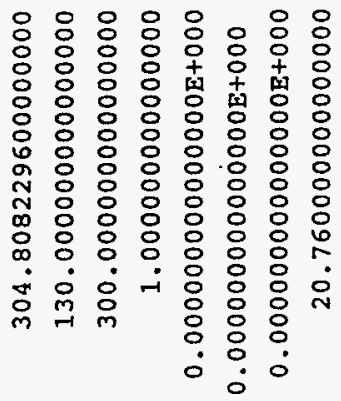

号

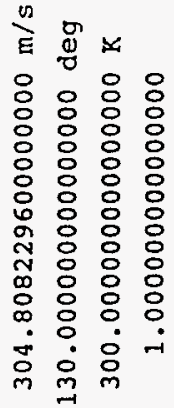

急 
EUEL MASS FRAC. $=0.000000000000000 \mathrm{E}+000$

INERT MASS FRAC. $=0.000000000000000 \mathrm{E}+000$

PROD. MASS FRAC. $=0.000000000000000 \mathrm{E}+000$

MIX MOLE WEIGHT $=20.760000000000000$

Reference density $=\quad 1.110339275165852 \mathrm{~kg} /$ cubic meters

Normalized boiling temperature $=8.080040526849037 \mathrm{E}-001$

GRID ASSIGNMENT

\begin{tabular}{|c|c|c|c|c|c|c|c|c|c|c|c|}
\hline$x<$ & 1) $=$ & .0000 & DX & $1)=$ & .0000 & $x($ & $58)=$ & .9986 & $\mathrm{DX}($ & $58)=$ & .0719 \\
\hline$x($ & $2)=$ & .0000 & $\mathrm{DX}($ & $2)=$ & .0000 & $x($ & $59)=$ & 1.0346 & $\mathrm{DX}($ & $59)=$ & .0759 \\
\hline$x($ & 3) $=$ & .0000 & $\mathrm{DX}($ & $3)=$ & .0231 & $x($ & $60)=$ & 1.0745 & $\mathrm{DX} 6$ & $60)=$ & .0799 \\
\hline $\mathrm{xi}$ & $4)=$ & .0231 & $\mathrm{DX}($ & $4)=$ & .0462 & $x($ & $61)=$ & 1.1145 & $\mathrm{DX}($ & $61)=$ & .0839 \\
\hline$x<$ & 5) = & .0462 & $\mathrm{DX}($ & $5)=$ & .0462 & $x($ & $62)=$ & 1.1584 & $\mathrm{DX} 6$ & $62)=$ & .0879 \\
\hline$x !$ & $6)=$ & .0693 & $\mathrm{DX}($ & $6)=$ & .0462 & $x($ & $63)=$ & 1.2024 & DXI & $63)=$ & .0919 \\
\hline$x($ & 7) $=$ & .0924 & $\mathrm{DX}($ & $7)=$ & .0462 & $x($ & $64)=$ & 1.2503 & $\mathrm{DX}($ & $64)=$ & .0959 \\
\hline$x($ & $8)=$ & .1156 & $\mathrm{DX}($ & $8)=$ & .0462 & $x($ & $65)=$ & 1.2983 & $D \times($ & $65)=$ & .0999 \\
\hline$x($ & $9)=$ & .1387 & $\mathrm{DX}($ & 9)= & .0462 & $x i$ & $66)=$ & 1.3502 & $\mathrm{DX}($ & $66)=$ & .1039 \\
\hline$x($ & $10)=$ & .1618 & $\mathrm{DX} 6$ & $10)=$ & .0462 & $x($ & $67)=$ & 1.4021 & $D \times($ & $67)=$ & .1079 \\
\hline$x i$ & $11\rangle=$ & .1849 & $\mathrm{DX}($ & $11)=$ & .0462 & $x($ & $68)=$ & 1.4581 & $\mathrm{DX}($ & $68)=$ & .1119 \\
\hline$x($ & $12)=$ & .2080 & $\mathrm{DX}($ & $12)=$ & .0462 & $x($ & $69)=$ & 1.5140 & $D X($ & $69)=$ & .1149 \\
\hline$x($ & $13)=$ & .2311 & $\mathrm{DX}($ & $13)=$ & .0462 & $x($ & $70)=$ & 1.5730 & $D \times($ & $70)=$ & .1179 \\
\hline$x($ & $14)=$ & .2542 & DXI & $14)=$ & .0462 & $x($ & $71)=$ & 1.6319 & $D \times($ & $71)=$ & .1179 \\
\hline$x 1$ & $15)=$ & .2773 & DX & $15)=$ & .0462 & $x($ & $72)=$ & 1.6909 & $D X($ & $72)=$ & .1179 \\
\hline$x($ & $16)=$ & .3004 & $\mathrm{DX}($ & $16)=$ & .0462 & $x($ & $73)=$ & 1.7498 & $\mathrm{DX}($ & $73)=$ & .1179 \\
\hline$x($ & $17)=$ & .3236 & DX( & 17)= & .0462 & $x($ & $74)=$ & 1.8088 & DXI & $74)=$ & .1179 \\
\hline$x 1$ & $18)=$ & .3467 & $\mathrm{DX}($ & $18)=$ & .0462 & $x($ & $75)=$ & 1.8677 & DXI & $75)=$ & .1179 \\
\hline$x($ & $19)=$ & .3698 & $\mathrm{DX}($ & 19) = & .0462 & $x($ & $76)=$ & 1.9267 & DXI & $76)=$ & .1179 \\
\hline$x($ & $20)=$ & .3929 & DXI & $20)=$ & .0462 & $x($ & $77)=$ & 1.9856 & $\mathrm{DX}($ & $77)=$ & .1179 \\
\hline$x($ & $21)=$ & .4160 & DXI & $21)=$ & .0462 & $x($ & $78)=$ & 2.0446 & $\mathrm{DX}($ & $78)=$ & .1179 \\
\hline$x<$ & $221=$ & .4391 & DXI & $22)=$ & .0462 & $x i$ & $79)=$ & 2.1035 & $\mathrm{DXC}$ & $79)=$ & .1179 \\
\hline$x($ & $23)=$ & .4622 & $D \times($ & $23)=$ & .0462 & $x($ & $80)=$ & 2.1625 & DX ( & $80)=$ & .1179 \\
\hline$x($ & $24)=$ & .4853 & $D \times($ & $24)=$ & .0462 & $x($ & $81)=$ & 2.2214 & $D X($ & $81)=$ & .1179 \\
\hline$x($ & $25)=$ & .5084 & $\mathrm{DX}($ & $25)=$ & .0462 & $x($ & $82)=$ & 2.2804 & DX & $82)=$ & .1179 \\
\hline$x($ & $26)=$ & .5315 & DXI & $26)=$ & 462 & $x($ & $y=$ & 3393 & $D \times 1$ & 3) & 1179 \\
\hline
\end{tabular}




\begin{tabular}{|c|c|c|c|c|c|c|c|c|c|c|c|c|c|c|c|}
\hline$x($ & $27)=$ & .5547 & & $\mathrm{DX}($ & $27)=$ & & 0462 & & $x(84)=$ & 2.3983 & $\operatorname{DX}(84)=$ & .1179 & & & \\
\hline$x($ & $28)=$ & .5778 & & DXI & $28)=$ & & 0462 & & $x(85)=$ & 2.4573 & $D X(85)=$ & .1179 & & & \\
\hline$x($ & $29\rangle=$ & .6009 & & $\mathrm{DX}($ & 29) $=$ & & 0462 & & $x(86)=$ & 2.5162 & $\operatorname{DX}(86)=$ & .1179 & & & \\
\hline$x i$ & $30)=$ & .6240 & & $\mathrm{DX}($ & $30)=$ & & 0462 & & $x(87)=$ & 2.5752 & $\operatorname{Dx}(87)=$ & .1179 & & & \\
\hline$x($ & $31)=$ & .6471 & & $\mathrm{DX}($ & $31)=$ & & 0291 & & $x(88)=$ & 2.6341 & $\operatorname{DX}(88)=$ & .1179 & & & \\
\hline$x($ & $32)=$ & .6531 & & $\mathrm{DX}($ & $32)=$ & & 0120 & & $x(89)=$ & 2.6931 & $\operatorname{DX}(89)=$ & .1179 & & & \\
\hline$x i$ & $33)=$ & .6591 & & $\mathrm{DX}($ & $33)=$ & & 0067 & & $x(90)=$ & 2.7520 & $\operatorname{DX}(90)=$ & .1179 & & & \\
\hline$x($ & $34)=$ & .6598 & & DXI & $34)=$ & & 0013 & & $x(91)=$ & 2.8110 & $D x(91)=$ & .1179 & & & \\
\hline$x($ & $35)=$ & .6604 & & $\mathrm{DX}($ & $35)=$ & & 0013 & & $x(92)=$ & 2.8699 & $D X(92)=$ & .1179 & & & \\
\hline$x i$ & $36)=$ & .6610 & & $\mathrm{DX}($ & $36)=$ & & 0013 & & $x(93)=$ & 2.9289 & $D X(93)=$ & .1179 & & & \\
\hline$x<$ & $37)=$ & .6617 & & $\mathrm{DX}($ & $37)=$ & & 0013 & & $x(94)=$ & 2.9878 & $\operatorname{DX}(94)=$ & .1179 & & & \\
\hline$x($ & $38\}=$ & .6623 & & $\mathrm{DX}($ & $38)=$ & & 0013 & & $x(95)=$ & 3.0468 & $D X(95)=$ & .1179 & & & \\
\hline$x i$ & $39)=$ & .6630 & & $\mathrm{DX}($ & $39)=$ & & 0067 & & $x(96)=$ & 3.1057 & $\operatorname{DX}(96)=$ & .1179 & & & \\
\hline$x($ & $40)=$ & .6690 & & $\mathrm{DX}($ & $401=$ & & 0120 & & $x(97)=$ & 3.1647 & $D \times(97)=$ & .1179 & & & \\
\hline$x($ & $41)=$ & .6750 & & $\mathrm{DX}($ & $411=$ & & 0100 & & $x(98)=$ & 3.2236 & $\operatorname{Dx}(98)=$ & .1179 & & & \\
\hline$x($ & $42)=$ & .6790 & & $\mathrm{DX}($ & $421=$ & & 0080 & & $x(99)=$ & 3.2826 & $\operatorname{Dx}(99)=$ & .1179 & & & \\
\hline$x($ & $431=$ & .6830 & & $\mathrm{DX}($ & $43)=$ & & 0120 & & $x(100)=$ & 3.3416 & $\operatorname{DX}(100)=$ & .1179 & & & \\
\hline$x($ & $44)=$ & .6910 & & $\mathrm{DX}($ & $44)=$ & & 0160 & & $x(101)=$ & 3.4005 & $D X(101)=$ & .1179 & & & \\
\hline$x($ & $45)=$ & .6990 & & DX( & $45)=$ & & 0200 & & $x(102)=$ & 3.4595 & $\mathrm{DX}(102)=$ & .1179 & & & \\
\hline$x($ & $46)=$ & .7110 & & DX( & $46)=$ & & 0240 & & $x(103)=$ & 3.5184 & $\mathrm{DX}(103)=$ & .1179 & & & \\
\hline$x($ & $47)=$ & .7229 & & $\mathrm{DX}($ & 47)= & & 0280 & & $x(104)=$ & 3.5774 & $\operatorname{DX}(104)=$ & .1179 & & & \\
\hline$x<$ & $48)=$ & .7389 & & $\mathrm{D} \times($ & $48)=$ & & 0320 & & $x(105)=$ & 3.6363 & $\mathrm{DX}(105)=$ & .1179 & & & \\
\hline$x($ & $49)=$ & .7549 & & $\mathrm{DX}($ & $49)=$ & & 0360 & & $x(106)=$ & 3.6953 & $\operatorname{DX}(106)=$ & .1179 & & & \\
\hline$x($ & $50)=$ & .7749 & & $\mathrm{DX}($ & $50)=$ & & .0400 & & $x(107)=$ & 3.7542 & $D \times(107)=$ & .1179 & & & \\
\hline$x($ & $511=$ & .7949 & & $\mathrm{DX}($ & $51)=$ & & 0439 & & $x(108)=$ & 3.8132 & $\operatorname{DX}(108)=$ & .1179 & & & \\
\hline$x($ & $521=$ & .8188 & & $\mathrm{DX}($ & $521=$ & & .0479 & & $x(109)=$ & 3.8721 & $D \times(109)=$ & .1179 & & & \\
\hline$x($ & $531=$ & .8428 & & DXI & $53)=$ & & .0519 & & $x(110)=$ & 3.9311 & $\operatorname{DX}(110)=$ & .1179 & & & \\
\hline$x($ & $54)=$ & .8708 & & DXI & $54)=$ & & .0559 & & $x(111)=$ & 3.9900 & $D x(111)=$ & .1179 & & & \\
\hline$x($ & $551=$ & .8987 & & $\mathrm{DX}($ & $55)=$ & & .0599 & & $x(112)=$ & 4.0490 & $D \times(112)=$ & .1179 & & & \\
\hline$x($ & $56)=$ & .9307 & & $\mathrm{DX}($ & $56)=$ & & .0639 & & $x(113)=$ & 4.1080 & $\mathrm{DX}(113)=$ & .0590 & & & \\
\hline$x i$ & $57)=$ & .9627 & & DXC & $57)=$ & & .0679 & & $x(114)=$ & 4.1080 & $\operatorname{Dx}(114)=$ & .0000 & & & \\
\hline Ri & 1) $=$ & .0000 & DR ( & 1) $=$ & & .0000 & RS ( & $1)=$ & .0000 & $R(33)=$ & $.5000 \mathrm{DR}($ & $33)=$ & .0346 & $\mathrm{RS}(33)=$ & 1.0000 \\
\hline Ri & 2) $=$ & .0000 & $\mathrm{DR}($ & $2)=$ & & .0000 & 0 RS ( & $2)=$ & 1.0000 & $R(34)=$ & .5173 DRR & $34)=$ & .0346 & $\operatorname{RS}(34)=$ & 1.0000 \\
\hline Ri & $3)=$ & .0000 & $\mathrm{DR}($ & $3)=$ & & .0080 & DS ( & $3)=$ & 1.0000 & $R(35)=$ & .5346 DR & $35)=$ & .0346 & $\operatorname{RS}(35)=$ & 1.0000 \\
\hline $\mathrm{R}($ & $4)=$ & .0080 & DR ( & $4)=$ & & .0159 & 9 RS( & 4) $=$ & 1.0000 & $R(36)=$ & .5519 DR( & $36)=$ & .0346 & $\operatorname{RS}(36)=$ & 1.0000 \\
\hline $\mathrm{R}$ ( & $5)=$ & .0159 & DRI & 5) $=$ & & .0252 & $2 \mathrm{RS}($ & $5)=$ & 1.0000 & $R(37)=$ & .5692 DR & $37)=$ & .0346 & $\operatorname{RS}(37)=$ & 1.0000 \\
\hline
\end{tabular}




\begin{tabular}{|c|c|c|c|c|c|c|c|c|c|c|c|c|c|c|c|c|}
\hline Ri & 6) $=$ & .0332 & DR( & $6)=$ & .0346 & RS ( & $6)=$ & 1.0000 & Ri & $38)=$ & .5864 & DRI & $38)=$ & .0346 & $\operatorname{RS}(38)=$ & 1.0000 \\
\hline RI & $7)=$ & .0505 & DRI & $7)=$ & .0346 & RS ( & 7) $=$ & 1.0000 & Ri & $39)=$ & .6037 & DRl & $39)=$ & .0346 & $\operatorname{RS}(39)=$ & .0000 \\
\hline Ri & $8)=$ & .0678 & DRI & $8)=$ & .0346 & RS ( & $8)=$ & 1.0000 & $\mathrm{R} /$ & $40)=$ & .6210 & $\mathrm{DR}($ & $40)=$ & .0346 & $\operatorname{RS}(40)=$ & 1.0000 \\
\hline $\mathrm{R}$ & 9) $=$ & .0851 & DRI & $9)=$ & .0346 & RS ( & $9)=$ & 1.0000 & $\mathrm{R} /$ & $41)=$ & .6383 & $\mathrm{DR}($ & $41)=$ & .0346 & $\operatorname{RS}(41)=$ & 1.0000 \\
\hline$R($ & $10)=$ & .1023 & DRI & $10)=$ & .0346 & RS ( & $10)=$ & 1.0000 & $R($ & $42)=$ & .6556 & $D R($ & $42)=$ & .0346 & $\operatorname{RS}(42)=$ & 1.0000 \\
\hline R( & $11)=$ & .1196 & DRI & $11)=$ & .0346 & RS ( & $11)=$ & 1.0000 & $\mathrm{R}($ & $43)=$ & .6729 & DR( & $43)=$ & .0346 & $\operatorname{RS}(43)=$ & 1.0000 \\
\hline Ri & $12)=$ & .1369 & DRI & $12)=$ & .0346 & RS ( & $12)=$ & 1.0000 & $\mathrm{R}($ & $44)=$ & .6902 & DRP & $44)=$ & .0346 & $\operatorname{RS}(44)=$ & 1.0000 \\
\hline Ri & $13)=$ & .1542 & DR( & $13)=$ & .0346 & RS ( & $13)=$ & 1.0000 & $\mathrm{R}($ & $45)=$ & .7075 & DR\& & $45)=$ & .0346 & $\operatorname{RS}(45)=$ & 1.0000 \\
\hline Ri & $14)=$ & .1715 & DRI & $14)=$ & .0346 & RS ( & $14)=$ & 1.0000 & $R($ & $46)=$ & .7248 & $D R($ & $46)=$ & .0346 & $\operatorname{RS}(46)=$ & 1.0000 \\
\hline Ri & $15)=$ & .1888 & DRI & $15)=$ & .0346 & RS? & $15)=$ & 1.0000 & Ri & $47)=$ & .7420 & $\mathrm{DR} /$ & $47)=$ & .0346 & $\operatorname{RS}(47)=$ & 1.0000 \\
\hline 21 & $16)=$ & .2061 & DRI & $16)=$ & .0346 & RS ( & $16)=$ & 1.0000 & $\mathrm{R} \ell$ & $48)=$ & .7593 & DRI & $48)=$ & .0346 & $R S(48)=$ & 1.0000 \\
\hline Ri & $17)=$ & .2234 & DR( & $17)=$ & .0346 & RS ( & 17) $=$ & 1.0000 & $\mathrm{R} \ell$ & $49)=$ & .7766 & DR( & $49)=$ & .0346 & $\operatorname{RS}(49)=$ & 1.0000 \\
\hline $\mathrm{R}$ ( & $18)=$ & .2407 & DR( & $18)=$ & .0346 & RS ( & $18)=$ & 1.0000 & $R($ & $50)=$ & .7939 & DRI & $50)=$ & .0346 & $\operatorname{RS}(50)=$ & 1.0000 \\
\hline Ri & $19)=$ & .2580 & DR( & $19)=$ & .0346 & RS 1 & $19)=$ & 1.0000 & $\mathrm{R}($ & $51)=$ & .8112 & DR( & $51)=$ & .0346 & $R S(51)=$ & 1.0000 \\
\hline Ri & $201=$ & .2752 & $\mathrm{DR}($ & $20)=$ & .0346 & RSI & $20)=$ & 1.0000 & ( & $52)=$ & .8285 & DR( & $52)=$ & .0346 & $\operatorname{RS}(52)=$ & 1.0000 \\
\hline $\mathrm{R}($ & $21)=$ & .2925 & $\mathrm{DR}($ & $21)=$ & .0346 & RS ( & $21)=$ & 1.0000 & $\mathrm{R}($ & $53)=$ & .8458 & DR! & $53)=$ & .0346 & $\operatorname{RS}(53)=$ & 1.0000 \\
\hline Ri & $22)=$ & .3098 & $\mathrm{DR}($ & $22)=$ & .0346 & RS ( & $22)=$ & 1.0000 & 1 & $54)=$ & .8631 & $\mathrm{R} \ell$ & $54)=$ & .0346 & $\operatorname{RS}(54)=$ & 1.0000 \\
\hline Ri & $231=$ & .3271 & $\mathrm{DR}$ ( & $23)=$ & .0346 & RS ( & $23)=$ & 1.0000 & $\mathrm{R}$ l & $55)=$ & .8804 & DRC & $55)=$ & .0346 & $\operatorname{RS}(55)=$ & 1.0000 \\
\hline Ri & $24)=$ & .3444 & DRC & $24)=$ & .0346 & RS ( & $24)=$ & 1.0000 & R( & $56)=$ & .8977 & $\mathrm{DR}($ & $56)=$ & .0346 & $\operatorname{RS}(56)=$ & 1.0000 \\
\hline$R<$ & $25)=$ & .3617 & DRC & $25)=$ & .0346 & $\mathrm{RS}($ & $25)=$ & 1.0000 & ( & $57)=$ & .9149 & DRC & $57)=$ & .0346 & $\operatorname{RS}(57)=$ & 1.0000 \\
\hline Ri & $261=$ & .3790 & DRS & $26)=$ & .0346 & RS ( & $26)=$ & 1.0000 & $R($ & $58)=$ & .9322 & DRI & $58)=$ & .0346 & $R S(58)=$ & 1.0000 \\
\hline $\mathrm{R}($ & $27)=$ & .3963 & DR ( & $27)=$ & .0346 & RS ( & $27)=$ & 1.0000 & $\mathrm{R}($ & $59)=$ & .9495 & DR( & $59)=$ & .0346 & $\operatorname{RS}(59)=$ & 1.0000 \\
\hline$R($ & $28)=$ & .4136 & DRI & $28)=$ & .0346 & RS ( & $28)=$ & 1.0000 & $\mathrm{R}($ & $60)=$ & .9668 & $R($ & $60)=$ & .0346 & $\operatorname{RS}(60)=$ & 1.0000 \\
\hline $\mathrm{R}$ ( & $29)=$ & .4308 & DRI & $29)=$ & .0346 & RS ( & $29)=$ & 1.0000 & $\mathrm{R}($ & $61)=$ & .9841 & DRC & $61)=$ & .0252 & $\operatorname{RS}(61)=$ & 1.0000 \\
\hline $\mathrm{R}($ & $30)=$ & .4481 & DR( & $30)=$ & .0346 & RS & $30)=$ & 1.0000 & $\mathrm{R}($ & $62)=$ & .9920 & DRI & $62)=$ & .0159 & $\operatorname{RS}(62)=$ & 1.0000 \\
\hline RI & $31)=$ & .4654 & DRI & $311=$ & .0346 & RS ( & $31)=$ & 1.0000 & $\mathrm{R}($ & $63)=$ & 1.0000 & $\mathrm{DR}($ & $63)=$ & .0080 & $\operatorname{RS}(63)=$ & 1.0000 \\
\hline 8 & $32)=$ & .4827 & $\mathrm{DR}($ & $32)=$ & .0346 & RS ( & $32)=$ & 1.0000 & $\mathrm{Rl}$ & $64)=$ & 1.0000 & DRl & $64)=$ & .0000 & $\operatorname{RS}(64)=$ & 1.0000 \\
\hline
\end{tabular}

INLET AND JET MASS FLOW RATES

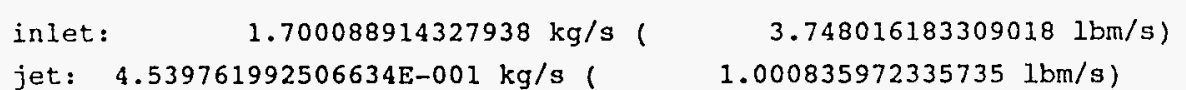

E/O ratio: $\quad 1.378117887028782$ 
PROGRAM CALCULATION RESULTS

INLET CONDITION AND NON-DIMENSIONAL PARAMETERS

\begin{tabular}{|c|c|c|c|c|c|c|c|c|c|c|c|c|}
\hline UGO = & 29.300 & ULO $=$ & 25.000 & TO & 974.000 & $\mathrm{TINF}=1$ & 4.000 & PGO & & $.5776 E+06$ & $A R I E=$ & .0000 \\
\hline$B E T A O=$ & .0000 & RLMO $=$ & $.3430 E-04$ & DNDO $=$ & $.6600 E+08$ & TKO $=$ & 4.292 & EPSO & $=$ & $.184 E+02$ & RLNE $=$ & 1.0000 \\
\hline EO & .3680 & YOXO $=$ & .0000 & UG0 $=$ & 29.300 & $\mathrm{WGO}=$ & .000 & TLO $=$ & $=27$ & 3.000 & THETAO $=$ & 1.0000 \\
\hline
\end{tabular}

TPO $=.200 \mathrm{E}-01 \quad$ DTO $=.100 \mathrm{E}-01 \quad$ DNSTO $=1.110$

REYNOLDS $=.8312 \mathrm{E}+06$

$\mathrm{GD}$ NUMBER $=.113 \mathrm{E}-01$

$\mathrm{RFG}=.1969 \mathrm{E}-01$

EULER $=605.9$
SCHMIDT $=1.174$

ECKERT $=.4067 \mathrm{E}-03$

$\mathrm{EPSR}=.5000 \mathrm{E}-02$

DNULO $=2.246$
GC NUMBER $=.807 \mathrm{E}-02$

DAMKHOLER $=.28301 E+06$

TAUR

DNUCO
$=.3349 \mathrm{E}-01$

$=4.952$
GV NUMBER $=.889 \mathrm{E}-02$

HEAT VALUE $=.8389 \mathrm{E}+07$

F/A RATIO $=1.3781$

YFOP RATIO $=.57950$

TYPICAL ITERATION RESULT FOR GAS FLOW FIELD

\begin{tabular}{|c|c|c|c|c|c|c|c|c|c|c|c|}
\hline LLK & $\operatorname{sMX}$ & IMX & JMX & AVEB & $U(I, J)$ & $V(I, J)$ & $P(I, J)$ & $\operatorname{DNST}(I, J)$ & $T(I, J)$ & & \\
\hline 1 & $.5703 \mathrm{E}-04$ & 40 & 4 & $.7598 \mathrm{E}-06$ & $.3583 E+01$ & $.5434 \mathrm{E}+00$ & $.2555 \mathrm{E}-01$ & $.1001 E+01$ & $.9765 \mathrm{E}+00$ & & \\
\hline & & & & & & & & & & & \\
\hline 200 & $.1958 \mathrm{E}-06$ & 18 & 4 & $.7020 \mathrm{E}-08$ & $.3986 \mathrm{E}-01$ & $.1906 \mathrm{E}+00$ & $.1484 \mathrm{E}-02$ & $.1093 \mathrm{E}+01$ & $.7291 E+00$ & & \\
\hline & TYPI & CAL & TERA & N RESULT FO & TWO-PHASE & WW FIELD & & & & & \\
\hline LLK & SMX & IMX & JMX & AVEB & $\mathrm{U}(I, J)$ & $V(I, J)$ & $\mathrm{P}(I, J)$ & $\operatorname{DNST}(I, J)$ & $\mathrm{T}(I, J)$ & & \\
\hline 1 & $.1979 \mathrm{E}-06$ & 18 & 62 & $.6921 E-08$ & $.3583 \mathrm{E}-01$ & $-.1914 \mathrm{E}+00$ & $.1486 \mathrm{E}-02$ & $.1095 E+01$ & $.7277 E+00$ & & \\
\hline & $.9785 \mathrm{E}-02$ & 15 & 2 & $.3438 \mathrm{E}-03$ & $-.2876 \mathrm{E}+00$ & $.3812 E+00$ & $.9530 \mathrm{E}-05$ & $.6088 E+00$ & $.2728 E+00$ & $.0000 \mathrm{E}+00$ & $.1151 \mathrm{E}+01$ \\
\hline & $.1264 \mathrm{E}-01$ & 12 & 3 & $.5259 \mathrm{E}-03$ & $-.4502 E+00$ & $.1850 \mathrm{E}+00$ & $.1151 \mathrm{E}-04$ & $.7600 \mathrm{E}+00$ & $.2529 E+00$ & $.0000 E+00$ & $.1264 E+01$ \\
\hline$\ldots$ & & & & & & & & & & & \\
\hline 352 & $.1736 \mathrm{E}-08$ & 22 & 56 & $.9914 \mathrm{E}-10$ & $-.4666 \mathrm{E}+00$ & $-.1142 \mathrm{E}+01$ & $-.4477 E-02$ & $.1213 \mathrm{E}+01$ & $.6067 E+00$ & & \\
\hline & $.3981 \mathrm{E}-06$ & 10 & 3 & $.1754 \mathrm{E}-07$ & $-.1650 \mathrm{E}+00$ & $.1333 E+00$ & $.9984 \mathrm{E}-05$ & $.1324 \mathrm{E}+00$ & $.4862 \mathrm{E}+00$ & $.0000 E+00$ & $.1009 E+01$ \\
\hline & $.3174 \mathrm{E}-06$ & 10 & 29 & $.1595 \mathrm{E}-07$ & $-.7060 \mathrm{E}-02$ & $-.3427 \mathrm{E}+00$ & $.1641 \mathrm{E}-04$ & $.4220 \mathrm{E}+00$ & $.4911 \mathrm{E}+00$ & $.0000 \mathrm{E}+00$ & $.1009 E+01$ \\
\hline
\end{tabular}




\section{Some Results of the Sample Calculation}

"RESULTS OVER FLOWFIELD TIME STEP $=0$ TIME $=\quad .000 "$

"GAS PRESSURE FIELD, P(I,J)TSTEP O"

"GAS TEMPERATURE/TINF, TINF $=1974 . \quad \mathrm{K}=3553 . \quad \mathrm{R}, \mathrm{TIME}$ STEP $0 "$

$\begin{array}{lllllllllllllllll}X & R & .000 & .008 & .033 & .068 & .102 & .137 & .172 & .206 & .241 & .275 & .310\end{array}$

$\begin{array}{llllllllllll}.00 & 1.000 \mathrm{E}+00 & 1.000 \mathrm{E}+00 & 1.000 \mathrm{E}+00 & 1.000 \mathrm{E}+00 & 1.000 \mathrm{E}+00 & 1.000 \mathrm{E}+00 & 1.000 \mathrm{E}+00 & 1.000 \mathrm{E}+00 & 1.000 \mathrm{E}+00 & 1.000 \mathrm{E}+00 & 1.000 \mathrm{E}+00\end{array}$

.02 9.993E-01 9.993E-01 9.992E-01 9.992E-01 9.992E-01 9.992E-01 9.991E-01 9.991E-01 9.991E-01 9.991E-01 9.991E-01 .07 9.983E-01 9.983E-01 9.983E-01 9.982E-01 9.982E-01 9.981E-01 9.981E-01 9.980E-01 9.980E-01 9.980E-01 9.980E-01 $.129 .974 \mathrm{E}-01 \quad 9.974 \mathrm{E}-01 \quad 9.973 \mathrm{E}-01 \quad 9.972 \mathrm{E}-01 \quad 9.971 \mathrm{E}-01 \quad 9.971 \mathrm{E}-01 \quad 9.970 \mathrm{E}-01 \quad 9.970 \mathrm{E}-01 \quad 9.970 \mathrm{E}-019.970 \mathrm{E}-01 \quad 9.970 \mathrm{E}-01$

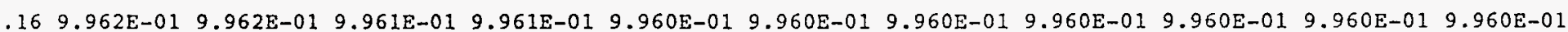
$.219 .945 \mathrm{E}-01 \quad 9.945 \mathrm{E}-01 \quad 9.946 \mathrm{E}-01 \quad 9.950 \mathrm{E}-01 \quad 9.954 \mathrm{E}-01 \quad 9.956 \mathrm{E}-01 \quad 9.956 \mathrm{E}-01 \quad 9.956 \mathrm{E}-01 \quad 9.955 \mathrm{E}-01 \quad 9.954 \mathrm{E}-01 \quad 9.954 \mathrm{E}-01$

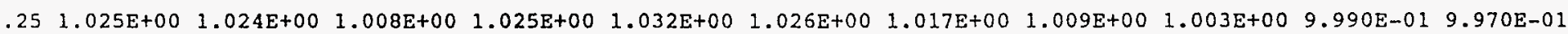
$\begin{array}{llllllllllll}.30 & 1.079 \mathrm{E}+00 & 1.054 \mathrm{E}+00 & 6.460 \mathrm{E}-01 & 6.673 \mathrm{E}-01 & 7.901 \mathrm{E}-01 & 9.209 \mathrm{E}-01 & 1.025 \mathrm{E}+00 & 1.071 \mathrm{E}+00 & 1.065 \mathrm{E}+00 \quad 1.042 \mathrm{E}+00 \quad 1.021 \mathrm{E}+00\end{array}$

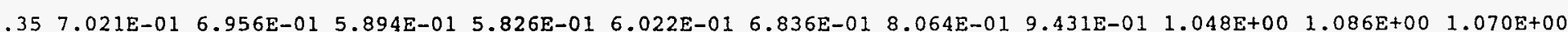
$\begin{array}{lllllllllllll}.39 & 4.375 \mathrm{E}-01 & 4.429 \mathrm{E}-01 & 5.309 \mathrm{E}-01 & 5.630 \mathrm{E}-01 & 5.712 \mathrm{E}-01 & 5.971 \mathrm{E}-01 & 6.732 \mathrm{E}-01 & 7.865 \mathrm{E}-01 & 9.277 \mathrm{E}-01 \quad 1.048 \mathrm{E}+00 & 1.106 \mathrm{E}+00\end{array}$ $.44 \quad 4.354 \mathrm{E}-01 \quad 4.420 \mathrm{E}-01 \quad 5.505 \mathrm{E}-01 \quad 5.939 \mathrm{E}-01 \quad 6.067 \mathrm{E}-01 \quad 6.052 \mathrm{E}-01 \quad 6.327 \mathrm{E}-01 \quad 7.097 \mathrm{E}-01 \quad 8.263 \mathrm{E}-01 \quad 9.718 \mathrm{E}-01 \quad 1.095 \mathrm{E}+00$ $.494 .325 \mathrm{E}-01 \quad 4.402 \mathrm{E}-01 \quad 5.659 \mathrm{E}-01 \quad 6.209 \mathrm{E}-01 \quad 6.371 \mathrm{E}-01 \quad 6.311 \mathrm{E}-01 \quad 6.327 \mathrm{E}-01 \quad 6.772 \mathrm{E}-01 \quad 7.758 \mathrm{E}-01 \quad 9.101 \mathrm{E}-01 \quad 1.063 \mathrm{E}+00$ $\begin{array}{llllllllllll}.53 & 4.284 \mathrm{E}-01 & 4.371 \mathrm{E}-01 & 5.790 \mathrm{E}-01 & 6.450 \mathrm{E}-01 & 6.609 \mathrm{E}-01 & 6.552 \mathrm{E}-01 & 6.438 \mathrm{E}-01 & 6.678 \mathrm{E}-01 & 7.526 \mathrm{E}-01 & 8.750 \mathrm{E}-01 & 1.037 \mathrm{E}+00\end{array}$

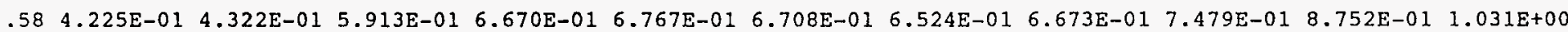
$.624 .143 \mathrm{E}-01 \quad 4.253 \mathrm{E}-01 \quad 6.058 \mathrm{E}-01 \quad 6.899 \mathrm{E}-01 \quad 6.837 \mathrm{E}-01 \quad 6.674 \mathrm{E}-01 \quad 6.562 \mathrm{E}-01 \quad 6.757 \mathrm{E}-01 \quad 7.604 \mathrm{E}-01 \quad 8.966 \mathrm{E}-01 \quad 1.043 \mathrm{E}+00$ $\begin{array}{llllllllllllll}.65 & 4.067 \mathrm{E}-01 & 4.202 \mathrm{E}-01 & 6.430 \mathrm{E}-01 & 7.292 \mathrm{E}-01 & 6.843 \mathrm{E}-01 & 6.659 \mathrm{E}-01 & 6.573 \mathrm{E}-01 & 6.781 \mathrm{E}-01 & 7.643 \mathrm{E}-01 & 9.036 \mathrm{E}-01 & 1.047 \mathrm{E}+00\end{array}$ $\begin{array}{lllllllllllllll}.66 & 1.520 \mathrm{E}-01 & 4.106 \mathrm{E}-01 & 7.073 \mathrm{E}-01 & 7.393 \mathrm{E}-01 & 6.859 \mathrm{E}-01 & 6.655 \mathrm{E}-01 & 6.582 \mathrm{E}-01 & 6.803 \mathrm{E}-01 & 7.695 \mathrm{E}-01 & 9.156 \mathrm{E}-01 \quad 1.054 \mathrm{E}+00\end{array}$ $\begin{array}{llllllllllllllllll}.66 & 1.520 \mathrm{E}-01 & 5.134 \mathrm{E}-01 & 7.596 \mathrm{E}-01 & 7.424 \mathrm{E}-01 & 6.869 \mathrm{E}-01 & 6.653 \mathrm{E}-01 & 6.590 \mathrm{E}-01 & 6.827 \mathrm{E}-01 & 7.754 \mathrm{E}-01 & 9.306 \mathrm{E}-01 & 1.065 \mathrm{E}+00\end{array}$ $\begin{array}{llllllllllll}.56 & 1.520 \mathrm{E}-01 & 6.559 \mathrm{E}-01 & 7.959 \mathrm{E}-01 & 7.452 \mathrm{E}-01 & 6.882 \mathrm{E}-01 & 6.652 \mathrm{E}-01 & 6.598 \mathrm{E}-01 & 6.847 \mathrm{E}-01 & 7.801 \mathrm{E}-01 & 9.425 \mathrm{E}-01 & 1.085 \mathrm{E}+00\end{array}$ $.678 .710 \mathrm{E}-01$ 8.678E-01 8.157E-01 7.492E-01 6.902E-01 6.654E-01 6.618E-01 6.887E-01 $7.869 \mathrm{E}-01 \quad 9.560 \mathrm{E}-01 \quad 1.124 \mathrm{E}+00$ $\begin{array}{llllllllllllll}.68 & 8.815 \mathrm{E}-01 & 8.783 \mathrm{E}-01 & 8.255 \mathrm{E}-01 & 7.558 \mathrm{E}-01 & 6.943 \mathrm{E}-01 & 6.664 \mathrm{E}-01 & 6.641 \mathrm{E}-01 & 6.928 \mathrm{E}-01 & 7.924 \mathrm{E}-01 & 9.649 \mathrm{E}-01 \quad 1.148 \mathrm{E}+00\end{array}$ $.608 .898 \mathrm{E}-01 \quad 8.866 \mathrm{E}-01 \quad 8.340 \mathrm{E}-01 \quad 7.635 \mathrm{E}-01 \quad 7.001 \mathrm{E}-01 \quad 6.701 \mathrm{E}-01 \quad 6.705 \mathrm{E}-01 \quad 7.024 \mathrm{E}-01 \quad 8.036 \mathrm{E}-01 \quad 9.786 \mathrm{E}-01 \quad 1.177 \mathrm{E}+00$ $\begin{array}{lllllllllll}.71 & 9.020 \mathrm{E}-01 & 8.989 \mathrm{E}-01 & 8.471 \mathrm{E}-01 & 7.774 \mathrm{E}-01 & 7.127 \mathrm{E}-01 & 6.810 \mathrm{E}-01 & 6.839 \mathrm{E}-01 & 7.203 \mathrm{E}-01 & 8.229 \mathrm{E}-01 \quad 9.980 \mathrm{E}-01 \quad 1.202 \mathrm{E}+00\end{array}$ $\begin{array}{llllllllllll}.74 & 9.144 \mathrm{E}-01 & 9.115 \mathrm{E}-01 & 8.631 \mathrm{E}-01 & 7.972 \mathrm{E}-01 & 7.339 \mathrm{E}-01 & 7.026 \mathrm{E}-01 & 7.076 \mathrm{E}-01 & 7.492 \mathrm{E}-01 & 8.532 \mathrm{E}-01 & 1.026 \mathrm{E}+00 & 1.229 \mathrm{E}+00\end{array}$ 
$\begin{array}{lllllllllll}.77 & 9.272 \mathrm{E}-01 & 9.245 \mathrm{E}-01 & 8.814 \mathrm{E}-01 & 8.224 \mathrm{E}-01 & 7.642 \mathrm{E}-01 & 7.356 \mathrm{E}-01 & 7.435 \mathrm{E}-01 & 7.903 \mathrm{E}-01 & 8.958 \mathrm{E}-01 & 1.065 \mathrm{E}+00 \quad 1.259 \mathrm{E}+00\end{array}$ $.829 .413 \mathrm{E}-0 \mathrm{I} \quad 9.390 \mathrm{E}-01 \quad 9.019 \mathrm{E}-01 \quad 8.519 \mathrm{E}-01 \quad 8.022 \mathrm{E}-01 \quad 7.786 \mathrm{E}-01 \quad 7.903 \mathrm{E}-01 \quad 8.423 \mathrm{E}-01 \quad 9.493 \mathrm{E}-01 \quad 1.113 \mathrm{E}+00 \quad 1.289 \mathrm{E}+00$

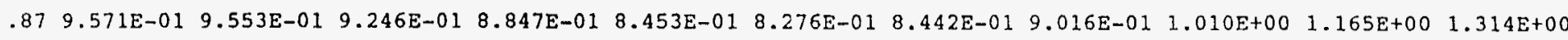
$.939 .747 \mathrm{E}-01$ 9.732E-01 9.490E-01 9.190E-01 8.903E-01 8.788E-01 9.009E-01 9.639E-01 $1.073 \mathrm{E}+00 \quad 1.216 \mathrm{E}+00 \quad 1.330 \mathrm{E}+00$

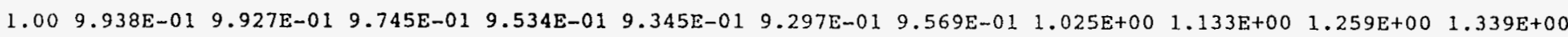
$\begin{array}{llllllllll}1.07 & 1.014 \mathrm{E}+00 & 1.014 \mathrm{E}+00 & 1.001 \mathrm{E}+00 & 9.876 \mathrm{E}-01 & 9.772 \mathrm{E}-01 & 9.793 \mathrm{E}-01 & 1.011 \mathrm{E}+00 & 1.083 \mathrm{E}+00 & 1.187 \mathrm{E}+00 \quad 1.292 \mathrm{E}+00 \quad 1.342 \mathrm{E}+00\end{array}$ $\begin{array}{lllllllllll}1.16 & 1.037 \mathrm{E}+00 & 1.036 \mathrm{E}+00 & 1.028 \mathrm{E}+00 & 1.021 \mathrm{E}+00 & 1.018 \mathrm{E}+00 & 1.027 \mathrm{E}+00 & 1.063 \mathrm{E}+00 & 1.134 \mathrm{E}+00 & 1.230 \mathrm{E}+00 \quad 1.313 \mathrm{E}+00 \quad 1.341 \mathrm{E}+00\end{array}$ $\begin{array}{llllllllllll}1.25 & 1.060 \mathrm{E}+00 & 1.060 \mathrm{E}+00 & 1.057 \mathrm{E}+00 & 1.055 \mathrm{E}+00 & 1.059 \mathrm{E}+00 & 1.074 \mathrm{E}+00 & 1.112 \mathrm{E}+00 & 1.180 \mathrm{E}+00 & 1.263 \mathrm{E}+00 & 1.327 \mathrm{E}+00 & 1.340 \mathrm{E}+00\end{array}$ $\begin{array}{llllllllll}1.35 & 1.087 \mathrm{E}+00 & 1.087 \mathrm{E}+00 & 1.087 \mathrm{E}+00 & 1.089 \mathrm{E}+00 & 1.098 \mathrm{E}+00 & 1.118 \mathrm{E}+00 & 1.156 \mathrm{E}+00 & 1.217 \mathrm{E}+00 & 1.287 \mathrm{E}+00 \quad 1.335 \mathrm{E}+00 \quad 1.340 \mathrm{E}+00\end{array}$

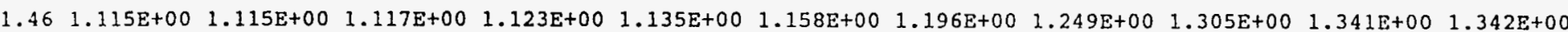

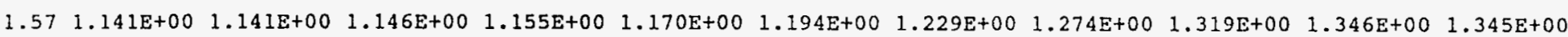
$\begin{array}{llllllllll}1.69 & 1.165 \mathrm{E}+00 & 1.166 \mathrm{E}+00 & 1.172 \mathrm{E}+00 & 1.183 \mathrm{E}+00 & 1.200 \mathrm{E}+00 & 1.224 \mathrm{E}+00 & 1.256 \mathrm{E}+00 & 1.294 \mathrm{E}+00 & 1.330 \mathrm{E}+00 \quad 1.352 \mathrm{E}+00 \quad 1.349 \mathrm{E}+00\end{array}$ $\begin{array}{lllllllllll}1.81 & 1.188 \mathrm{E}+00 & 1.188 \mathrm{E}+00 & 1.196 \mathrm{E}+00 & 1.209 \mathrm{E}+00 & 1.226 \mathrm{E}+00 & 1.249 \mathrm{E}+00 & 1.278 \mathrm{E}+00 & 1.311 \mathrm{E}+00 & 1.341 \mathrm{E}+00 \quad 1.358 \mathrm{E}+00 & 1.356 \mathrm{E}+00\end{array}$ $\begin{array}{llllllllllll}1.93 & 1.209 \mathrm{E}+00 & 1.209 \mathrm{E}+00 & 1.218 \mathrm{E}+00 & 1.231 \mathrm{E}+00 & 1.249 \mathrm{E}+00 & 1.271 \mathrm{E}+00 & 1.297 \mathrm{E}+00 & 1.326 \mathrm{E}+00 & 1.351 \mathrm{E}+00 & 1.366 \mathrm{E}+00 & 1.364 \mathrm{E}+00\end{array}$ $2.041 .228 \mathrm{E}+00 \quad 1.229 \mathrm{E}+00 \quad 1.238 \mathrm{E}+00 \quad 1.252 \mathrm{E}+00 \quad 1.269 \mathrm{E}+00 \quad 1.290 \mathrm{E}+00 \quad 1.314 \mathrm{E}+00 \quad 1.339 \mathrm{E}+00 \quad 1.360 \mathrm{E}+00 \quad 1.373 \mathrm{E}+00 \quad 1.371 \mathrm{E}+00$ $\begin{array}{llllllllllll}2.16 & 1.246 \mathrm{E}+00 & 1.246 \mathrm{E}+00 & 1.257 \mathrm{E}+00 & 1.270 \mathrm{E}+00 & 1.286 \mathrm{E}+00 & 1.306 \mathrm{E}+00 & 1.328 \mathrm{E}+00 & 1.350 \mathrm{E}+00 & 1.369 \mathrm{E}+00 & 1.380 \mathrm{E}+00 & 1.379 \mathrm{E}+00\end{array}$ $2.28 \quad 1.262 \mathrm{E}+00 \quad 1.262 \mathrm{E}+00 \quad 1.273 \mathrm{E}+00 \quad 1.286 \mathrm{E}+00 \quad 1.302 \mathrm{E}+00 \quad 1.320 \mathrm{E}+00 \quad 1.340 \mathrm{E}+00 \quad 1.360 \mathrm{E}+00 \quad 1.377 \mathrm{E}+00 \quad 1.387 \mathrm{E}+00 \quad 1.387 \mathrm{E}+00$ $2.40 \quad 1.276 \mathrm{E}+00 \quad 1.277 \mathrm{E}+00 \quad 1.287 \mathrm{E}+00 \quad 1.300 \mathrm{E}+00 \quad 1.315 \mathrm{E}+00 \quad 1.332 \mathrm{E}+00 \quad 1.351 \mathrm{E}+00 \quad 1.369 \mathrm{E}+00 \quad 1.384 \mathrm{E}+00 \quad 1.394 \mathrm{E}+00 \quad 1.395 \mathrm{E}+00$

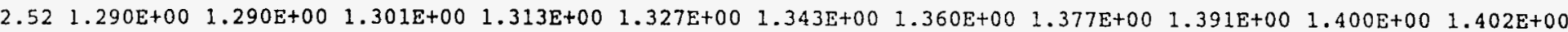
$\begin{array}{lllllllllll}2.63 & 1.302 E+00 & 1.302 E+00 & 1.312 E+00 & 1.324 E+00 & 1.338 E+00 & 1.353 E+00 & 1.369 E+00 & 1.384 E+00 & 1.397 E+00 \quad 1.406 E+00 & 1.408 E+00\end{array}$ $2.75 \quad 1.312 \mathrm{E}+00 \quad 1.313 \mathrm{E}+00 \quad 1.323 \mathrm{E}+00 \quad 1.334 \mathrm{E}+00 \quad 1.347 \mathrm{E}+00 \quad 1.361 \mathrm{E}+00 \quad 1.376 \mathrm{E}+00 \quad 1.390 \mathrm{E}+00 \quad 1.403 \mathrm{E}+00 \quad 1.411 \mathrm{E}+00 \quad 1.414 \mathrm{E}+00$

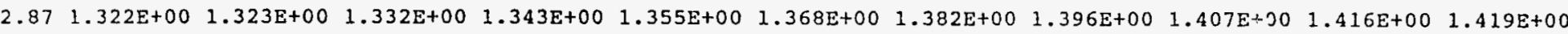
$2.99 \quad 1.331 \mathrm{E}+00 \quad 1.332 \mathrm{E}+00 \quad 1.341 \mathrm{E}+00 \quad 1.351 \mathrm{E}+00 \quad 1.363 \mathrm{E}+00 \quad 1.375 \mathrm{E}+00 \quad 1.388 \mathrm{E}+00 \quad 1.400 \mathrm{E}+00 \quad 1.411 \mathrm{E}+00 \quad 1.420 \mathrm{E}+00 \quad 1.424 \mathrm{E}+00$ $\begin{array}{llllllllll}3.11 & 1.339 \mathrm{E}+00 & 1.340 \mathrm{E}+00 & 1.348 \mathrm{E}+00 & 1.358 \mathrm{E}+00 & 1.369 \mathrm{E}+00 & 1.381 \mathrm{E}+00 & 1.393 \mathrm{E}+00 & 1.405 \mathrm{E}+00 & 1.415 \mathrm{E}+00 \quad 1.423 \mathrm{E}+00 \quad 1.428 \mathrm{E}+00\end{array}$

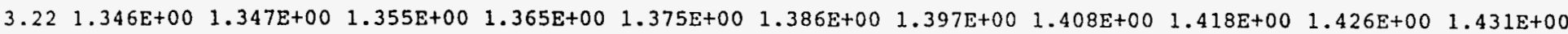

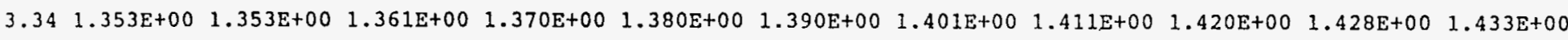

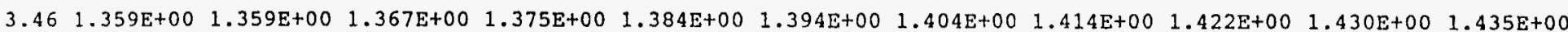

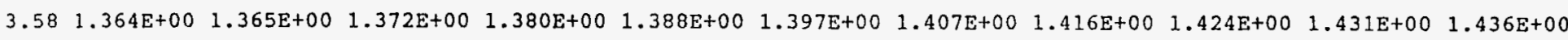

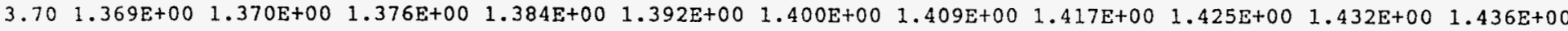
$\begin{array}{llllllllll}3.81 & 1.374 \mathrm{E}+00 & 1.374 \mathrm{E}+00 & 1.381 \mathrm{E}+00 & 1.388 \mathrm{E}+00 & 1.395 \mathrm{E}+00 & 1.403 \mathrm{E}+00 & 1.411 \mathrm{E}+00 & 1.419 \mathrm{E}+00 & 1.426 \mathrm{E}+00 \quad 1.432 \mathrm{E}+00 \quad 1.437 \mathrm{E}+00\end{array}$

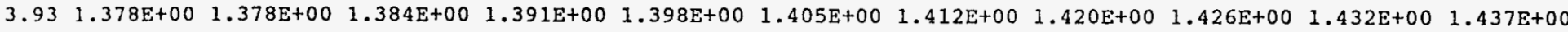

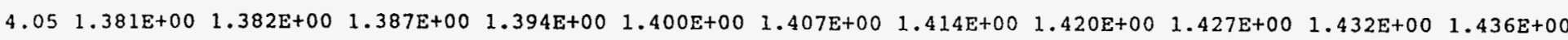
$4.11 \quad 1.383 E+00 \quad 1.383 E+00 \quad 1.389 E+00 \quad 1.395 E+00 \quad 1.401 E+00 \quad 1.408 E+00 \quad 1.414 E+00 \quad 1.421 E+00 \quad 1.427 E+00 \quad 1.432 E+00 \quad 1.436 E+00$

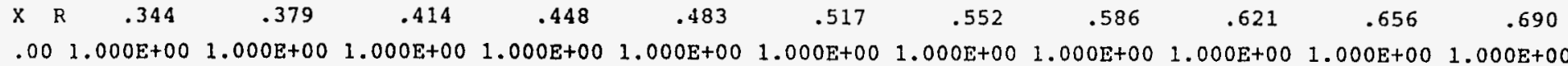




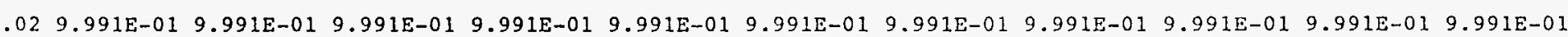
.07 9.980E-01 9.980E-01 9.980E-01 9.981E-01 9.981E-01 9.981E-01 9.981E-01 9.980E-01 9.980E-01 9.980E-01 9.980E-01

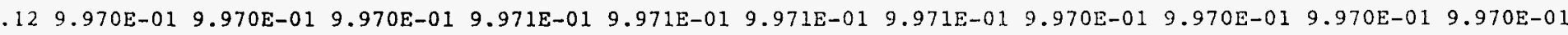
. 16 9.961E-01 9.961E-01 9.961E-01 9.961E-01 9.962E-01 9.962E-01 9.961E-01 9.961E-01 9.961E-01 $9.961 \mathrm{E}-019.960 \mathrm{E}-01$ $.219 .954 \mathrm{E}-01 \quad 9.954 \mathrm{E}-01 \quad 9.954 \mathrm{E}-01 \quad 9.954 \mathrm{E}-01 \quad 9.954 \mathrm{E}-01 \quad 9.954 \mathrm{E}-01 \quad 9.954 \mathrm{E}-01 \quad 9.954 \mathrm{E}-01 \quad 9.954 \mathrm{E}-019.954 \mathrm{E}-019.954 \mathrm{E}-01$ $.259 .961 \mathrm{E}-01 \quad 9.956 \mathrm{E}-01 \quad 9.953 \mathrm{E}-01 \quad 9.951 \mathrm{E}-01 \quad 9.950 \mathrm{E}-01 \quad 9.950 \mathrm{E}-01 \quad 9.951 \mathrm{E}-019.953 \mathrm{E}-019.956 \mathrm{E}-019.961 \mathrm{E}-019.970 \mathrm{E}-01$ $.301 .007 \mathrm{E}+00 \quad 1.001 \mathrm{E}+00 \quad 9.976 \mathrm{E}-01 \quad 9.962 \mathrm{E}-01 \quad 9.956 \mathrm{E}-01 \quad 9.956 \mathrm{E}-01 \quad 9.962 \mathrm{E}-01 \quad 9.976 \mathrm{E}-01 \quad 1.001 \mathrm{E}+00 \quad 1.007 \mathrm{E}+00 \quad 1.021 \mathrm{E}+00$ $\begin{array}{lllllllllll}.35 & 1.039 \mathrm{E}+00 & 1.017 \mathrm{E}+00 & 1.005 \mathrm{E}+00 & 1.000 \mathrm{E}+00 & 9.981 \mathrm{E}-01 & 9.981 \mathrm{E}-01 & 1.000 \mathrm{E}+00 & 1.005 \mathrm{E}+00 & 1.017 \mathrm{E}+00 \quad 1.039 \mathrm{E}+00 \quad 1.070 \mathrm{E}+00\end{array}$ $\begin{array}{lllllllllll}.39 & 1.084 \mathrm{E}+00 & 1.045 \mathrm{E}+00 & 1.020 \mathrm{E}+00 & 1.008 \mathrm{E}+00 & 1.004 \mathrm{E}+00 & 1.004 \mathrm{E}+00 & 1.008 \mathrm{E}+00 & 1.020 \mathrm{E}+00 & 1.045 \mathrm{E}+00 \quad 1.084 \mathrm{E}+00 \quad 1.106 \mathrm{E}+00\end{array}$ $\begin{array}{llllllllll}.44 & 1.124 \mathrm{E}+00 & 1.079 \mathrm{E}+00 & 1.041 \mathrm{E}+00 & 1.021 \mathrm{E}+00 & 1.013 \mathrm{E}+00 & 1.013 \mathrm{E}+00 & 1.021 \mathrm{E}+00 & 1.041 \mathrm{E}+00 & 1.079 \mathrm{E}+00 \quad 1.124 \mathrm{E}+00 \quad 1.095 \mathrm{E}+00\end{array}$

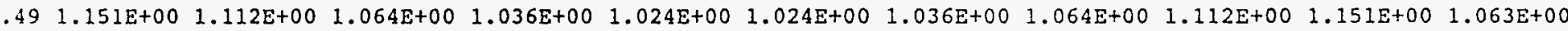
$\begin{array}{lllllllllll}.53 & 1.167 E+00 & 1.141 \mathrm{E}+00 & 1.087 \mathrm{E}+00 & 1.054 \mathrm{E}+00 & 1.038 \mathrm{E}+00 & 1.038 \mathrm{E}+00 & 1.054 \mathrm{E}+00 & 1.087 \mathrm{E}+00 & 1.141 \mathrm{E}+00 \quad 1.167 \mathrm{E}+00 \quad 1.037 \mathrm{E}+00\end{array}$

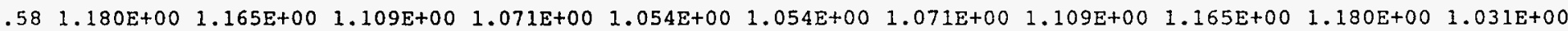
$\begin{array}{lllllllllll}.62 & 1.195 \mathrm{E}+00 & 1.184 \mathrm{E}+00 & 1.129 \mathrm{E}+00 & 1.090 \mathrm{E}+00 & 1.071 \mathrm{E}+00 & 1.071 \mathrm{E}+00 & 1.090 \mathrm{E}+00 & 1.129 \mathrm{E}+00 & 1.184 \mathrm{E}+00 \quad 1.195 \mathrm{E}+00 \quad 1.043 \mathrm{E}+00\end{array}$

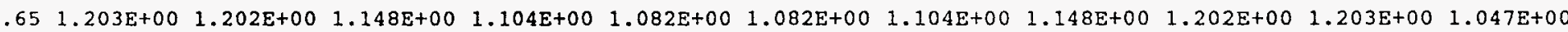

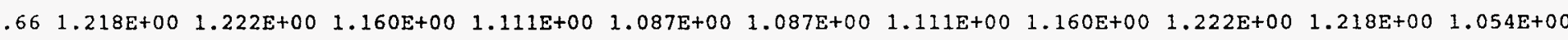
$\begin{array}{llllllllll}.66 & 1.233 \mathrm{E}+00 & 1.238 \mathrm{E}+00 & 1.169 \mathrm{E}+00 & 1.116 \mathrm{E}+00 & 1.091 \mathrm{E}+00 & 1.091 \mathrm{E}+00 & 1.116 \mathrm{E}+00 & 1.169 \mathrm{E}+00 & 1.238 \mathrm{E}+00 \quad 1.233 \mathrm{E}+00 \quad 1.065 \mathrm{E}+00\end{array}$ $\begin{array}{lllllllllll}.66 & 1.244 \mathrm{E}+00 & 1.249 \mathrm{E}+00 & 1.176 \mathrm{E}+00 & 1.120 \mathrm{E}+00 & 1.094 \mathrm{E}+00 & 1.094 \mathrm{E}+00 & 1.120 \mathrm{E}+00 & 1.176 \mathrm{E}+00 & 1.249 \mathrm{E}+00 \quad 1.244 \mathrm{E}+00 \quad 1.085 \mathrm{E}+00\end{array}$

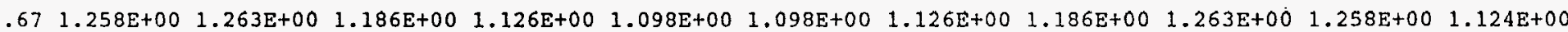
$\begin{array}{llllllllll}.68 & 1.268 \mathrm{E}+00 & 1.271 \mathrm{E}+00 & 1.193 \mathrm{E}+00 & 1.131 \mathrm{E}+00 & 1.102 \mathrm{E}+00 & 1.102 \mathrm{E}+00 & 1.131 \mathrm{E}+00 & 1.193 \mathrm{E}+00 & 1.271 \mathrm{E}+00 \quad 1.268 \mathrm{E}+00 \quad 1.148 \mathrm{E}+00\end{array}$ $\begin{array}{lllllllllll}.69 & 1.284 \mathrm{E}+00 & 1.279 \mathrm{E}+00 & 1.201 \mathrm{E}+00 & 1.139 \mathrm{E}+00 & 1.109 \mathrm{E}+00 & 1.109 \mathrm{E}+00 & 1.139 \mathrm{E}+00 & 1.201 \mathrm{E}+00 & 1.279 \mathrm{E}+00 & 1.284 \mathrm{E}+00 \quad 1.177 \mathrm{E}+00\end{array}$ $\begin{array}{lllllllllll}.71 & 1.309 \mathrm{E}+00 & 1.283 \mathrm{E}+00 & 1.209 \mathrm{E}+00 & 1.148 \mathrm{E}+00 & 1.118 \mathrm{E}+00 & 1.118 \mathrm{E}+00 & 1.148 \mathrm{E}+00 & 1.209 \mathrm{E}+00 & 1.283 \mathrm{E}+00 \quad 1.309 \mathrm{E}+00 \quad 1.202 \mathrm{E}+00\end{array}$ $\begin{array}{lllllllllll}.74 & 1.333 \mathrm{E}+00 & 1.285 \mathrm{E}+00 & 1.215 \mathrm{E}+00 & 1.158 \mathrm{E}+00 & 1.130 \mathrm{E}+00 & 1.130 \mathrm{E}+00 & 1.158 \mathrm{E}+00 & 1.215 \mathrm{E}+00 & 1.285 \mathrm{E}+00 \quad 1.333 \mathrm{E}+00 \quad 1.229 \mathrm{E}+00\end{array}$ $\begin{array}{llllllllll}.77 & 1.346 \mathrm{E}+00 & 1.285 \mathrm{E}+00 & 1.220 \mathrm{E}+00 & 1.169 \mathrm{E}+00 & 1.143 \mathrm{E}+00 & 1.143 \mathrm{E}+00 & 1.169 \mathrm{E}+00 & 1.220 \mathrm{E}+00 & 1.285 \mathrm{E}+00 \quad 1.346 \mathrm{E}+00 \quad 1.259 \mathrm{E}+00\end{array}$ $\begin{array}{lllllllllll}.82 & 1.347 \mathrm{E}+00 & 1.284 \mathrm{E}+00 & 1.226 \mathrm{E}+00 & 1.180 \mathrm{E}+00 & 1.157 \mathrm{E}+00 & 1.157 \mathrm{E}+00 & 1.180 \mathrm{E}+00 & 1.226 \mathrm{E}+00 & 1.284 \mathrm{E}+00 \quad 1.347 \mathrm{E}+00 \quad 1.289 \mathrm{E}+00\end{array}$ $\begin{array}{lllllllllll}.87 & 1.335 \mathrm{E}+00 & 1.282 \mathrm{E}+00 & 1.231 \mathrm{E}+00 & 1.192 \mathrm{E}+00 & 1.171 \mathrm{E}+00 & 1.171 \mathrm{E}+00 & 1.192 \mathrm{E}+00 & 1.231 \mathrm{E}+00 & 1.282 \mathrm{E}+00 \quad 1.335 \mathrm{E}+00 \quad 1.314 \mathrm{E}+00\end{array}$ $\begin{array}{lllllllllll}.93 & 1.322 \mathrm{E}+00 & 1.281 \mathrm{E}+00 & 1.237 \mathrm{E}+00 & 1.204 \mathrm{E}+00 & 1.186 \mathrm{E}+00 & 1.186 \mathrm{E}+00 & 1.204 \mathrm{E}+00 & 1.237 \mathrm{E}+00 & 1.281 \mathrm{E}+00 \quad 1.322 \mathrm{E}+00 \quad 1.330 \mathrm{E}+00\end{array}$

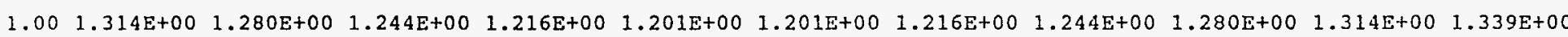

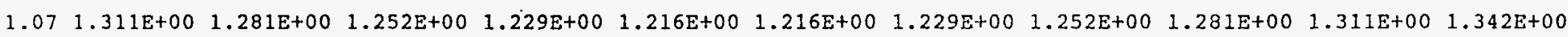

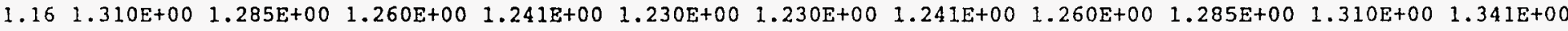

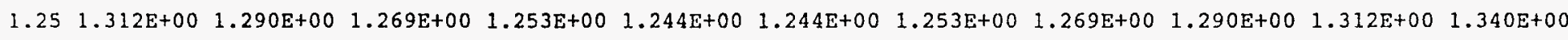

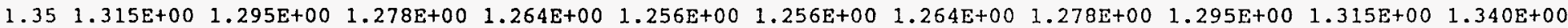
$\begin{array}{lllllllll}1.46 & 1.319 \mathrm{E}+00 & 1.302 \mathrm{E}+00 & 1.287 \mathrm{E}+00 & 1.275 \mathrm{E}+00 & 1.268 \mathrm{E}+00 & 1.268 \mathrm{E}+00 & 1.275 \mathrm{E}+00 & 1.287 \mathrm{E}+00 \quad 1.302 \mathrm{E}+00 \quad 1.319 \mathrm{E}+00 \quad 1.342 \mathrm{E}+00\end{array}$ $\begin{array}{lllllllllllll}1.57 & 1.324 \mathrm{E}+00 & 1.308 \mathrm{E}+00 & 1.295 \mathrm{E}+00 & 1.285 \mathrm{E}+00 & 1.278 \mathrm{E}+00 & 1.278 \mathrm{E}+00 & 1.285 \mathrm{E}+00 & 1.295 \mathrm{E}+00 & 1.308 \mathrm{E}+00 & 1.324 \mathrm{E}+00 & 1.345 \mathrm{E}+00\end{array}$ $\begin{array}{lllllllllll}1.69 & 1.331 \mathrm{E}+00 & 1.315 \mathrm{E}+00 & 1.302 \mathrm{E}+00 & 1.293 \mathrm{E}+00 & 1.287 \mathrm{E}+00 & 1.287 \mathrm{E}+00 & 1.293 \mathrm{E}+00 & 1.302 \mathrm{E}+00 & 1.315 \mathrm{E}+00 \quad 1.331 \mathrm{E}+00 & 1.349 \mathrm{E}+00\end{array}$ $\begin{array}{llllllllll}1.81 & 1.339 \mathrm{E}+00 & 1.322 \mathrm{E}+00 & 1.310 \mathrm{E}+00 & 1.301 \mathrm{E}+00 & 1.295 \mathrm{E}+00 & 1.295 \mathrm{E}+00 & 1.301 \mathrm{E}+00 & 1.310 \mathrm{E}+00 & 1.322 \mathrm{E}+00 \quad 1.339 \mathrm{E}+00 \quad 1.356 \mathrm{E}+00\end{array}$ 


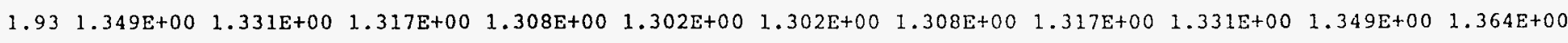

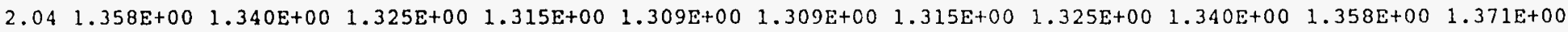

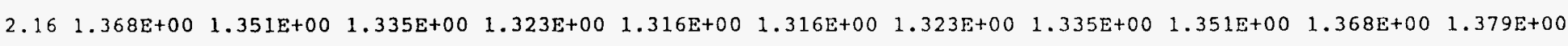
$2.281 .378 \mathrm{E}+00 \quad 1.363 \mathrm{E}+00 \quad 1.346 \mathrm{E}+00 \quad 1.333 \mathrm{E}+00 \quad 1.324 \mathrm{E}+00 \quad 1.324 \mathrm{E}+00 \quad 1.333 \mathrm{E}+00 \quad 1.346 \mathrm{E}+00 \quad 1.363 \mathrm{E}+00 \quad 1.378 \mathrm{E}+00 \quad 1.387 \mathrm{E}+00$ $2.40 \quad 1.387 \mathrm{E}+00 \quad 1.374 \mathrm{E}+00 \quad 1.359 \mathrm{E}+00 \quad 1.345 \mathrm{E}+00 \quad 1.336 \mathrm{E}+00 \quad 1.336 \mathrm{E}+00 \quad 1.345 \mathrm{E}+00 \quad 1.359 \mathrm{E}+00 \quad 1.374 \mathrm{E}+00 \quad 1.387 \mathrm{E}+00 \quad 1.395 \mathrm{E}+00$ $2.521 .396 \mathrm{E}+00 \quad 1.385 \mathrm{E}+00 \quad 1.372 \mathrm{E}+00 \quad 1.360 \mathrm{E}+00 \quad 1.351 \mathrm{E}+00 \quad 1.351 \mathrm{E}+00 \quad 1.360 \mathrm{E}+00 \quad 1.372 \mathrm{E}+00 \quad 1.385 \mathrm{E}+00 \quad 1.3961 \mathrm{E}+00 \quad 1.402 \mathrm{E}+00$ $2.63 \quad 1.404 \mathrm{E}+00 \quad 1.396 \mathrm{E}+00 \quad 1.385 \mathrm{E}+00 \quad 1.374 \mathrm{E}+00 \quad 1.366 \mathrm{E}+00 \quad 1.366 \mathrm{E}+00 \quad 1.374 \mathrm{E}+00 \quad 1.385 \mathrm{E}+00 \quad 1.396 \mathrm{E}+00 \quad 1.404 \mathrm{E}+00 \quad 1.408 \mathrm{E}+00$ $2.75 \quad 1.412 \mathrm{E}+00 \quad 1.405 \mathrm{E}+00 \quad 1.396 \mathrm{E}+00 \quad 1.387 \mathrm{E}+00 \quad 1.380 \mathrm{E}+00 \quad 1.380 \mathrm{E}+00 \quad 1.387 \mathrm{E}+00 \quad 1.396 \mathrm{E}+00 \quad 1.405 \mathrm{E}+00 \quad 1.412 \mathrm{E}+00 \quad 1.414 \mathrm{E}+00$ $2.87 \quad 1.418 \mathrm{E}+00 \quad 1.413 \mathrm{E}+00 \quad 1.406 \mathrm{E}+00 \quad 1.398 \mathrm{E}+00 \quad 1.392 \mathrm{E}+00 \quad 1.392 \mathrm{E}+00 \quad 1.398 \mathrm{E}+00 \quad 1.406 \mathrm{E}+00 \quad 1.413 \mathrm{E}+00 \quad 1.418 \mathrm{E}+00 \quad 1.419 \mathrm{E}+00$ $2.99 \quad 1.424 \mathrm{E}+00 \quad 1.420 \mathrm{E}+00 \quad 1.415 \mathrm{E}+00 \quad 1.408 \mathrm{E}+00 \quad 1.402 \mathrm{E}+00 \quad 1.402 \mathrm{E}+00 \quad 1.408 \mathrm{E}+00 \quad 1.415 \mathrm{E}+00 \quad 1.420 \mathrm{E}+00 \quad 1.424 \mathrm{E}+00 \quad 1.424 \mathrm{E}+00$

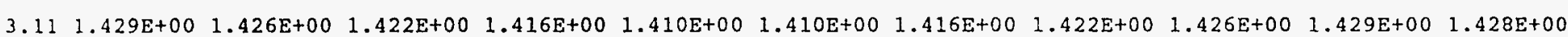

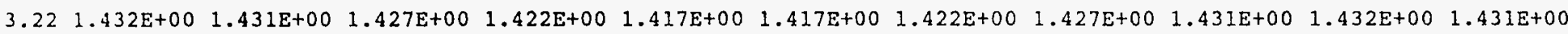

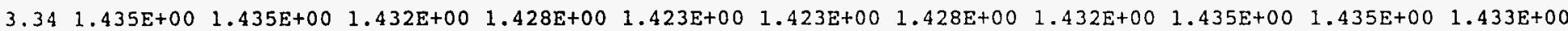

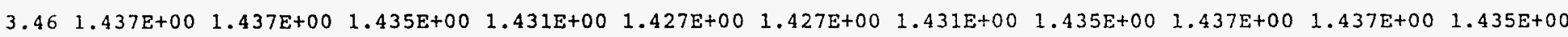

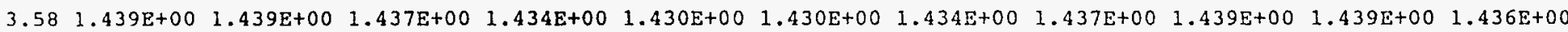

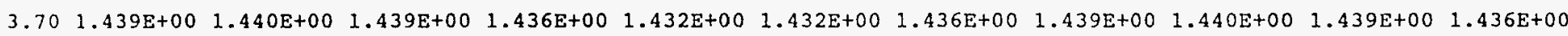

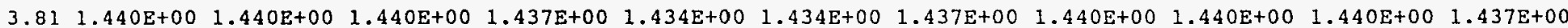

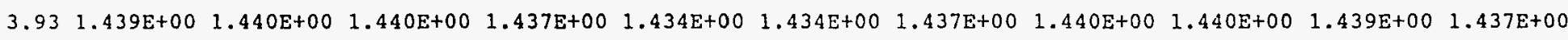

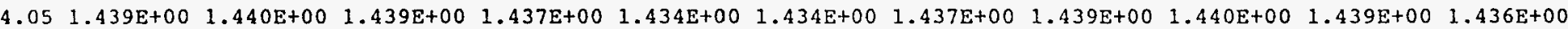
$\begin{array}{lllllllll}4.11 & 1.439 \mathrm{E}+00 & 1.440 \mathrm{E}+00 & 1.439 \mathrm{E}+00 & 1.437 \mathrm{E}+00 & 1.434 \mathrm{E}+00 & 1.434 \mathrm{E}+00 & 1.437 \mathrm{E}+00 \quad 1.439 \mathrm{E}+00 \quad 1.440 \mathrm{E}+00 \quad 1.439 \mathrm{E}+00 \quad 1.436 \mathrm{E}+00\end{array}$ 
$\begin{array}{llllllllllll}.62 & 8.966 \mathrm{E}-01 & 7.604 \mathrm{E}-01 & 6.757 \mathrm{E}-01 & 6.562 \mathrm{E}-01 & 6.674 \mathrm{E}-01 & 6.837 \mathrm{E}-01 & 6.899 \mathrm{E}-01 & 6.058 \mathrm{E}-01 & 4.253 \mathrm{E}-01 \quad 4.143 \mathrm{E}-01\end{array}$ $\begin{array}{llllllllllll}.65 & 9.036 \mathrm{E}-01 & 7.643 \mathrm{E}-01 & 6.781 \mathrm{E}-01 & 6.573 \mathrm{E}-01 & 6.659 \mathrm{E}-01 & 6.843 \mathrm{E}-01 & 7.292 \mathrm{E}-01 & 6.430 \mathrm{E}-01 & 4.202 \mathrm{E}-01 \quad 4.067 \mathrm{E}-01\end{array}$ $.669 .156 \mathrm{E}-01 \quad 7.695 \mathrm{E}-01 \quad 6.803 \mathrm{E}-01 \quad 6.582 \mathrm{E}-01 \quad 6.655 \mathrm{E}-01 \quad 6.859 \mathrm{E}-01 \quad 7.393 \mathrm{E}-01 \quad 7.073 \mathrm{E}-01 \quad 4.106 \mathrm{E}-01 \quad 1.520 \mathrm{E}-01$ $\begin{array}{llllllllllll}.66 & 9.306 \mathrm{E}-01 & 7.754 \mathrm{E}-01 & 6.827 \mathrm{E}-01 & 6.590 \mathrm{E}-01 & 6.653 \mathrm{E}-01 & 6.869 \mathrm{E}-01 & 7.424 \mathrm{E}-01 & 7.596 \mathrm{E}-01 & 5.134 \mathrm{E}-01 & 1.520 \mathrm{E}-01\end{array}$ $\begin{array}{lllllllllllll}.66 & 9.425 E-01 & 7.801 E-01 & 6.847 E-01 & 6.598 E-01 & 6.652 E-01 & 6.882 E-01 & 7.452 E-01 & 7.959 E-01 & 6.559 E-01 & 1.520 E-01\end{array}$ $.679 .560 \mathrm{E}-01 \quad 7.869 \mathrm{E}-01 \quad 6.887 \mathrm{E}-01 \quad 6.618 \mathrm{E}-01 \quad 6.654 \mathrm{E}-01 \quad 6.902 \mathrm{E}-01 \quad 7.492 \mathrm{E}-01 \quad 8.157 \mathrm{E}-01 \quad 8.678 \mathrm{E}-01 \quad 8.710 \mathrm{E}-01$ $\begin{array}{lllllllllll}.68 & 9.649 \mathrm{E}-01 & 7.924 \mathrm{E}-01 & 6.928 \mathrm{E}-01 & 6.641 \mathrm{E}-01 & 6.664 \mathrm{E}-01 & 6.943 \mathrm{E}-01 & 7.558 \mathrm{E}-01 & 8.255 \mathrm{E}-01 & 8.783 \mathrm{E}-01 & 8.815 \mathrm{E}-01\end{array}$ $.699 .786 \mathrm{E}-01 \quad 8.036 \mathrm{E}-01 \quad 7.024 \mathrm{E}-01 \quad 6.705 \mathrm{E}-01 \quad 6.701 \mathrm{E}-01 \quad 7.001 \mathrm{E}-01 \quad 7.635 \mathrm{E}-01 \quad 8.340 \mathrm{E}-018.866 \mathrm{E}-01 \quad 8.898 \mathrm{E}-01$ $.719 .980 \mathrm{E}-01 \quad 8.229 \mathrm{E}-01 \quad 7.203 \mathrm{E}-01 \quad 6.839 \mathrm{E}-01 \quad 6.810 \mathrm{E}-01 \quad 7.127 \mathrm{E}-01 \quad 7.774 \mathrm{E}-01 \quad 8.471 \mathrm{E}-01 \quad 8.989 \mathrm{E}-01 \quad 9.020 \mathrm{E}-01$ $\begin{array}{lllllllllll}.74 & 1.026 \mathrm{E}+00 & 8.532 \mathrm{E}-01 & 7.492 \mathrm{E}-01 & 7.076 \mathrm{E}-01 & 7.026 \mathrm{E}-01 & 7.339 \mathrm{E}-01 & 7.972 \mathrm{E}-01 & 8.631 \mathrm{E}-01 & 9.115 \mathrm{E}-01 & 9.144 \mathrm{E}-01\end{array}$ $\begin{array}{llllllllll}.77 & 1.065 \mathrm{E}+00 & 8.958 \mathrm{E}-01 & 7.903 \mathrm{E}-01 & 7.435 \mathrm{E}-01 & 7.356 \mathrm{E}-01 & 7.642 \mathrm{E}-01 & 8.224 \mathrm{E}-01 & 8.814 \mathrm{E}-01 & 9.245 \mathrm{E}-01 \quad 9.272 \mathrm{E}-01\end{array}$

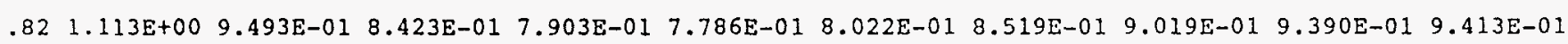
$\begin{array}{lllllllllll}.87 & 1.265 E+00 & 1.010 E+00 & 9.016 E-01 & 8.442 \mathrm{E}-01 & 8.276 \mathrm{E}-01 & 8.453 \mathrm{E}-01 & 8.847 \mathrm{E}-01 & 9.246 \mathrm{E}-01 & 9.553 \mathrm{E}-01 & 9.571 \mathrm{E}-01\end{array}$

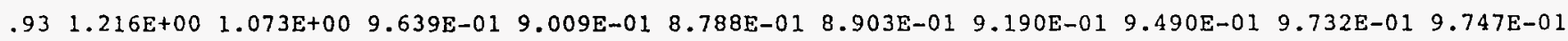

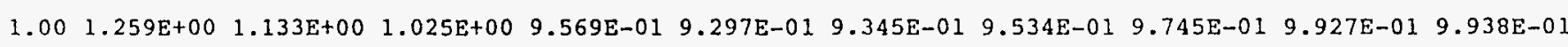
$\begin{array}{lllllllll}1.07 & 1.292 \mathrm{E}+00 & 1.187 \mathrm{E}+00 & 1.083 \mathrm{E}+00 & 1.011 \mathrm{E}+00 & 9.793 \mathrm{E}-01 & 9.772 \mathrm{E}-01 & 9.876 \mathrm{E}-01 & 1.001 \mathrm{E}+00 \quad 1.014 \mathrm{E}+00 \quad 1.014 \mathrm{E}+00\end{array}$ $\begin{array}{llllllllll}1.16 & 1.313 \mathrm{E}+00 & 1.230 \mathrm{E}+00 & 1.134 \mathrm{E}+00 & 1.063 \mathrm{E}+00 & 1.027 \mathrm{E}+00 & 1.018 \mathrm{E}+00 & 1.021 \mathrm{E}+00 & 1.028 \mathrm{E}+00 \quad 1.036 \mathrm{E}+00 \quad 1.037 \mathrm{E}+00\end{array}$ $\begin{array}{llllllllll}1.25 & 1.327 \mathrm{E}+00 & 1.263 \mathrm{E}+00 & 1.180 \mathrm{E}+00 & 1.112 \mathrm{E}+00 & 1.074 \mathrm{E}+00 & 1.059 \mathrm{E}+00 & 1.055 \mathrm{E}+00 & 1.057 \mathrm{E}+00 & 1.060 \mathrm{E}+00 \quad 1.060 \mathrm{E}+00\end{array}$

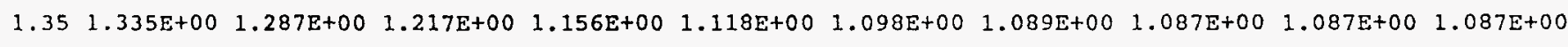

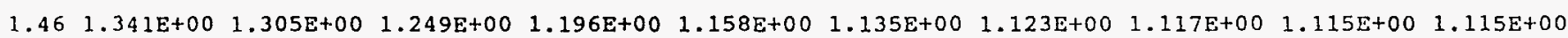
$\begin{array}{llllllllll}1.57 & 1.346 \mathrm{E}+00 & 1.319 \mathrm{E}+00 & 1.274 \mathrm{E}+00 & 1.229 \mathrm{E}+00 & 1.194 \mathrm{E}+00 & 1.170 \mathrm{E}+00 & 1.155 \mathrm{E}+00 & 1.146 \mathrm{E}+00 & 1.141 \mathrm{E}+00 \quad 1.141 \mathrm{E}+00\end{array}$ $\begin{array}{llllllllll}1.69 & 1.352 \mathrm{E}+00 & 1.330 \mathrm{E}+00 & 1.294 \mathrm{E}+00 & 1.256 \mathrm{E}+00 & 1.224 \mathrm{E}+00 & 1.200 \mathrm{E}+00 & 1.183 \mathrm{E}+00 & 1.172 \mathrm{E}+00 & 1.166 \mathrm{E}+00 \quad 1.165 \mathrm{E}+00\end{array}$

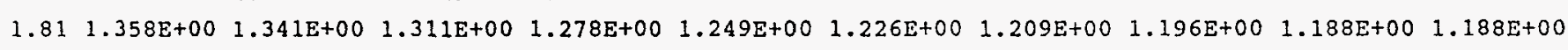
$\begin{array}{llllllllll}1.93 & 1.366 \mathrm{E}+00 & 1.351 \mathrm{E}+00 & 1.326 \mathrm{E}+00 & 1.297 \mathrm{E}+00 & 1.271 \mathrm{E}+00 & 1.249 \mathrm{E}+00 & 1.231 \mathrm{E}+00 & 1.218 \mathrm{E}+00 & 1.209 \mathrm{E}+00 \quad 1.209 \mathrm{E}+00\end{array}$ $\begin{array}{lllllllllll}2.04 & 1.373 E+00 & 1.360 E+00 & 1.339 E+00 & 1.314 E+00 & 1.290 E+00 & 1.269 E+00 & 1.252 E+00 & 1.238 E+00 & 1.229 E+00 & 1.228 E+00\end{array}$ $\begin{array}{lllllllllll}2.16 & 1.380 \mathrm{E}+00 & 1.369 \mathrm{E}+00 & 1.350 \mathrm{E}+00 & 1.328 \mathrm{E}+00 & 1.306 \mathrm{E}+00 & 1.286 \mathrm{E}+00 & 1.270 \mathrm{E}+00 & 1.257 \mathrm{E}+00 & 1.246 \mathrm{E}+00 & 1.246 \mathrm{E}+00\end{array}$ $\begin{array}{lllllllllll}2.28 & 1.387 E+00 & 1.377 E+00 & 1.360 E+00 & 1.340 E+00 & 1.320 E+00 & 1.302 E+00 & 1.286 E+00 & 1.273 E+00 & 1.262 E+00 & 1.262 E+00\end{array}$ $2.40 \quad 1.394 \mathrm{E}+00 \quad 1.384 \mathrm{E}+00 \quad 1.369 \mathrm{E}+00 \quad 1.351 \mathrm{E}+00 \quad 1.332 \mathrm{E}+00 \quad 1.315 \mathrm{E}+00 \quad 1.300 \mathrm{E}+00 \quad 1.287 \mathrm{E}+00 \quad 1.277 \mathrm{E}+00 \quad 1.276 \mathrm{E}+00$

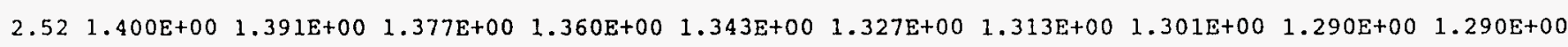
$\begin{array}{lllllllllll}2.63 & 1.406 \mathrm{E}+00 & 1.397 \mathrm{E}+00 & 1.384 \mathrm{E}+00 & 1.369 \mathrm{E}+00 & 1.353 \mathrm{E}+00 & 1.338 \mathrm{E}+00 & 1.324 \mathrm{E}+00 & 1.312 \mathrm{E}+00 & 1.302 \mathrm{E}+00 \quad 1.302 \mathrm{E}+00\end{array}$ $\begin{array}{lllllllll}2.75 & 1.411 E+00 & 1.403 E+00 & 1.390 E+00 & 1.376 E+00 & 1.361 E+00 & 1.347 E+00 & 1.334 E+00 & 1.323 E+00 \quad 1.313 E+00 \quad 1.312 E+00\end{array}$ $\begin{array}{lllllllllll}2.87 & 1.416 \mathrm{E}+00 & 1.407 \mathrm{E}+00 & 1.396 \mathrm{E}+00 & 1.382 \mathrm{E}+00 & 1.368 \mathrm{E}+00 & 1.355 \mathrm{E}+00 & 1.343 \mathrm{E}+00 & 1.332 \mathrm{E}+00 & 1.323 \mathrm{E}+00 & 1.322 \mathrm{E}+00\end{array}$ $\begin{array}{llllllllll}2.99 & 1.420 \mathrm{E}+00 & 1.411 \mathrm{E}+00 & 1.400 \mathrm{E}+00 & 1.388 \mathrm{E}+00 & 1.375 \mathrm{E}+00 & 1.363 \mathrm{E}+00 & 1.351 \mathrm{E}+00 & 1.341 \mathrm{E}+00 & 1.332 \mathrm{E}+00 \quad 1.331 \mathrm{E}+00\end{array}$

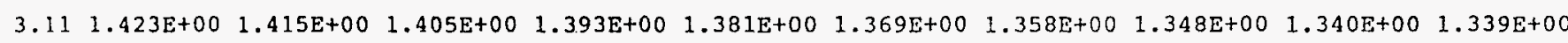
$\begin{array}{lllllllllll}3.22 & 1.426 \mathrm{E}+00 & 1.418 \mathrm{E}+00 & 1.408 \mathrm{E}+00 & 1.397 \mathrm{E}+00 & 1.386 \mathrm{E}+00 & 1.375 \mathrm{E}+00 & 1.365 \mathrm{E}+00 & 1.355 \mathrm{E}+00 & 1.347 \mathrm{E}+00 \quad 1.346 \mathrm{E}+00\end{array}$

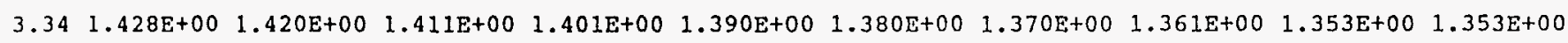


$\begin{array}{llllllllll}3.46 & 1.430 \mathrm{E}+00 & 1.422 \mathrm{E}+00 & 1.414 \mathrm{E}+00 & 1.404 \mathrm{E}+00 & 1.394 \mathrm{E}+00 & 1.384 \mathrm{E}+00 & 1.375 \mathrm{E}+00 & 1.367 \mathrm{E}+00 & 1.359 \mathrm{E}+00 \quad 1.359 \mathrm{E}+00\end{array}$

$\begin{array}{llllllllll}3.58 & 1.431 \mathrm{E}+00 & 1.424 \mathrm{E}+00 & 1.416 \mathrm{E}+00 & 1.407 \mathrm{E}+00 & 1.397 \mathrm{E}+00 & 1.388 \mathrm{E}+00 & 1.380 \mathrm{E}+00 & 1.372 \mathrm{E}+00 & 1.365 \mathrm{E}+00 \quad 1.364 \mathrm{E}+00\end{array}$

$\begin{array}{llllllllll}3.70 & 1.432 \mathrm{E}+00 & 1.425 \mathrm{E}+00 & 1.417 \mathrm{E}+00 & 1.409 \mathrm{E}+00 & 1.400 \mathrm{E}+00 & 1.392 \mathrm{E}+00 & 1.384 \mathrm{E}+00 & 1.376 \mathrm{E}+00 \quad 1.370 \mathrm{E}+00 \quad 1.369 \mathrm{E}+00\end{array}$

$\begin{array}{llllllllll}3.81 & 1.432 \mathrm{E}+00 & 1.426 \mathrm{E}+00 & 1.419 \mathrm{E}+00 & 1.411 \mathrm{E}+00 & 1.403 \mathrm{E}+00 & 1.395 \mathrm{E}+00 & 1.388 \mathrm{E}+00 & 1.381 \mathrm{E}+00 & 1.374 \mathrm{E}+00 \quad 1.374 \mathrm{E}+00\end{array}$

$\begin{array}{lllllllllll}3.93 & 1.432 \mathrm{E}+00 & 1.426 \mathrm{E}+00 & 1.420 \mathrm{E}+00 & 1.412 \mathrm{E}+00 & 1.405 \mathrm{E}+00 & 1.398 \mathrm{E}+00 & 1.391 \mathrm{E}+00 & 1.384 \mathrm{E}+00 & 1.373 \mathrm{E}+00 \quad 1.378 \mathrm{E}+00\end{array}$

$\begin{array}{llllllllll}4.05 & 1.432 \mathrm{E}+00 & 1.427 \mathrm{E}+00 & 1.420 \mathrm{E}+00 & 1.414 \mathrm{E}+00 & 1.407 \mathrm{E}+00 & 1.400 \mathrm{E}+00 & 1.394 \mathrm{E}+00 & 1.387 \mathrm{E}+00 & 1.382 \mathrm{E}+00 \quad 1.381 \mathrm{E}+00\end{array}$

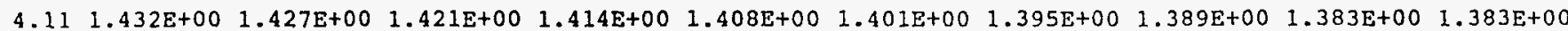

"DENSITY, DNST(I,J)TSTEP O"

"INTERPOLATED CELL CENTER X-VELOCITY/UGO, UGO $=29.30$

"INTERPOLATED CELL CENTER Y-VELOCITY/UGO, UGO= 29.30

"GAS FUEL CONCENTRATION ( $\mathrm{kg}$ fuel/kg mix) TSTEP 0 "

"OXYGEN CONCENTRATION ( $\mathrm{kg}$ oxidizer/kg mix) TIME STEP O"

"PRODUCT CONCENTRATION ( $\mathrm{kg}$ product/kg mix) TSTEP o"

"INERT GAS CONCENTRATION (kg inert/kg mix) TSTEP O"

"SEED VAPOR CONCENTRATION ( $\mathrm{kg}$ seed vapor/kg mix) TSTEP 0 "

"TURB. VISC., TMU $(I, J)$, TSTEP O"

"TURBULENT ENERGY GF(I,J,IK) TIME STEP 0 " 


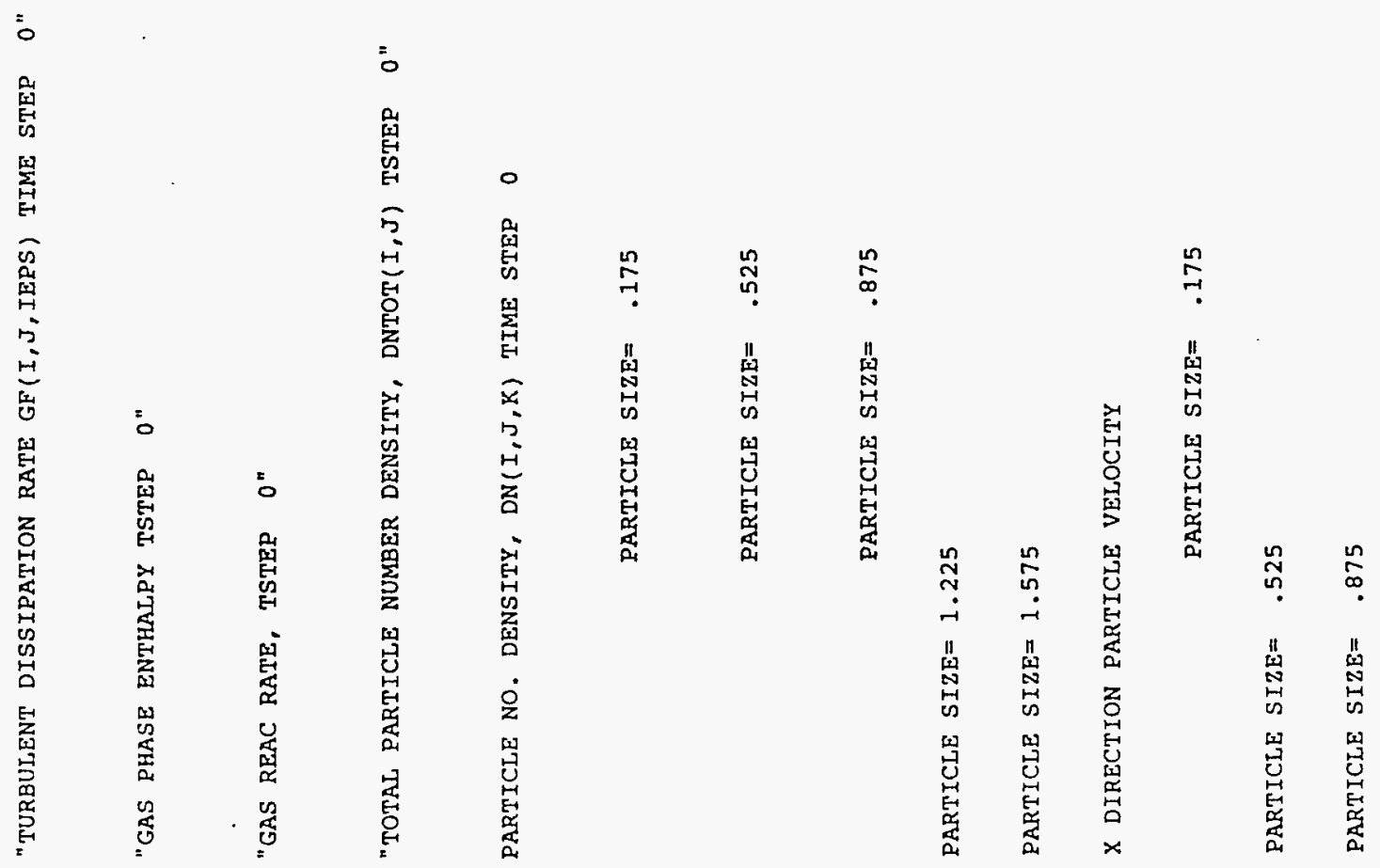


PARTICLE SIZE $=1.225$

PARTICLE SIZE $=1.575$

R DIRECTION PARTICLE VELOCITY

PARTICLE SIZE $=.175$

PARTICLE SIZE $=\quad .525$

PARTICLE SIZE $=\quad .875$

PARTICLE SIZE $=1.225$

PARTICLE SIZE $=1.575$

"PARTICle TEMPERATURE ( TB= .8080) TSTEP $0 "$

PARTICLE SIZE $=\quad .175$

PARTICLE SIZE $=\quad .525$

PARTICLE SIZE $=\quad .875$

PARTICLE SIZE $=1.225$

PARTICLE SIZE $=1.575$

"VOL FRACTION OF PARTICLES"

"EVAP RATE <O=DROP BURN, TSTEP $0 "$ 


\title{
DISTRIBUTION FOR ANL/MHD-97/01
}

\section{Internal}

D.K. Schmalzer

\author{
S.A. Lottes
}

W. Swift

L. Welko (3)

M. Petrick (2)

PRS Files

S.L. Chang

\section{External}

U.S. Department of Energy Office of Scientific and Technical Information (2) Manager, U.S. Department of Energy Chicago Field Office

ANL-E Libraries (2)

ANL-W Library

R.J. Wright - DOE

H. Chambers - DOE

R. Cook - Mississippi State Univ.

J.W. Muehlhauser - Univ. of Tennessee Space Institute

V. Hruby - Busek Co., Inc.

Y.C.L. Wu - Engineering Research Co., Inc.

W.R. Owens - Parsons Power Group

R. Kessler - Textron Systems Div.

J.M. Bauer - TRW, Inc. 


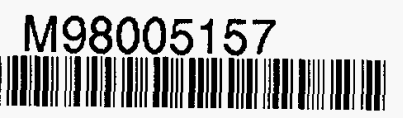

Report Number (14) ANL/MHD_- $97 / 01$

Publ. Date (11) $19971 /$

Sponsor Code (18) DOE/FE, XF
UC Category (19) UC-162, DOEJER

19980622050

DTIC QUALITY INOPECTED I 